\title{
SMART GLOVE AND HAND GESTURE-BASED CONTROL INTERFACE FOR MULTI-ROTOR AERIAL VEHICLES
}

\author{
KIANOUSH HARATIANNEJADI \\ A THESIS \\ IN \\ THE DEPARTMENT \\ $\mathrm{OF}$ \\ ELECTRICAL AND COMPUTER ENGINEERING
}

PRESENTED IN PARTIAL FULFILLMENT OF THE REQUIREMENTS

FOR THE DEGREE OF MASTER OF APPLIED SCIENCE (ELECTRICAL AND COMPUTER ENGINEERING)

AT

CONCORDIA UNIVERSITY

MONTRÉAL, QUÉBEC, CANADA

DECEMBER 2020

(C)KIANOUSH HARATIANNEJADI, 2020 


\title{
CONCORDIA UNIVERSITY
}

\author{
School of Graduate Studies
}

This is to certify that the thesis prepared

\section{By: $\quad$ Kianoush Haratiannejadi \\ Entitled: $\quad$ Smart Glove and Hand Gesture-Based Control Interface for Multi-Rotor Aerial Vehicles}

and submitted in partial fulfillment of the requirements for the degree of

\section{Master of Applied Science (Electrical and Computer Engineering)}

complies with the regulations of this University and meets the accepted standards with respect to originality and quality.

Signed by the final examining committee:

\begin{tabular}{ll}
\hline Dr. Wei-Ping Zhu & Chair \\
\hline Dr. Walter Lucia (CIISE) & External Examiner \\
\hline Dr. Wei-Ping Zhu & Internal Examiner \\
& \\
\hline Dr. Rastko R. Selmic & Supervisor
\end{tabular}

Approved by

Dr. Yousef R. Shayan, Chair

Department of Electrical and Computer Engineering

December 11, 2020

Dr. Mourad Debbabi, Interim Dean

Gina Cody School of Engineering and

Computer Science 


\title{
Abstract
}

\section{Smart Glove and Hand Gesture-Based Control Interface for Multi-Rotor Aerial Vehicles}

\author{
Kianoush Haratiannejadi
}

This thesis introduces two types of adaptable human-computer interaction methods in single-subject and multi-subject unsupervised environments.

In a single-subject environment, a single shot multi-box detector (SSD) is used to detect the hand's regions of interest (RoI) in the input frame. The RoI detected by the SSD model is fed to a convolutional neural network (CNN) to classify the right-hand gesture. In a crowded environment, a region-based convolutional neural network (R-CNN) detects subjects in the frame and the RoI of their faces. These regions of interest are then fed to a facial recognition module to find the main subject in the frame. After the main subject is found, the R-CNN model feeds the right-hand RoI of the main subject to the CNN to classify the right-hand gesture. In both single-subject and multi-subject environments, a fixed number of gestures is used to describe specific commands from the right-hand gestures to the vehicle (take off, land, hover, etc.).

A smart glove on the left hand is used for more precise vehicular control. A motionprocessing unit (MPU) and a set of four flex sensors are used in the smart glove to produce discrete and continuous signals based on the bending value of each finger and the roll angle of the left hand. The generated discrete signals with the flex sensors are fed to a support vector machine (SVM) to classify the left-hand gesture, and the generated continuous signals with the flex sensors and the MPU module are used to determine some characteristics of the gesture command such as throttle and angle of the vehicle. 
Three simultaneous validation layers have been implemented, including (1) HumanBased Validation, where the main subject can reject the robot's behavior by sending a command through the smart glove, (2) Classification Validation, where the main subject can retrain the classifier classes at will, and (3) System Validation, which is responsible for finding the error's source when the main subject performs a specific command through the smart glove.

The proposed algorithm is applied to groups consisting of one, two, three, and four subjects including the main subject to validate and investigate the behavior of the algorithm in various desirable and undesirable situations. The proposed algorithm is implemented on an Nvidia Jetson AGX Xavier GPU. 


\section{Acknowledgments}

I would like to thank my supervisor Prof. Rastko R. Selmic, for his dedicated support and guidance. Prof. Selmic continuously provided encouragement and was always willing and enthusiastic to assist in any way he could throughout the research project.

I cannot forget to thank my family for all the unconditional support in these very intense academic years and send me endless messages of encouragement throughout the process.

I would also like to thank my great friend Mr. Kiarash Ariyankia for always being there for me and support me in any circumstances. Finally, many thanks to all participants that took part in the study and enabled this research to be possible. 


\section{Contents}

List of Figures $\quad$ ix

List of Tables $\quad$ xiii

1 Introduction 1

1.1 Reaserch Topic . . . . . . . . . . . . . . . . . . . 1

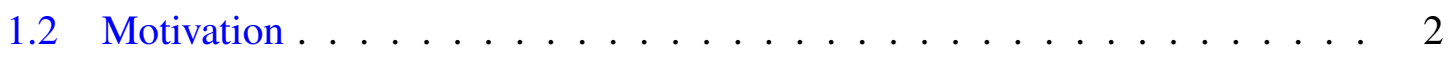

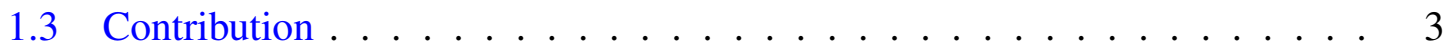

2 Background and Related Works 5

2.1 Introduction . . . . . . . . . . . . . . 5

2.2 Fundamentals ........................ 5

2.2.1 Single Shot Multibox Detector . . . . . . . . . . . 5

2.2.2 Convolutional Neural Network . . . . . . . . . . . . . . . 6

2.2.3 Regional Convolutional Neural Network . . . . . . . . . . . . 11

2.2.4 Support Vector Machine . . . . . . . . . . . . . . . . . 11

2.3 Related Works . . . . . . . . . . . . . . . . . . . 12

3 System Components $\quad 16$

3.1 Introduction . . . . . . . . . . . . . 16

3.2 System Components . . . . . . . . . . . . . . 16

3.2 .1 Arduino . . . . . . . . . . . . . . 16

3.2 .2 Flex Sensors . . . . . . . . . . . . . . . 17 
3.2.3 Arduino Integrated Development Environment _ . . . . . . . . 19

3.2 .4 MPU $6050 \ldots \ldots \ldots \ldots$

3.2.5 Bluetooth Module . . . . . . . . . . . . . . . . . . . . 22

3.2 .6 Webcam . . . . . . . . . . . . . . . . . . 22

$3.2 .7 \quad$ NVIDIA GPU $\ldots \ldots \ldots \ldots \ldots \ldots$

4 System Implementation $\quad 25$

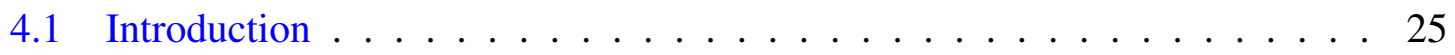

4.2 Smart Glove . . . . . . . . . . . . . . . . . . . . . . 25

$4.2 .1 \quad$ Flex Sensors . . . . . . . . . . . . . . . . . . 25

$4.2 .2 \quad$ MPU $6050 \ldots \ldots \ldots \ldots$

4.3 Hand and Gesture Recognition in a Single-Subject

Environment . . . . . . . . . . . . . . . . . . 33

4.3.1 Hand Detector . . . . . . . . . . . . . . . . . . 34

4.3.2 Gesture Classifier . . . . . . . . . . . . . . . . . . . 35

4.3.3 Retrainer Module . . . . . . . . . . . . . . . . . . 36

4.4 Hand and Gesture Recognition in a Multi-Subject

Environment . . . . . . . . . . . . . . . . . . 37

4.4.1 Skeleton Detection Module . . . . . . . . . . . . . . . . . . 39

4.4.2 Facial Recognition Module . . . . . . . . . . . . . . . . . 40

4.4.3 Right-Hand Detection Module . . . . . . . . . . . . . . . . . 41

4.4.4 Right-Hand Classifier . . . . . . . . . . . . . . . . . . . 42

4.4.5 Retrainer Module . . . . . . . . . . . . . . . . . . . . 42

4.5 Validation Modules . . . . . . . . . . . . . . . . . . . 42

4.5.1 Human-in-the-Loop Validation $\ldots \ldots \ldots$. . . . . . 43

4.5.2 Gesture Recognition Validation . . . . . . . . . . . . . . 44

4.5.3 System Validation . . . . . . . . . . . . . . . 45

5 Validation and Verification $\quad 47$

5.1 Introduction $\ldots \ldots \ldots \ldots \ldots \ldots \ldots \ldots \ldots \ldots \ldots \ldots$ 


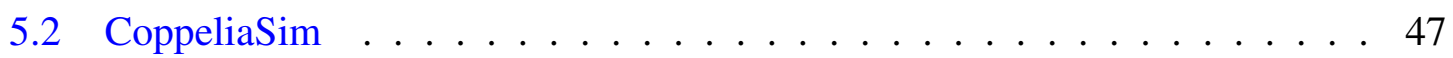

5.3 Gesture Recognition Using Smart Glove . . . . . . . . . . . . . . 48

5.3.1 Gesture Classifier Using the SVM Model . . . . . . . . . . . . 49

5.4 Gesture Recognition in a Single-Subject Environment . . . . . . . . . . 50

5.4.1 Hand Detection Using the SSD Model . . . . . . . . . . . . 50

5.4.2 Gesture Classifier Using the CNN Model . . . . . . . . . . . 52

5.5 Gesture Recognition in a Multi-Subject Environment . . . . . . . . . 53

5.5.1 Gesture Classifier Using the CNN Model . . . . . . . . . . . . 54

5.5.2 Skeleton Detection Using R-CNN Model … . . . . . . . . 55

5.6 Effect of Activating a Flex Sensor on Velocity of the Drone Movement . . . 56

5.7 Test Scenarios . . . . . . . . . . . . . . . . . . . . . . . . . . 57

5.7.1 Scenario 1: System Validation With No Penalty . . . . . . . . 58

5.7.2 Scenario 2: Penalty on the Flight Controller . . . . . . . . . . 59

5.7.3 Scenario 3: Penalty on the Classifier . . . . . . . . . . . . 60

5.7.4 Scenario 4: Frame Contains Both Hands . . . . . . . . . . . . 62

5.7.5 Scenario 5: Frame Without Right Hand . . . . . . . . . . 62

5.7.6 Scenario 6: Frame Contains Right Hand but There Is No Posture . . 62

5.7.7 Scenario 7: Frame Contains Correct Hand _ . . . . . . . . 63

6 Conclusion and Future Work $\quad 76$

6.1 Limitations . . . . . . . . . . . . . . . . 78

6.2 Future Work . . . . . . . . . . . . . . . . . . 78 


\section{List of Figures}

Figure 2.1 Single shot multi-box model architecture. $\ldots \ldots \ldots \ldots$

Figure 2.2 Convolutional neural network model architecture in the gesture recognition model. . . . . . . . . . . . . . . . . . . . . 11

Figure 3.1 Arduino UNO board. . . . . . . . . . . . . . . . 17

Figure 3.2 Flex sensor with two pins for producing voltage on them. . . . . 18

Figure 3.3 Flex sensors calibration diagram. . . . . . . . . . . 18

Figure 3.4 Connection between all flex sensors and Arduino pins. . . . . . . . 19

Figure 3.5 Calibration process: MPU module and the flex sensors. . . . . . . 20

Figure 3.6 Gestures with the smart glove. Bending values for four flex sensors on the glove are shown as F1, F2, F3, and F4 for four fingers of the left hand. 21

Figure 3.7 MPU $6050 \ldots \ldots \ldots \ldots \ldots \ldots \ldots \ldots$

Figure 3.8 Arduino UNO Bluetooth module. . . . . . . . . . . . . . . . 22

Figure 3.9 Smart glove. . . . . . . . . . . . . . . . . . 23

Figure 3.10 Cameras used in the project. . . . . . . . . . . 23

Figure 3.11 The main processor on the robot, the NVIDIA GPU. . . . . . . . . 24

Figure 4.1 Smart Glove designed in this project. . . . . . . . . . . . 26

Figure 4.2 Finger's bending values of four flex sensors. As indicated on the horizontal axis, a combination of four bending values from the flex sensors

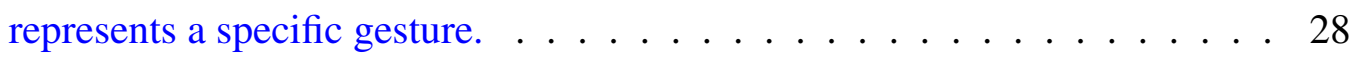

Figure 4.3 MPU response in three zones by fluctuating the smart glove. . . . 30 
Figure 4.4 Gesture recognition module with the smart glove. This unit by using flex sensors calculates the bending value for each finger and then an SVM classifier classifies the gesture based on those bending value. An MPU module is used to calculate the angle of the left hand. . . . . . . . . . . . 30

Figure 4.5 Smart glove gestures with the GUI. . . . . . . . . . . . . . . 31

Figure 4.6 Smart glove gestures and their flex sensors outputs. . . . . . . . . 32

Figure 4.7 System block diagram for a single-subject environment, including three separate validation modules: (i) Classification Validation, (ii) System Validation, and (iii) human-in-the-loop validation. . . . . . . . . . . . 33

Figure 4.8 Hand Detection and Classification Unit block diagram in a singlesubject environment. . . . . . . . . . . . . . . . 35

Figure 4.9 Four specified right-hand gestures as the webcam inputs, for classification. Each gesture represents a particular command that is given to the multi-rotor aerial vehicle as a control signal. The possible classes are (a) Palm (Class \#1): take off, (b) Fist (Class \#2): land, (c) Victory sign (Class \#3): turn left, and (d) OK sign (Class \#4): turn right. . . . . . . . . . . . 36

Figure 4.10 System block diagram for a multi-subject environment including three separate validation modules: (i) Classification Validation, (ii) System Validation, and (iii) human-in-the-loop validation. . . . . . . . . . . . 38

Figure 4.11 Flowchart of hand gesture recognition in a multi-subject environment. 38

Figure 4.12 Gesture Classification block diagram in a multi-subject environment. 39

Figure 4.13 Numbered joints for the skeleton algorithm. The initial keypoint is the nose. . . . . . . . . . . . . . . . . . . 40

Figure 4.14 Face landmark detection. . . . . . . . . . . . . . . . . 41

Figure 5.1 Multi-rotor aerial vehicle control using hand gestures and smart glove with self-validation. . . . . . . . . . . . . . . 48

Figure 5.2 Support vector machine model confusion matrix for the left-hand gesture recognition. . . . . . . . . . . . . . 
Figure 5.3 Flex sensors bending values through the 16 gestures of the smart glove. The generated signals with the flex sensors are used for left-hand gesture recognition and the generation of continuous control signals. As indicated on the horizontal axis, a combination of four bending values from the flex sensors represents a specific gesture . . . . . . . . . . . . 50

Figure 5.4 Total loss of the training process with respect to the steps. . . . . . . 51

Figure 5.5 Training set versus validation set in a single-subject environment:

(a) Accuracy; (b) Loss. . . . . . . . . . . . . . . . . . . . . . . 53

Figure 5.6 Training set versus validation set in a multi-subject environment:

(a) Accuracy; (b) Loss. . . . . . . . . . . . . . . . . . . . . 55

Figure 5.7 Activating the third flex sensor by bending the third flex sensor $\left(f_{3}\right)$ and its effect on the velocity of the drone. . . . . . . . . . . . 57

Figure 5.8 Three cases for the System Validation: 1) No penalty, 2) Penalty on the Flight Controller, and 3) Penalty on the classifier. The commands are: Class \#1: take off, Class \#2: land, Class \#3: turn left, and Class \#4: turn

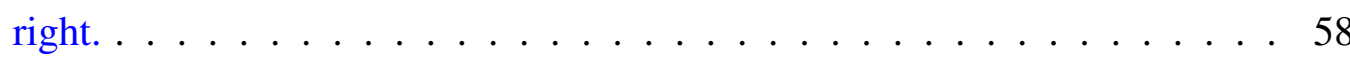

Figure 5.9 System without penalty. The commands are: Class \#1: take off, Class \#2: land, Class \#3: turn left, and Class \#4: turn right. . . . . . . . . 59

Figure 5.10 Penalty signal for the controller. The commands are: Class \#1: take off, Class \#2: land, Class \#3: turn left, and Class \#4: turn right. . . . . . . 59

Figure 5.11 Penalty signal for the classifier. The commands are: Class \#1: take off, Class \#2: land, Class \#3: turn left, and Class \#4: turn right. . . . . . . 60

Figure 5.12 Gesture recognition on a single subject gesturing in front of the camera (series 1). The commands are: Class \#1: take off, Class \#2: land, Class \#3: turn left, and Class \#4: turn right. . . . . . . . . . . . . . . . 64

Figure 5.13 Gesture recognition on a single subject gesturing in front of the camera (series 2). The commands are: Class \#1: take off, Class \#2: land, Class \#3: turn left, and Class \#4: turn right. 
Figure 5.14 Gesture recognition on two (2) subjects including a main subject gesturing in front of the camera $($ series 1$) \ldots \ldots 66$

Figure 5.15 Gesture recognition on two (2) subjects including a main subject gesturing in front of the camera $($ series 2$) \ldots \ldots$. . . . . . . . 67

Figure 5.16 Gesture recognition on two (2) subjects including a main subject gesturing in front of the camera $($ series 3$) \ldots \ldots$. . . . . . . . 68

Figure 5.17 Gesture recognition on two (2) subjects including a main subject gesturing in front of the camera (series 4) . . . . . . . . . . . . . 69

Figure 5.18 Gesture recognition on two (2) subjects including a main subject gesturing in front of the camera (series 5). . . . . . . . . . 70

Figure 5.19 Gesture recognition on three (3) subjects including a main subject gesturing in front of the camera $($ series 1$) \ldots \ldots . \ldots 71$

Figure 5.20 Gesture recognition on three (3) subjects including a main subject gesturing in front of the camera (series 2) . . . . . . . . . . 72

Figure 5.21 Gesture recognition on three (3) subjects including a main subject gesturing in front of the camera $($ series 3$) \ldots \ldots . \ldots 73$

Figure 5.22 Gesture recognition on group of four (4) subjects including a main subject performing in front of the camera (series 1) . . . . . . . . . . 74

Figure 5.23 Gesture recognition on group of four (4) subjects including a main subject performing in front of the camera (series 2) . . . . . . . . 75 


\section{List of Tables}

Table 2.1 Number of layers used to design the CNN model in a single-subject

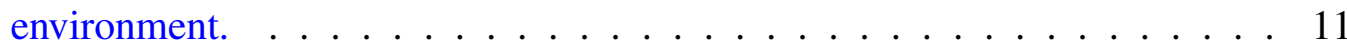

Table 2.2 Comparison between the proposed method and similar approaches. - 15

Table 4.1 Gestures for the smart glove and their corresponding functions. . . . 29

Table 4.2 Flex sensors features and specifications. . . . . . . . . . . . . . . . 29

Table 4.3 Confusion matrix for the CNN model. Class \#1: take off, Class \#2:

land, Class \#3: turn left, and Class \#4: turn right. . . . . . . . . . . . . . 44

Table 4.4 Configuration of the proposed CNN layers. . . . . . . . . . . . . 46

Table 5.1 Righ-hand gesture classifier training and validation results for different data sizes. . . . . . . . . . . . . . . . . . 53

Table 5.2 Gesture recognition in a multi-subject environment: Experimental results for four different group populations. A group of $m$ subjects refers to the population of subjects in the input frame which means there are $m$ subjects in the frame. . . . . . . . . . . . . . 56

Table 5.3 Effect of the activated flex sensor on the drone velocity. Note: $f_{n}$ : bending value of the $n$-th flex sensor, $G \# m$ : $m$-th gesture with the smart glove, $V_{G}(t)$ : drone velocity. . . . . . . . . . . . . . . . 57

Table 5.4 Penalty on the classifier: Class \#4. Classification Validation decides to retrain the model on Class \#4 in three steps to improves the confidence from $54.55 \%$ to $79.19 \%$. The four classes are: Class \#1: take off, Class \#2: land, Class \#3: turn left, and Class \#4: turn right. . . . . . . . . . . . 60 
Table 5.5 Penalty on the classifier: Class \#2. Classification Validation decides to retrain the model on Class \#2 in three steps to improves the confidence from $16.67 \%$ to $100 \%$. The four classes are: Class \#1: take off, Class \#2: land, Class \#3: turn left, and Class \#4: turn right. . . . . . . . . . . 61

Table 5.6 Mapping table between classifier output and the algorithm output. . . 63 


\section{Chapter 1}

\section{Introduction}

\subsection{Reaserch Topic}

This thesis proposes a method for capturing and classifying the right- and left-hand gestures of a main subject and transforming them into commands that control a robot such as an unmanned aerial vehicle (UAV). This method uses various neural networks, including a convolutional neural network $(\mathrm{CNN})$, a region-based convolutional neural network (R$\mathrm{CNN}$ ), a single shot multi-box detector (SSD), and a support vector machine (SVM). A new self-validating interface was developed for controlling multi-rotor aerial vehicles in single-subject and multi-subject environments. In recent years, multi-rotor aerial vehicles have used in various applications such as monitoring, mapping, person-following [1] and transportation [2].

For a single-subject environment, the proposed method uses right-hand gesture recognition, where a hand in each image frame is detected using an SSD, and a CNN model then performs a classification. For a multi-subject environment, the proposed method first uses an R-CNN to detect subjects in the input frame, and a facial recognition module to find the subject of interest. Then, the R-CNN model detects the main subject's right-hand region, and a CNN model classifies the gestures of the detected right hand into one of the predefined classes. The proposed method differs in the process according to whether the environment is single- or multi-subject. 
Some aspects of the proposed method in this thesis are the same for both environments. In single- or multi-subject environments, the left-hand controls the aerial vehicle, which occurs simultaneously with the right-hand control, uses a smart glove with four flex sensors and a motion-processing unit (MPU). These sensors feed data into an SVM for left-hand gesture recognition. An advantage of this combined interface is that wearable devices, such as smart gloves, do not depend on environmental conditions such as lighting or the user's physical features. The gesture recognition interface should be sufficiently robust and adaptable to be able to handle various hand shapes and sizes.

\subsection{Motivation}

Gesture recognition system recognizes human gestures and classifies them such that they can be used as control commands. In modern gesture recognition systems, human gestures are transmitted via special gadgets (such as gloves) equipped with various sensors or read by a camera. The captured data are then interpreted as commands and used as inputs to control applications or robotic devices.

Currently, various techniques involving wearable gadgets and gesture recognition have been used to advance methods of human-robot interaction [3, 4, 5, 6]. Applications of these techniques include a wearable gesture recognition glove that controls robotic devices in disaster management [7] and wearable motion sensors that drive a virtual car [8]. Other examples include robotic devices that maintain a clean environment [9] and wearable motion sensors for the impaired hearing persons [10].

Hand gesture recognition systems can be categorized into glove-based and vision-based systems. A glove-based gesture recognition system consists of a glove and sensors that captures data. The glove extracts the configuration and motion of the user's hand. Authors in [10] translated a sign language gestures into text, where their method consists of a series of accelerometers on each finger of a glove and other sensors on the shoulders that send electrical signals to a microcontroller. The microcontroller then interprets the action associated with the gesture. In addition to the glove-based part of the proposed method where 
enables the system to recognize the hand gestures using wearable sensors, the proposed method also has a vision-based part which uses visual inputs such as images from a camera to extract the features to be used in gesture recognition.

\subsection{Contribution}

We propose an adaptable, closed-loop system that can recognize and classify right-hand and left-hand gestures simultaneously in both single- and multi-subject environments. The flex sensors and the MPU on the smart glove enable the user to produce continuous-time control commands (e.g., the throttle of a drone or its roll angle). We demonstrate how the proposed method classifies gestures and how it handles various undesirable situations.

The proposed approach has the following novelties and advantages in comparison with existing methods in $[3,4,5,6,7,10,11]$ :

(1) The proposed smart glove produces 16 different gestures (commands) including two control signals that are used to control of an unmanned vehicle.

(2) A hand recognition module and background removal method are used before gesture classification to improve classifier performance.

(3) Detection of the main subject's right-hand region and classification of the main subject's right-hand gestures have been implemented.

(4) The system is adaptable to new users due to online learning.

(5) Self-validation of the classifier is proposed which improves the reliability.

(6) System validation and a human-in-the-loop validation increase the overall system reliability.

Furthermore, the smart glove, with 16 different commands is appended to the image processing system (creates four commands) to provide more control commands in comparison to gesture classification alone. For instance, angle variation in turn right and turn left 
commands is possible by changing the flex sensors' bending value on the left hand fingers. Similarly, increasing and decreasing the altitude of the drone is feasible by altering the angle of the smart glove. The control precision of this combined method outperforms the smart glove and image processing techniques on their own. 


\section{Chapter 2}

\section{Background and Related Works}

\subsection{Introduction}

This chapter presents an introduction and an overview of the utilized neural networks such as CNN and R-CNN and classification methods such as SSD and SVM in this thesis. Additionally, recent related works on gesture recognition with cameras and wearable gadgets are discussed and compared with the proposed method.

\subsection{Fundamentals}

In the proposed algorithm, we use a series of neural networks for various purposes. For instance, an SSD model is used to detect a hand in a single-subject environment, and an $\mathrm{R}-\mathrm{CNN}$ model is used to find the subjects in each input frame. A brief introduction to each model is presented in the following subsections.

\subsubsection{Single Shot Multibox Detector}

An SSD is an object detection model with a two-component structure [12]. The model takes an image as an input and categorizes the various objects in this image into different classes. Figure 2.1 shows that for image classification, the model applies a base network, 


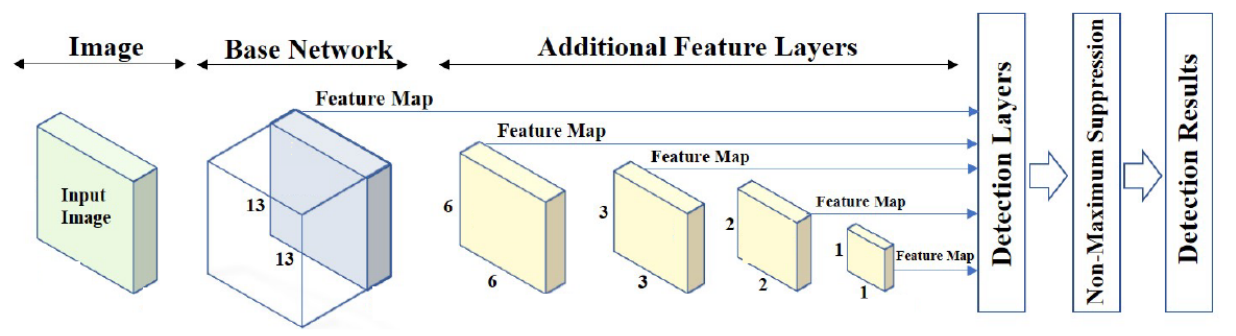

Figure 2.1: Single shot multi-box model architecture.

including a standard architecture, while for object detection, it uses a structure of additional feature layers. This auxiliary structure contains convolutional feature layers sorted by size in descending order, placed at the end of the base network to perform object detection at different scales.

The proposed method in this thesis uses the SSD object detection model for hand tracking in the single-subject scenario [13]. Subsequently, a non-maximum suppression (NMS) algorithm [14] is used to merge all the detected regions related to similar hand gestures. The NMS output is used as an input to the Hand Gesture Classification module. Object detection is performed at different scales and uses boxes at various aspect ratios similar to the faster R-CNN [12]. Since each convolutional layer operates at a different scale, the model can detect objects of various sizes. This model does not require region proposals (based on sets of rectangles) for the convolution layers. Instead, it uses various bounding boxes and then adjusts each bounding box as part of a prediction in a single network, which accelerates calculations.

\subsubsection{Convolutional Neural Network}

The CNN model consists of several layers of artificial neural networks (ANNs) stacked in a cascading structure [15]. For the CNN hidden layers we choose three layers of maxpooling, two layers of dropout, five rectified activation functions in different layers, one sigmoid activation function, one flattening layer, and two fully connected layers.

In the convolutional layer, the $\mathrm{CNN}$ extracts the feature maps from the input image using feature detectors. When feature maps are connected, the convolutional layer is formed. 
Since the convolutional operation is linear, the output of the convolution is passed through an activation function to make the output nonlinear, and a rectified linear unit (ReLU) and a sigmoid function are used as activation functions to enhance the nonlinearity of the feature maps.

\section{Pooling Layer:}

The purpose of pooling layers is to decrease the feature maps' resolution and summarize the input distortions in a smaller feature map. We use max-pooling method in the CNN design to extract the highlights from the input frame. In max-pooling, we select the largest element within each feature map such that [16]:

$$
y_{i, j}=\max _{(p, q) \in R_{i, j}} x_{p, q}
$$

where $x_{p, q}$ is defined as

$$
x_{p, q}=\sum_{i=1}^{m} \omega_{i} x_{p, q_{i}},
$$

in (1), $y_{i, j}$ is the output of the pooling function associated with the feature map region $R_{i, j}$, and $x_{p, q}$ is the input element at location $(p, q)$ which embodies an area around the position $(i, j)$ in the input frame. In (2), $\omega_{i}$ is the $i$-th weight associated with $x_{p, q_{i}}$ which is $i$-th element of $x_{p, q}$, and $m$ is the number of element in input $x_{p, q}$.

By using a max-pooling technique, unnecessary information is removed from the generated feature maps in the convolutional layer and reduces the map's dimensions. The maximum value in each sub-region of the feature maps is stored, and the remaining values are deleted as unnecessary information. The pooled feature maps are generated to form the max-pooling layer.

\section{Dropout:}

The dropout technique is used to prevent overfitting during the training process. The dropout technique is applied only during the training time and at each iteration, which 
temporarily disables a neuron with a probability of $p$. All the inputs and outputs to the disabled neuron are frozen at the current iteration. The dropped-out neurons are tested with probability $p$ at every iteration of training, so a dropped-out neuron at one step can be activated at the next iteration [17].

The dropout-rate $p$ is corresponding to $p \%$ of the neurons being dropped-out during the whole training process. The dropout controls the participation of each neuron $x_{j}$ with an independent Bernoulli random variable $\alpha_{j}$ for each step [18]:

$$
y_{i}=\frac{1}{p} \sum_{j=1}^{N} \omega_{j, i}\left(\alpha_{j} \cdot x_{j}\right), \alpha_{j} \sim B(1, p),
$$

where $B(1, p)$ is the Bernoulli function which generates the independent random variable $\alpha_{j}$ with probability $p$. The scaling factor $\frac{1}{p}$ scales the output $y_{i}$ to keep the expected value of the output during the training process. The weight from input $x_{j}$ to input $x_{i}$ is denoted by $\omega_{j, i}$, and $N$ is the number of neurons connected to the neuron $x_{i}$.

\section{Activation Function:}

Activation functions are used to determine the neural network's output in classification problems. Furthermore, an activation function limits the amplitude of the output of a neuron and maps the output values between 0 to 1 or -1 to 1 depending upon the function's type $[17,19]$.

Comparison in [19] between most commonly used activation functions reveals that the suitable activation functions for training an image processing-based algorithm with the proposed architecture in this thesis are ReLU and sigmoid activation functions. Moreover, the training process requires fewer iterations with ReLU and sigmoid activation function than the other activation functions to solve nonlinear problems. Additionally, we find that our CNN's accuracy is $5 \%$ higher with these two functions than with other activation functions such as radial basis function and hyperbolic tangent function.

ReLU has output 0 if the input is less than 0 , and has the same value as the input if the input is greater than 0 : 


$$
f(x)=\max (x, 0)
$$

where $f(x)$ is the output of the function and $x$ is the input neuron.

Sigmoid functions are used in machine learning for logistic regression and neural network implementations. Sigmoid function and its derivative reduces the time required for training and building the model [19]. The sigmoid activation function is mostly used in neural networks trained to classify the input into classes.

We use the sigmoid function as it transforms the neuron's output $g\left(x_{i}\right)$ to values between 0 and 1 , and is defined as:

$$
g\left(x_{i}\right)=\frac{1}{1+e^{-x_{i}}}
$$

where input neuron $x_{i}$ is defined as:

$$
x_{i}=\sum_{j=1}^{N} \omega_{j} x_{i j},
$$

and $\omega_{j}$ denotes the weight associated to the $j$-th element of input $x_{i}$ and $N$ is the number of element in input $x_{i}$. Here, we apply the sigmoid function to our CNN design to obtain each hand-gesture class's probability.

\section{Optimizer:}

The main purpose of using optimizer is to reduce the losses, increase the learning rate, and provide the most accurate results [20]. Comparisons [20] and [21] between adaptive moment estimation (Adam), gradient descent, adagrad, and adadelta show that the suitable optimizer based on the proposed neural network structure is Adam optimizer because of its algorithm speed, computation capacity and obtained results with the proposed algorithm. The weight update law for Adam optimizer [22] is given by 


$$
\omega_{t+1}=\omega_{t}-\frac{\eta \cdot \hat{m}_{t}}{\sqrt{\hat{v}_{t}+\varepsilon}}
$$

where $\omega_{t}$ and $\omega_{t+1}$ are the current model weight and the next iteration model weight, respectively. The learning rate (step size) in Adam equation is denoted by $\eta$, and to avoid division by zero, a small positive term $\varepsilon$ is added in the denominator. The bias correction of the moving averages $\widehat{m}_{t}$ and $\widehat{v}_{t}$ in the Adam optimizer are as follows:

$$
\begin{aligned}
\hat{m}_{t} & =\frac{m_{t}}{1-\beta_{1}^{t}}, \\
\hat{v}_{t} & =\frac{v_{t}}{1-\beta_{2}^{t}},
\end{aligned}
$$

where $m_{t}$ and $v_{t}$ are moving averages as follows:

$$
\begin{gathered}
m_{t}=\left(1-\beta_{1}\right) g_{t}^{2}+\beta_{1} m_{t-1}, \\
v_{t}=\left(1-\beta_{2}\right) g_{t}^{2}+\beta_{2} v_{t-1},
\end{gathered}
$$

and $g_{t}$ is gradient on current mini-batch, and $\beta_{1}$ and $\beta_{2}$ are the exponential decay rates.

\section{Flattening Layer:}

The flattening layer converts two-dimensional max-pooling outputs to a one-dimensional array. Finally, the fully connected layer is created by connecting each neuron to all neurons in the previous layer to classify the extracted features.

The number of layers in the CNN model in the proposed method is shown in Table 2.1, and the CNN model structure is illustrated in Figure 2.2. 


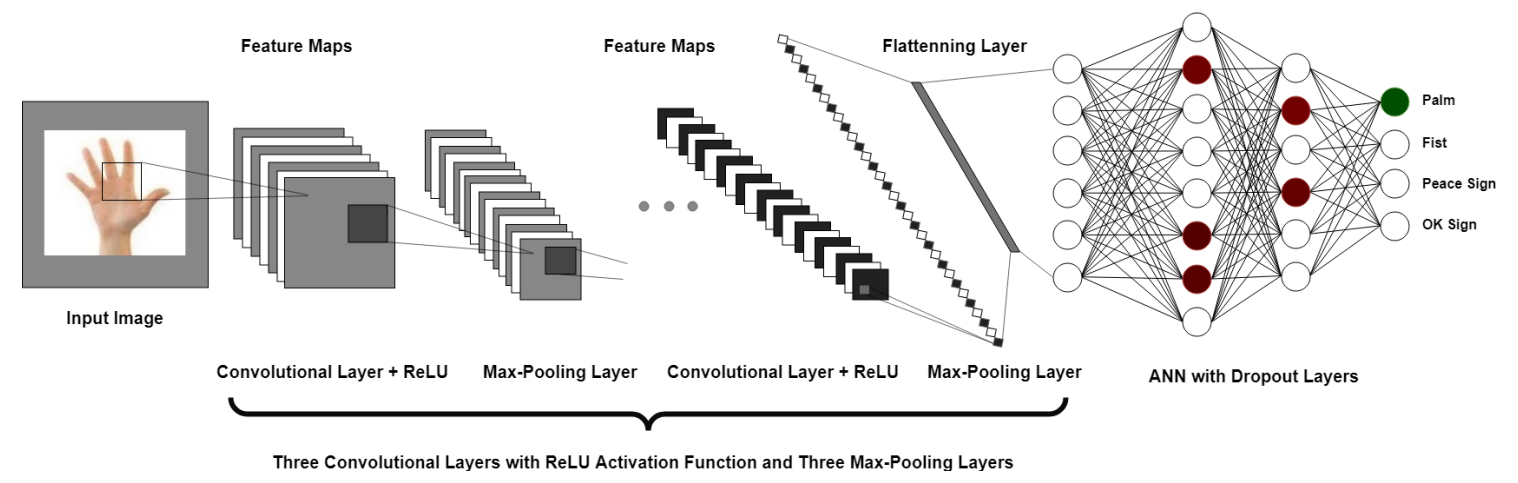

Figure 2.2: Convolutional neural network model architecture in the gesture recognition model.

Table 2.1: Number of layers used to design the CNN model in a single-subject environment [13].

\begin{tabular}{ccc}
\hline Layers Level & Layers & Number of Layers \\
\hline 1 & Convolutional Layer & 3 \\
2 & Max-pooling Layer & 3 \\
3 & Flattening Layer & 1 \\
4 & Fully Connected Layer & 2 \\
5 & Rectifier Activation Function & 5 \\
6 & Dropout Layer & 2 \\
7 & Sigmoid Activation Function & 1 \\
\hline
\end{tabular}

\subsubsection{Regional Convolutional Neural Network}

An R-CNN, consists of two steps [23]. In the first step, it uses selective search, which extracts nearly 2,000 region suggestions from the input image into boxes. Hence, instead of attempting to classify a very large number of regions, the network can work with only 2,000 regions. In the second step, it extracts all the features from those 2,000 regions independently using a large CNN for classification. The R-CNN classifies each region by feeding data from the last layer into SVMs for each suggested box in the input image.

\subsubsection{Support Vector Machine}

Support vector machine (SVM) is an universal learning machine, which is applied to both regression [24] and pattern recognition [25]. An SVM uses a kernel to map the data 
from input space to a high-dimensional feature space in which the problem becomes linearly separable [26]. The decision function of an SVM is related not only to the number of support vectors and their weights but also to the a priorly chosen kernel that is called the support vector kernel [24]. There are three advantages of using SVM model in the classification problems: (i) conversion of the classification problem from a complex classification problem to the computation of a linear decision function, (ii) absence of local minimum in the SVM optimization problem, and (iii) a sparse solution which causes a computationally efficient decision function.

SVM uses Cover's theorem, which guarantees that any data set becomes arbitrarily separable as the data dimension grows, and a class of functions called kernels is used in SVM to achieve this goal. Many kinds of kernels can be used, such as the gaussian kernel or gaussian radial basis function (RBF) when there is no prior knowledge about the data, polynomial kernel for image processing, ANOVA radial basis kernel in regression problems, and linear kernel when dealing with large sparse data vectors. In the proposed method, the smart glove's gathered data has four dimensions (four flex sensors), and there is a clear margin of separation between classes. Since the SVM model works relatively well in these two conditions, we choose the SVM as the left-hand classifier.

Since the proposed algorithm is designed to control a drone in a real-time scenario, we need a kernel in the SVM model that is fast and has a real-time response time of less than a second to generate the output and send it to the next module. Response time and training an SVM with a dataset with four dimensions with a linear kernel are faster than any other kernel. In the next chapters in this thesis, we will use the linear kernel to classify the smart glove data into pre-defined hand gestures.

\subsection{Related Works}

Human-robot interaction has broad applications such as gaming [27] and controlling and navigation of a quadrotor in GPS-denied environments [28]. In several previous approaches, $[8,28,29,30]$, the user must stand alone and show only the requested hand. 
Moreover, the use of only right-hand gestures to interact with a drone is presented in [31]. In contrast, the proposed algorithm can distinguish and receive commands from the gestures of both hands simultaneously, and the proposed system can interact with and recognize the main subject in a multi-subject environment.

An effective human-robot interaction requires a gesture vocabulary [31, 32]. While [32] used only right-hand gestures to interact with and command waiters based on six gestures, the proposed method in this thesis uses an image processing and self-validation technique with four gestures and a smart glove with 16 distinct gestures.

Authors in [33] proposed a gesture recognition method using depth information from pixels for the human skeleton where depth information is used to segment the body into joints. In this thesis, we use the Skeleton Detection module on 2D images to determine all the users presented in each frame. In addition, we use the Skeleton Detection module to detect the heads of the subjects and their pairs of hands.

While [34] used an initial keypoint detector to produce noisy labels in multiple views of the hand to detect parts of the hand, we use an initial keypoint detector to detect the joints in the body. Unlike [34], which used multiple cameras and a controlled environment, the proposed method in this thesis uses one camera, and it does not require a controlled environment.

The advantage of the approach in [33] and the proposed approach in this thesis in comparison to [29] and [35] is that the user does not require to stand while performing the gesture, thus the proposed approach is suitable for real-time implementation.

We have combined two approaches to human-robot interaction: a smart glove [31] and an image processing classification method [36]. The proposed system in this thesis recognizes the gestures from the smart glove using an SVM. The SVM uses data from flex sensors and an MPU and simultaneously classifies right-hand gestures in each frame with three validation modules to validate and improve the performance of the Hand Gesture Classification module. The SSD model is used for hand detection due to its simple training process, suitable speed, and accuracy, while the CNN model is used for gesture recognition. For the multi-subject environment, we add one more module to the algorithm that 
detects all present subjects in each frame and labels their bodies to identify their heads and hands. Afterward, a facial recognition model identifies the main subject and then an R-CNN determines the main subject's pair of hands. Finally, the CNN classification module classifies the user's right- or left-hand gestures (in the proposed implementation, we classify the right-hand gestures).

The R-CNN for object detection consists of several steps [37]. The category-independent region proposals are extracted from the captured input images. The fixed-length features are then calculated and obtained from region proposals using a large CNN model. Next, an R-CNN classifies each feature by feeding the data from the last layer into an SVM.

In [11], a Haar feature-based AdaBoost classifier has been used to categorize five different gestures for controlling a drone. While the accuracy of their framework is highest when the drone's operator posed within a distance of $3 \mathrm{ft}$, which is the sensing range of the camera used in the study, the proposed method works at its highest accuracy when the operator performs a gesture within the camera's sensing range, which is $8.5 \mathrm{ft}$.

Wearable gadgets that measure body movements are part of a rapidly growing field. The data collected from hand movements and gestures can be converted into voices or commands. For instance, those with speech impairments have difficulty communicating in a society in which most people do not understand sign language, similar to the glove in [38], the proposed glove converts sign language gestures into predefined commands. Their glove maps sign language to speech output by recognizing the gesture made by the hand. The proposed smart glove in this thesis uses exact parameter values to determine a command instead of a threshold to determine a gesture.

There are several gadgets that provide human-machine interaction, such as joysticks, buttons, keypads, keyboards, and gloves. The most significant advantages of smart gloves are their compatibility with natural human movement and their smooth fluctuation with three degrees of freedom. Therefore, using finger curvature and hand angles from smart gloves is an elegant method for human-machine interaction in controlling robot movements. Authors in [39] made a connection between wearable gadgets and non-wearable 
methods, such as joysticks, trackballs, mouse position, and mouse velocity, in random applications. The results show that compared to non-wearable gadgets, the robotic glove is more consistent with natural hand movements, has better interaction, and is closer to what the user expects from the behavior of a robot.

Furthermore, Table 2.2 provides a comparison between the proposed method and similar methods based on specific criteria such as the number of gestures, classifier accuracy, validation modules, ability to increase the number of gestures, adaptability with a new user, type of classifiers, and the source of interaction between the user and the system. As shown in Table 2.2, while no other method includes validations and the user must be alone in front of the camera, in our method there are three layers of validation and the method allows for multi-subjects where the algorithm can detect the main subject.

Table 2.2: Comparison between the proposed method and similar approaches.

\begin{tabular}{|c|c|c|c|c|c|}
\hline Other Approaches & \multirow{2}{*}{ Method [3] } & \multirow{2}{*}{ Method [4] } & \multirow{2}{*}{ Method [5] } & \multirow{2}{*}{ Method [6] } & \multirow{2}{*}{ Proposed Method } \\
\hline Criteria & & & & & \\
\hline Number of Gestures & $\begin{array}{c}13 \\
\text { Continuous } \\
\text { Commands }\end{array}$ & $\begin{array}{c}7 \\
\text { Continuous } \\
\text { Commands }\end{array}$ & $\begin{array}{c}9 \\
\text { Continuous } \\
\text { Commands }\end{array}$ & $\begin{array}{c}13 \\
\text { Continuous } \\
\text { Commands }\end{array}$ & $\begin{array}{l}20 \\
\text { Continuous and Discrete } \\
\text { Commands }\end{array}$ \\
\hline Increasing the Gestures & $\checkmark$ & $x$ & $\checkmark$ & $x$ & $\checkmark$ \\
\hline Classifier & $\mathrm{P}-\mathrm{CNN}$ & $\begin{array}{l}\text { No Classifier } \\
\text { Just Filtering }\end{array}$ & $\mathrm{CNN}$ & MLP & $\begin{array}{c}\text { Right Hand: SSD }+ \text { CNN }+ \text { R-CNN } \\
\text { Left Hand: SVM }\end{array}$ \\
\hline Classifier Accuracy & $91.9 \%$ & $82 \%$ & $81.5 \%$ & $92 \%$ & $94.28 \%$ \\
\hline $\begin{array}{l}\text { Adaptability with } \\
\text { New Users }\end{array}$ & $\checkmark$ & $x$ & $x$ & $x$ & $\checkmark$ \\
\hline Real-Time Processing & $\checkmark$ & $\checkmark$ & $\checkmark$ & $\checkmark$ & $\checkmark$ \\
\hline Output Validation & $x$ & $x$ & $x$ & $x$ & $\checkmark$ \\
\hline Commands Sources & Camera & Camera & $\begin{array}{c}\text { Wearable } \\
\text { IMU and MMG Sensors }\end{array}$ & $\begin{array}{l}\text { Wearable Accelerometer } \\
\text { and Gyroscope Sensors }\end{array}$ & $\begin{array}{c}\text { Camera and Wearable } \\
\text { MPU and Flex Sensors on a Glove }\end{array}$ \\
\hline $\begin{array}{l}\text { Independent of the } \\
\text { Surrounding Environment }\end{array}$ & $\checkmark$ & $x$ & $\checkmark$ & $\checkmark$ & $\checkmark$ \\
\hline
\end{tabular}




\section{Chapter 3}

\section{System Components}

\subsection{Introduction}

The programming languages $\mathrm{C}++$ and Python are used to program the proposed method, and CoppeliaSim software is used as the simulation environment based on real-world characteristics. Furthermore, for hardware, we use an Arduino UNO as the smart-glove processing unit. We also use flex sensors and an MPU 6050 as sensors that measure hand fluctuations. We use a Blue tooth module as the signal transmitter between the smart glove and the main processor in the robot, which is an NVIDIA graphics processing unit (GPU). This chapter presents an overview of the system components that we use in this project.

In the Chapter 4, the structure of the smart glove, the hand recognition module, and the validation modules are explained.

\subsection{System Components}

\subsubsection{Arduino}

To recognize left-hand gestures with a wearable gadget, smart glove, we require a programmable MEMS such as an Arduino UNO (Figure 3.1) which contains analog pins that read the flex sensor signals. Additionally, the Arduino UNO microprocessor is Bluetooth 
compatible, that is used for wireless transmission of data and commands from the smart glove to the main processor, an NVIDIA GPU, in the robot.

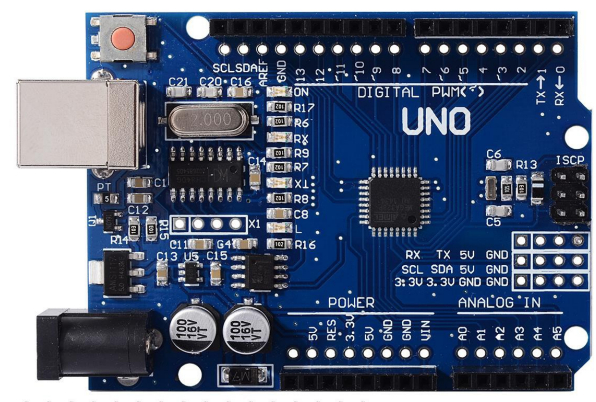

Figure 3.1: Arduino UNO board.

Arduino UNO is an open-source electronic board which is empowered by easy-to-use hardware and software. The programming language for Arduino UNO is $\mathrm{C}++$, and we program the Arduino to calibrate the flex sensors, read the flex sensors and MPU signals, and transmit the data to the main processor. This model of Arduino has an ATmega 328P microprocessor, which has 14 digital input/output pins, six analog inputs, a $16 \mathrm{MHz}$ quartz crystal, and a reset button.

\subsubsection{Flex Sensors}

The tool that we use to measure the finger bending value of the left hand consists of four flex sensors. Flex sensors produce various voltages when they bend, and we can measure the curvature in every finger by collecting the signals produced from this bending. Figure 3.2 shows that the flex sensors which are used in this thesis have two pins. In the proposed circuit, as shown in Figures 3.3 and 3.4, the positive pins are connected to each other and then connected to one of Arduino analog pins, and the negative pins are connected to the Arduino analog pins separately. In the proposed implementation, four flex sensors are implemented on the glove to measure the bending value of four fingers: the thumb, the index, the middle, and the ring fingers.

To shift the output voltage of the flex sensors to a range that the Arduino can manage with minimum fault and without damaging the board, we use a voltage divider, which is 


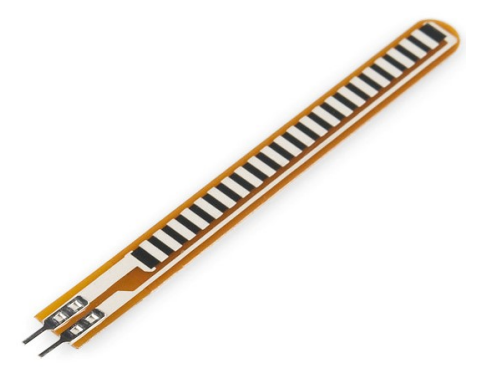

Figure 3.2: Flex sensor with two pins for producing voltage on them.

illustrated in Figure 3.3a.

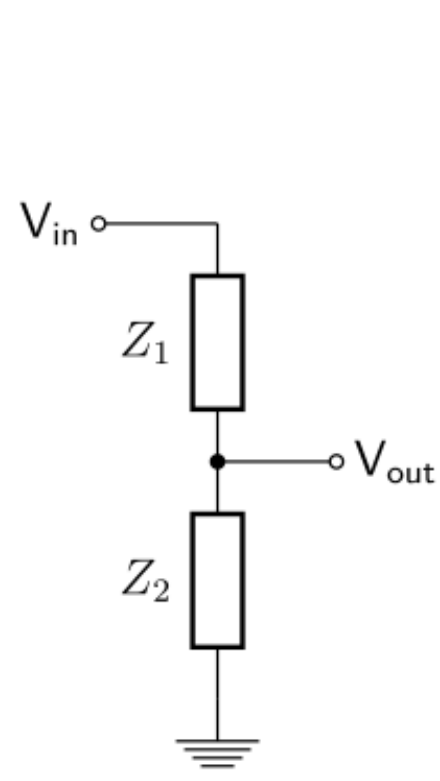

(a) Voltage divider circuit.

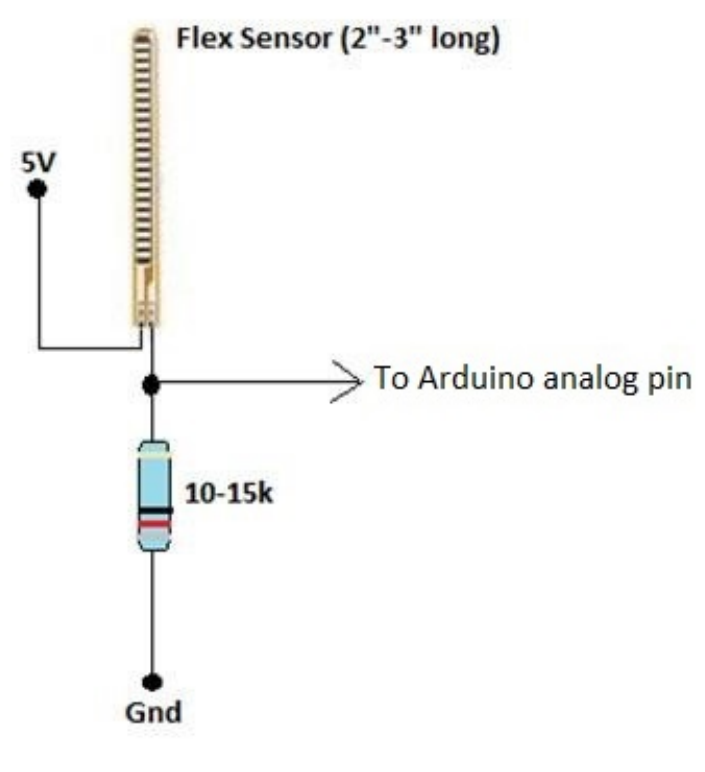

(b) Circuit of a flex sensor connection.

Figure 3.3: Flex sensors calibration diagram.

The voltage divider and the flex sensor are connected as shown in Figure 3.3b. In the proposed smart glove, all the flex sensors' positive pins are connected to one of the Arduino 5 volt pins. Furthermore, the rest of the analog pins of the flex sensors are coupled with $10 K \Omega$ resistors. For all the flex sensors, the other resistor pins are connected to the Arduino ground (GND) pin. Figure 3.4 shows that the Arduino analog pins are connected to the common connection between the flex sensors and resistors, and the other flex sensor pins are connected to the Arduino 5 volt pin.

The flex sensors are calibrated through the Arduino program. Since these sensors are 


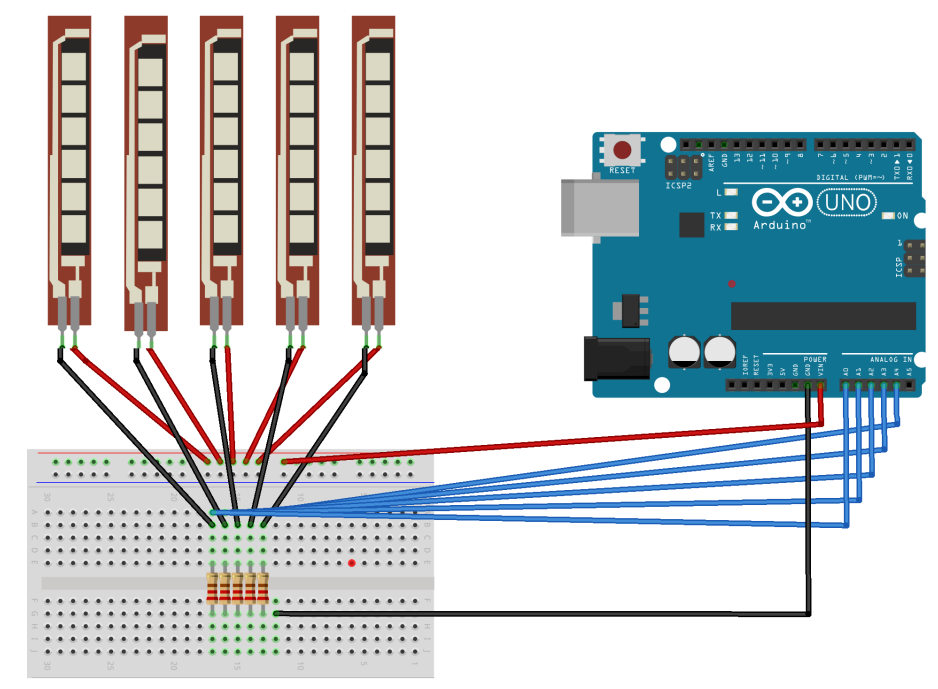

Figure 3.4: Connection between all flex sensors and Arduino pins.

used to measure the bend in every finger of the left hand to recognize its gesture, the maximum value is set when the fingers are bent, and the minimum value is set when the fingers are completely straight.

\subsubsection{Arduino Integrated Development Environment}

The Arduino integrated development environment (IDE) makes it easy to write $\mathrm{C}++$ code and upload it to the board. The code is written in two parts. The first part is run once at the beginning of the process and calibrates the flex sensors and the MPU. The second part is run after the first part and continues running for the rest of the process.

\section{I) First Part, Calibration}

- Flex sensors calibration: At the beginning of the process, this part of the program calibrates the flex sensors in two separate steps. Figures $3.5 \mathrm{c}$ and $3.5 \mathrm{~d}$ show that the first step is opening the fingers to set the minimum values to zero, and the second step is forming a fist with the fingers to set the maximum value of each sensor signal to $100 \%$.

○ MPU calibration: To define the the direction of the user's hand and to establish 
the zero angles of the hand, the user must hold the hand steady and perpendicular to the body and parallel to the ground.

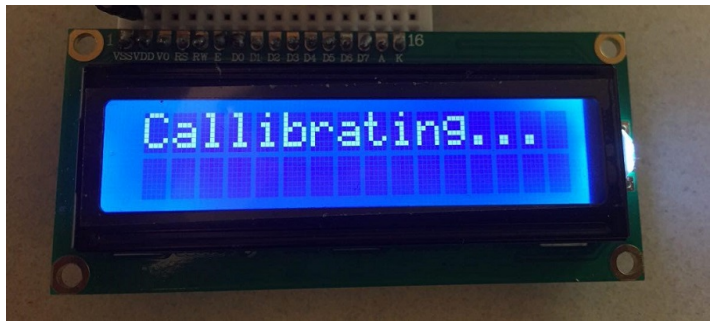

(a) Calibrating start.

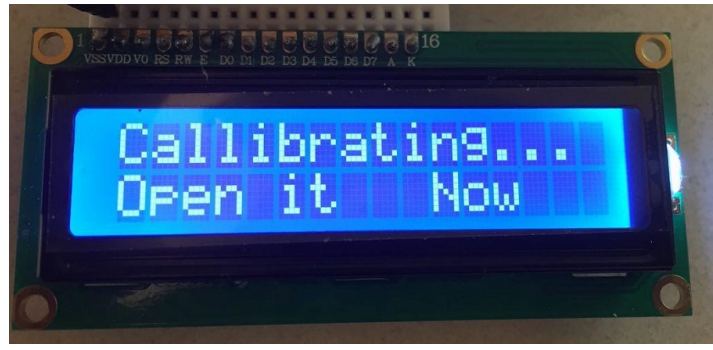

(c) Flex sensor calibration: setting the zeros.

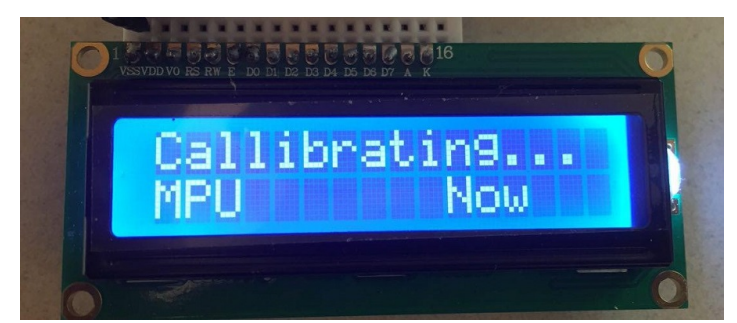

(b) Motion-processing unit calibration.

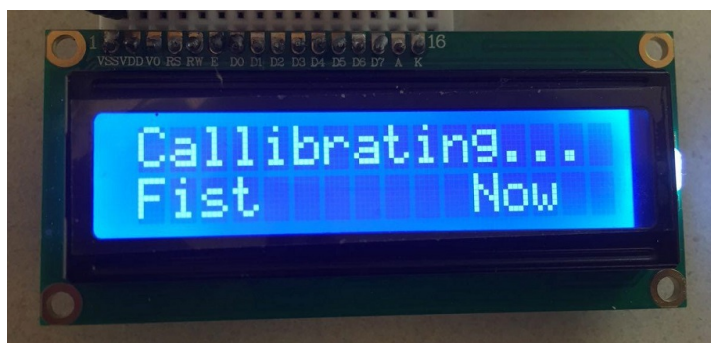

(d) Flex sensor calibration: fixing the maximums.

Figure 3.5: Calibration process: MPU module and the flex sensors.

\section{II) Second Part, Reading and Transmitting the Generated Signals}

○ Reading the flex sensor signals: After the calibration step, with any bend in the fingers, the flex sensors begin generating signals. Subsequently, the Arduino reads the produced signals through its analog inputs and converts them into the calculated minimum and maximum ranges as shown in Figure 3.6.

○ Reading the MPU values: With any fluctuation in the left hand at various angles, the MPU produces various signals, and the Arduino reads them through its analog pins, passes them through a filter, and then transmits them.

- Transmitting data: After reading the signals produced through the analog pins, the Arduino transmits the data through a Bluetooth module to the main processor. 


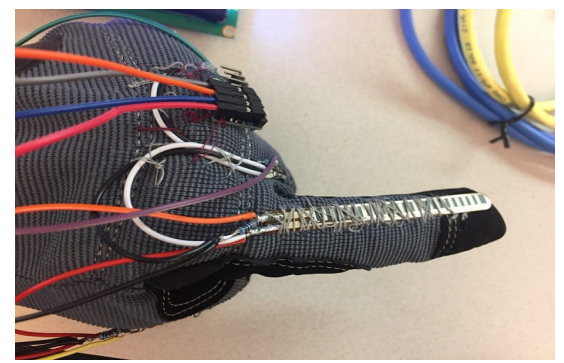

(a) Pointing gesture.

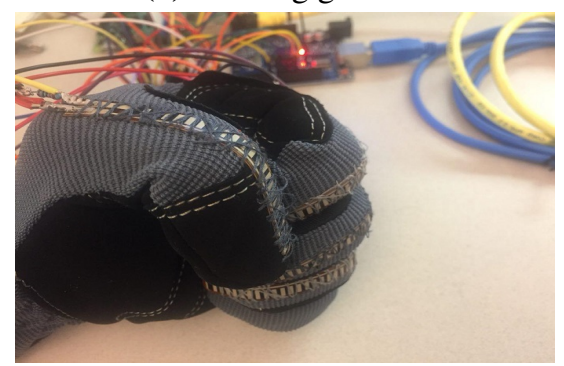

(c) Fist gesture.

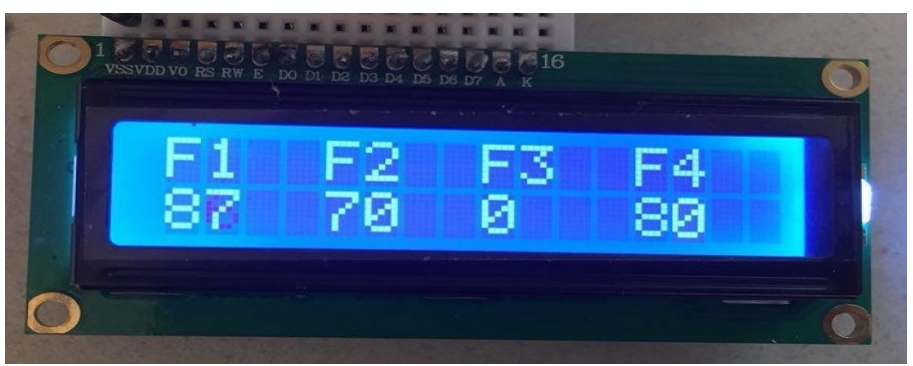

(b) Sensors output values for pointing gesture.

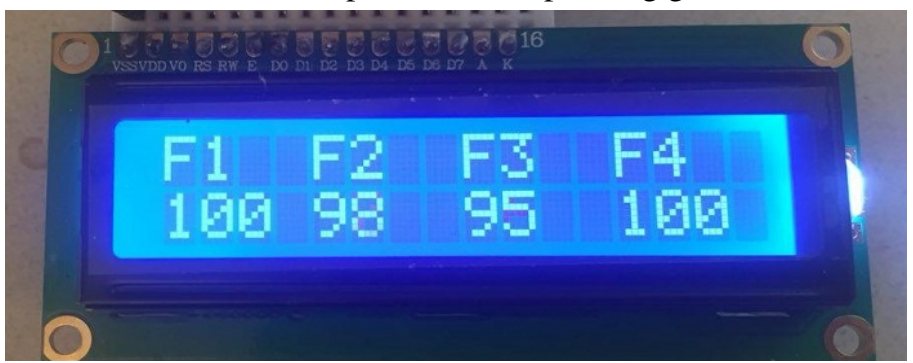

(d) Sensors output values for fist gesture.

Figure 3.6: Gestures with the smart glove. Bending values for four flex sensors on the glove are shown as F1, F2, F3, and F4 for four fingers of the left hand.

\subsubsection{MPU 6050}

The MPU 6050 sensor (Figure 3.7) contains a micro-electromechanical system accelerometer and a MEMS gyro in a single board. It is highly accurate, as it contains 16-bit analog-to-digital conversion hardware for each channel. Therefore, it captures the $x$-, $y$-, and $z$ - axis simultaneously. The sensor also uses a I2C-bus to interface with the Arduino UNO.

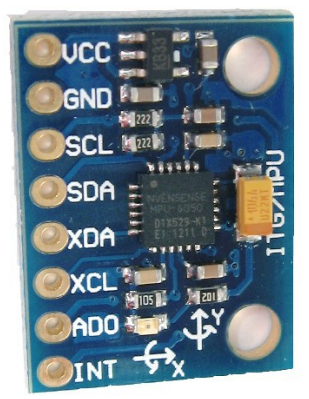

Figure 3.7: MPU 6050.

This sensor helps to measure the angle of the left hand in the $x-, y$-, and $z$-axis. By using 
the MPU pins which are SDA, SCL, VCC, and GND pins, we can read the MPU signals. The signals thought these pins are analog, so we connect them to the Arduino analog pins as well as the flex sensors.

\subsubsection{Bluetooth Module}

We use a bluetooth module as the data transmitter to transmit the collected data from the flex sensors and the MPU to the main processor, which is located in the robot, Figure 3.8 .

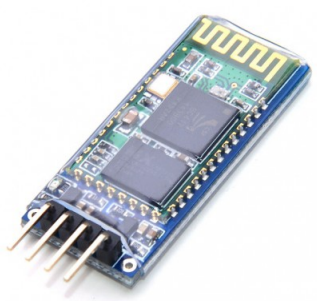

Figure 3.8: Arduino UNO Bluetooth module.

Additionally, the smart glove can be extended for use with both hands. We can implement all the equipment and circuits individually for each hand and then transfer data from one hand to another through a Bluetooth module and from the second hand, we can transmit the collected data from both hands to the main processor.

The deployed smart glove's equipment are demonstrated in Figure 3.9.

\subsubsection{Webcam}

This thesis proposes an adaptable human-robot interface that uses two types of human-computer interactions, one of which is an image processing technique for right-hand gesture recognition. The image processing technique requires a webcam as its input device to capture the frames.

The cameras that we use for this project are the AUSDOM AW335 Full HD1080p webcam (Figure 3.10a) for single-subject scenarios and a LOGI HD 1080p webcam (Figure 


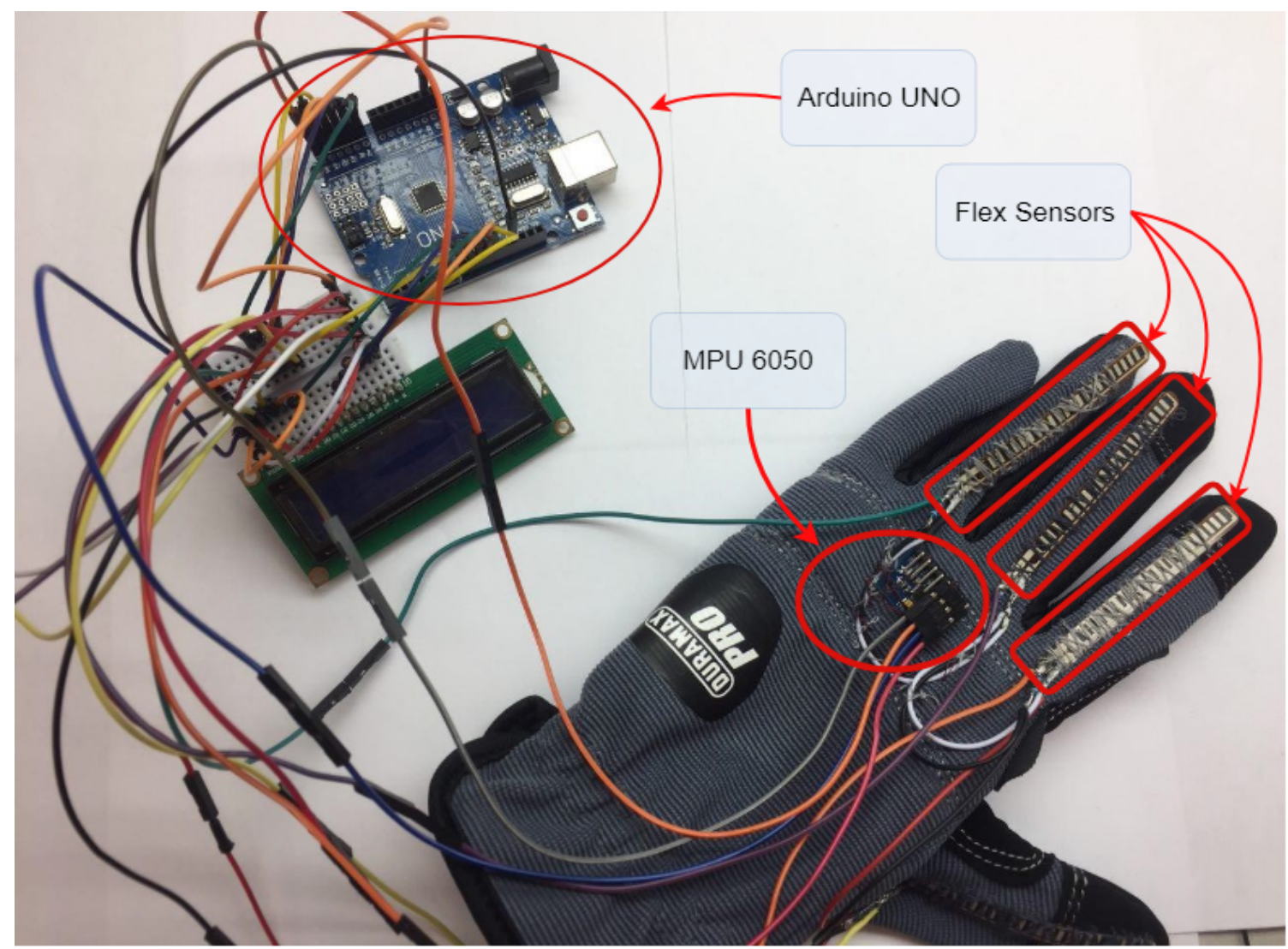

Figure 3.9: Smart glove.

3.10b) for multi-subject environments. The input frames are taken through these webcams and sent to the neural networks to capture a hand and its gestures.

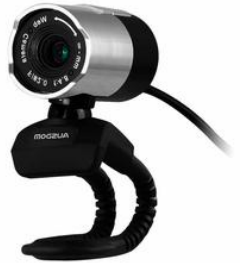

(a) AUSDOM AW335 Full HD1080p webcam.

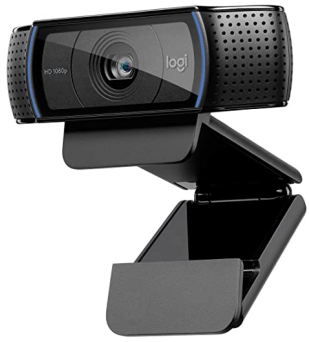

(b) LOGI HD 1080p webcam.

Figure 3.10: Cameras used in the project. 


\subsubsection{NVIDIA GPU}

The proposed image-based human-robot interaction model includes an SSD network to recognize a user's hand. It also includes a $\mathrm{CNN}$ to classify the gestures of the detected hand in a single-subject environment and an R-CNN to detect the subjects in the input frame in a multi-user environment. Further, it has a facial recognition module to recognize the main subject and a CNN classifier to classify the main subject's right-hand gestures. Thus, a powerful GPU is required to run machine-learning (ML) and deep-learning (DL) models such as CNN and R-CNN concurrently. For this purpose, we chose the NVIDIA Jetson AGX Xavier GPU (Figure 3.11).
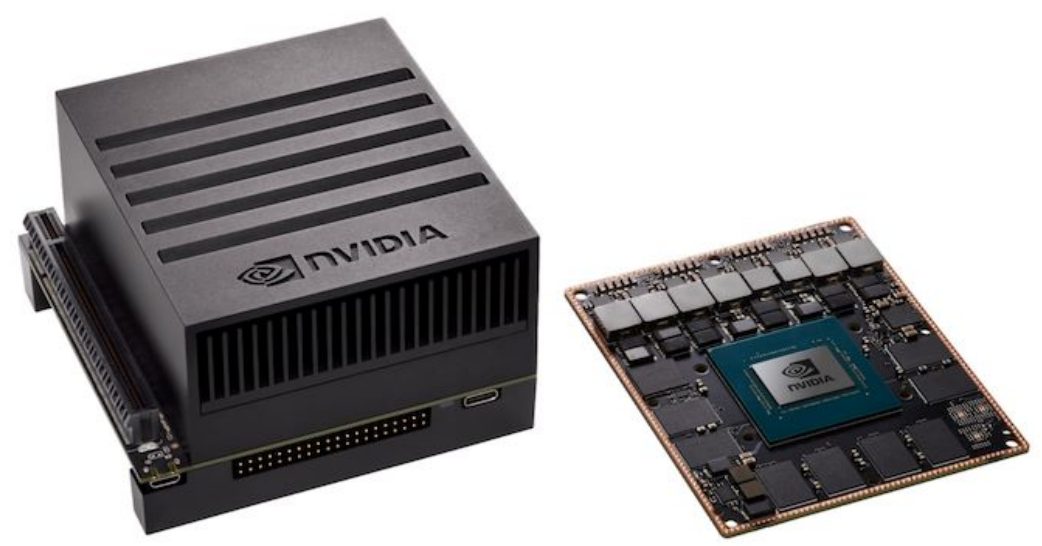

Figure 3.11: The main processor on the robot, the NVIDIA GPU.

This Linux-based GPU with 512 cores and 32GB memory is capable to implement ML and DL models and build an adaptable human-robot interface that uses two types of human-computer interactions concurrently. Utilization of this GPU enables the system to reach a reasonable speed in the proposed algorithm run-time without any timeout and decreasing in accuracy. 


\section{Chapter 4}

\section{System Implementation}

\subsection{Introduction}

This chapter addresses the structure of the smart glove, the hand recognition module, and the validation modules. We show block diagrams for the system and the recognition sections, pseudo-code for validation process, and the configuration of the CNN.

\subsection{Smart Glove}

The proposed smart glove converts hand gestures into a set of predefined commands. The microprocessor gathers all the raw data to analyze them in separate units. Figure 4.1 shows the component's locations on the smart glove. The flex sensors are placed on ring, middle, index, and thumb fingers, and the MPU module is placed on the back of the glove. The Arduino is place on the wrist, and the bluetooth Module and the battery are placed on front side of the smart glove.

\subsubsection{Flex Sensors}

Flex sensors generate voltage signals as a function of applied strain. First, the flex sensors and the MPU must be calibrated. To calibrate the flex sensors, the fingers must be 

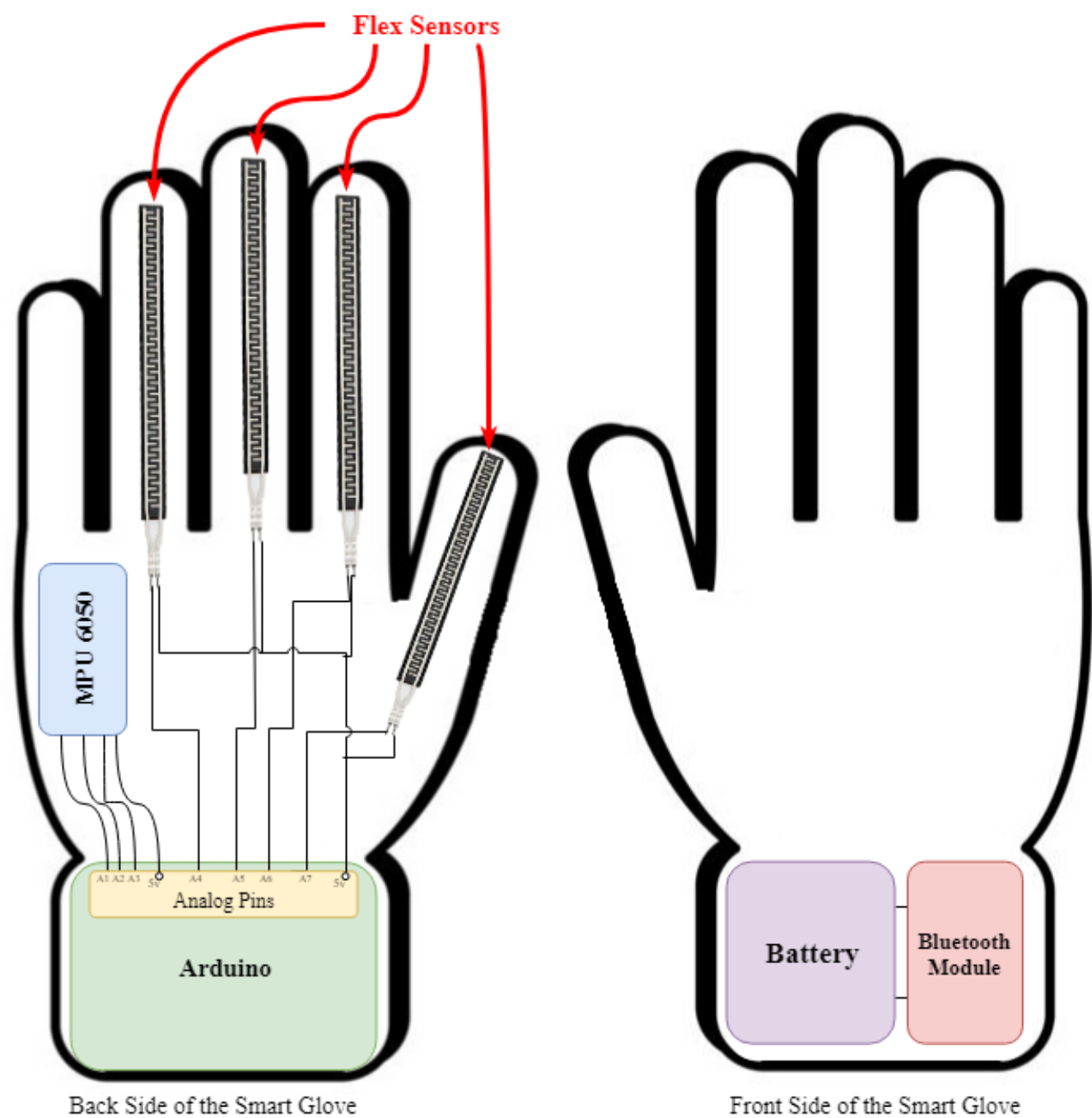

Figure 4.1: Smart Glove designed in this project.

kept fully open, and the algorithm then assigns an output value of zero to each of the flex sensors. The fingers must then be closed, and the algorithm assigns a value of $100 \%$. To calibrate the MPU, the user must keep the smart glove in a stable position and parallel to the ground. After reading the data, the microprocessor broadcasts the data via a transmitter to the Flight Controller and the System Validation module.

The smart glove operates in two different modes simultaneously. The first mode considers each finger as an on/off switch, and the second mode uses the exact bending values of each flex sensor. 


\section{Flex Sensors as On/Off Switches:}

The smart glove supports 16 different gestures. To analyze these gestures, an SVM model with a linear kernel is used. The output of the SVM model determines whether each flex sensor is on or off and the command that four of them represent. The algorithm then uses the exact bending value of each flex sensor in the second mode of the smart glove. These commands are separated into two class types to communicate with the System Validation module and the Flight Controller, which are going to be explained in the next section.

\section{Bending Value:}

This mode determines the first coefficient for the chosen command. For instance, if Gesture \#2 represents turn left, the finger-bending value determines the angular velocity. The system converts each gesture into binary code. Each digit represents a flex sensor, and the SVM output determines whether each digit is on or off. For example, Gesture $\# 2$, or the pointing gesture as shown in Figure 3.6(a), is coded in binary as 0010. After determining each digit, the system considers the changes of $r s_{j}(t)$ for the corresponding flex sensor $j$ from the moment that flex sensor is activated $t_{0}$ (those sensors with value 1) in each sampling time $t$ as

$$
r s_{j}\left(t_{n}\right)=f_{j}\left(t_{n}\right)-f_{j}\left(t_{0}\right)
$$

where $r s_{j}\left(t_{n}\right)$ is the change between time step $t_{n}$ and $t_{0}$. The bending values for flex sensor $j$ between time steps $t_{n}$ and activation time $t_{0}$ are $f_{j}\left(t_{n}\right)$ and $f_{j}\left(t_{0}\right)$, respectively. We determine the reference velocity of the drone, $v_{G}\left(t_{n}\right)$, as

$$
v_{G}\left(t_{n}\right)=\frac{r s_{j}\left(t_{n}\right)}{\max \left\{f_{j}\right\}-\min \left\{f_{j}\right\}} v_{0}+v_{0}
$$

where $v_{0}$ is the drone's velocity before each classification and which we consider an initial velocity. Maximum and minimum bending values, $\max \left\{f_{j}\right\}$ and $\min \left\{f_{j}\right\}$, are calculated in the calibration step for each flex sensor. In the following sections, we show the effect of 
an activated flex sensor that corresponds to $f_{j}$ value.

For instance, in Figure 3.6, when the user makes a pointing gesture, the microprocessor gathers the bending value of each flex sensor and then sends the data to the computation unit. An SVM model then classifies the collected data into one of the 16 possible left-hand gestures. Figure 4.2 shows the difference in value for each gesture. Descriptions of the generated gestures with the smart glove are provided in Table 4.1. Table 4.2 provides flex sensors features and specifications.

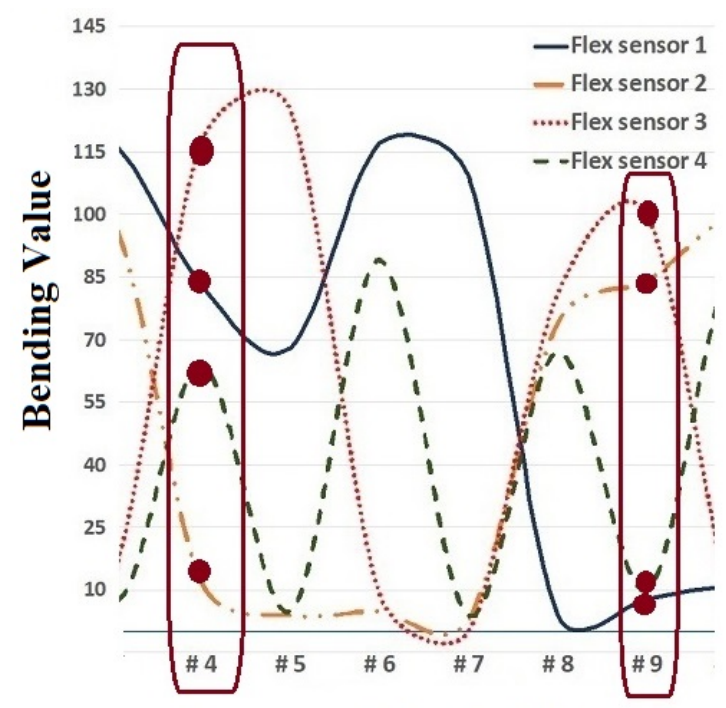

Gestures of the Left Hand

Figure 4.2: Finger's bending values of four flex sensors. As indicated on the horizontal axis, a combination of four bending values from the flex sensors represents a specific gesture. 
Table 4.1: Gestures for the smart glove and their corresponding functions.

\begin{tabular}{|c|c|}
\hline Gestures & Description \\
\hline Gesture \# 1 & land \\
Gesture \# 2 & turn left \\
Gesture \# 3 & turn right \\
Gesture \# 4 & increase the height \\
Gesture \# 5 & decrease the height \\
Gesture \# 6 & turn $180^{\circ}$ \\
Gesture \# 7 & move straight to forward \\
Gesture \# 8 & move straight to backward \\
Gesture \# 9 & reserved \\
Gesture \# 10 & reserved \\
Gesture \# 11 & reserved \\
Gesture \# 12 & reserved \\
Gesture \# 13 & reserved \\
Gesture \# 14 & reserved \\
Gesture \# 15 & penalty for the output \\
Gesture \# 16 & hover \\
\hline
\end{tabular}

Table 4.2: Flex sensors features and specifications.

\begin{tabular}{|l|c|}
\hline Features & Specifications \\
\hline Operating Voltage & $0-5 \mathrm{~V}$ \\
\hline Operating Temperature & $-45^{\circ} \mathrm{C}$ to $+80^{\circ} \mathrm{C}$ \\
\hline Flat Resistance & $25 K \Omega$ \\
\hline Resistance Tolerance & $\pm 30 \%$ \\
\hline Bend Resistance Range & $45 K \Omega$ to $125 K \Omega$ \\
\hline
\end{tabular}

\subsubsection{MPU 6050}

The MPU module, with its gyroscopic sensor, divides the space into three angle ranges: $0^{\circ}$ to $60^{\circ}, 60^{\circ}$ to $120^{\circ}$, and $120^{\circ}$ to $180^{\circ}$, as shown in Figure 4.3. The MPU generates different values by holding the hand in different angles along the $x$-axis, $y$-axis, and $z$-axis. These values are used as the second coefficients for the 16 hand gestures or as separate coefficients for a certain parameter. For instance, we can define the pitch axis ( $z$-axis) of the hand as a coefficient of the drone altitude. The Flight Controller uses the MPU correspond value to the $z$-axis as a coefficient of the drone altitude unless a command is 


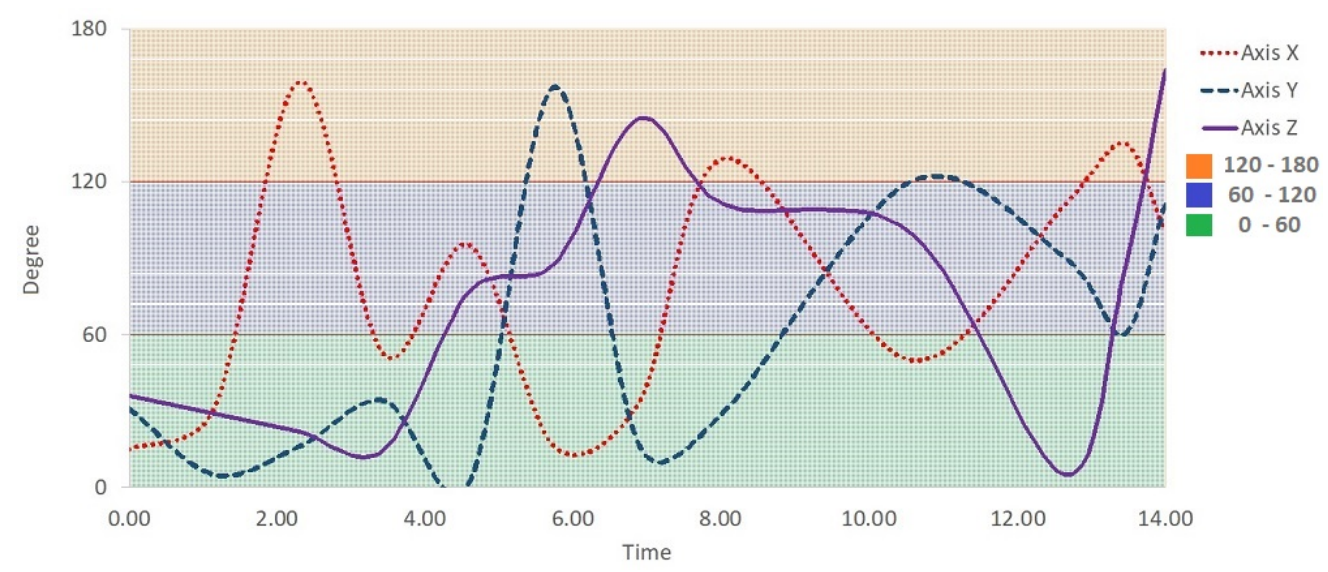

Figure 4.3: MPU response in three zones by fluctuating the smart glove.

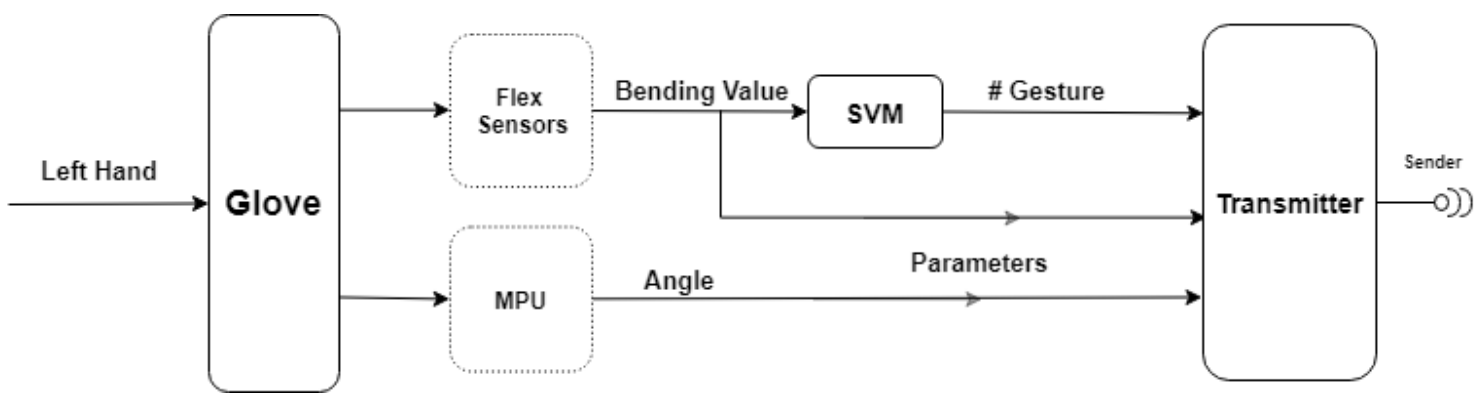

Figure 4.4: Gesture recognition module with the smart glove. This unit by using flex sensors calculates the bending value for each finger and then an SVM classifier classifies the gesture based on those bending value. An MPU module is used to calculate the angle of the left hand.

given to change the state of the drone. If the user holds the smart glove pitch angle in the third range $120^{\circ}$ to $180^{\circ}$, the altitude of the drone rises. If the user holds the smart glove pitch angle in the second range $60^{\circ}$ to $120^{\circ}$, the altitude does not change and if the pitch angle in the $z$-axis drops to the range $0^{\circ}$ to $60^{\circ}$, the altitude decreases.

Figure 4.4 shows the smart glove block diagram with all of its components. Figure 4.5 shows a simple GUI that gathers all the signals produced by the flex sensors and the MPU and shifts the gathered signals into a calibrated range. The GUI contains three sections: the SVM output, the flex sensor output, and the MPU output. 


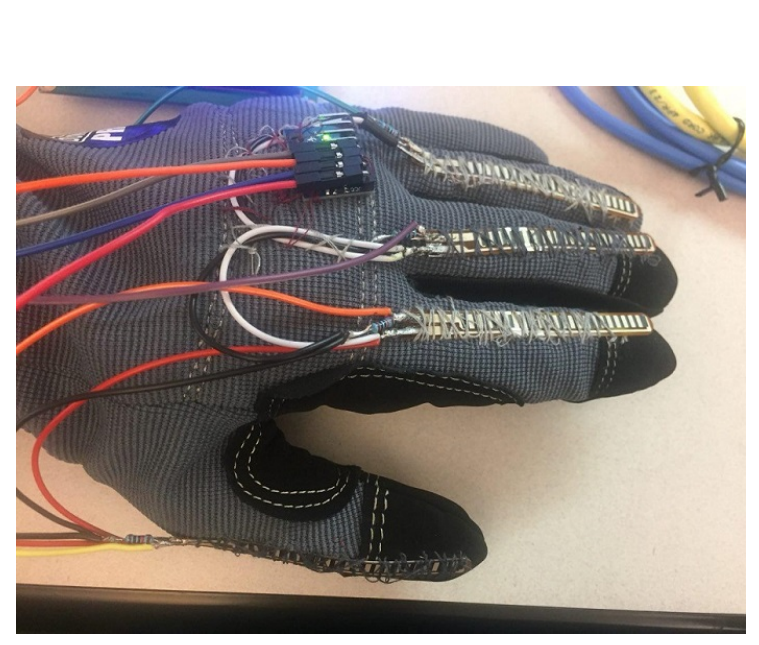

(a) Open palm gesture.

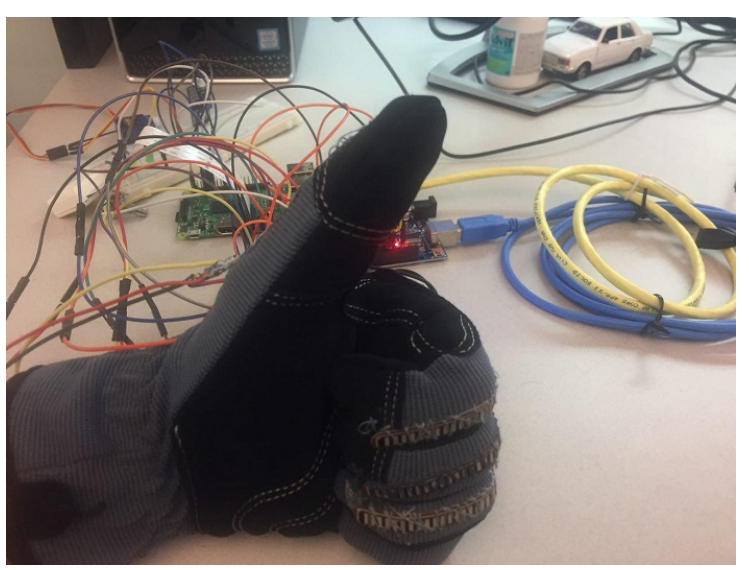

(c) Thumb up gesture.

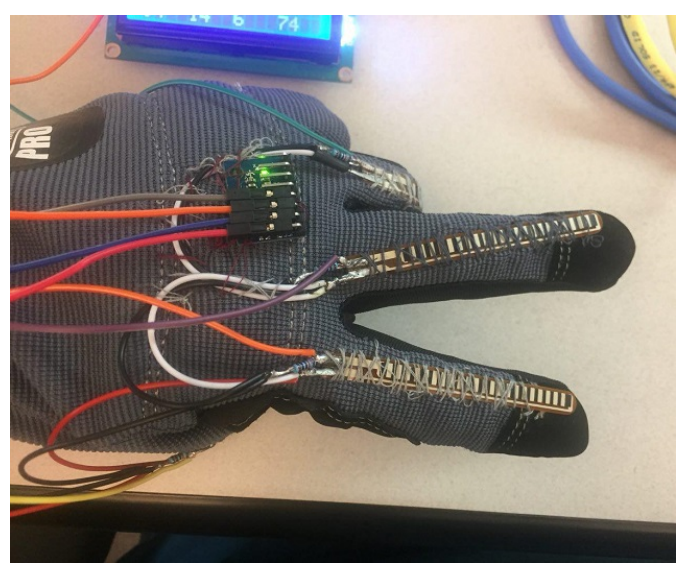

(e) Victory sign gesture.

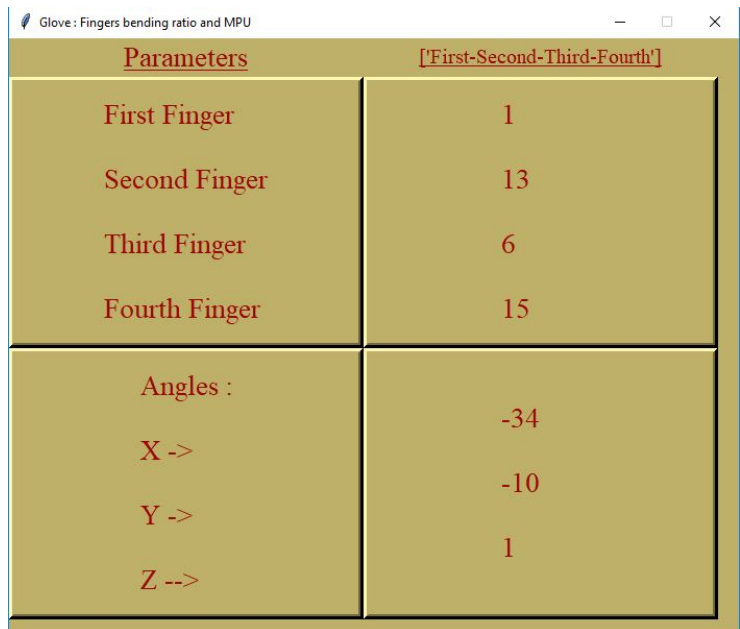

(b) Outputs for open palm gesture.

\begin{tabular}{|c|c|}
\hline Glove: Fingers bending ratio and MPU & ['---Fourth'] \\
\hline Parameters & 98 \\
\hline First Finger & 95 \\
Second Finger & 89 \\
Third Finger & 0 \\
\hline Fourth Finger & 92 \\
\hline Angles : & -97 \\
X -> & 0 \\
\hline Y -> & \\
\hline
\end{tabular}

(d) Outputs for thumb up gesture.

\begin{tabular}{|c|c|}
\hline \multicolumn{1}{|c|}{ Glove: Fingers bending ratio and MPU } & ['-Second-Third-'] \\
\hline Parameters & 78 \\
\hline First Finger & 0 \\
Second Finger & 0 \\
Third Finger & 48 \\
\hline Fourth Finger & 41 \\
\hline Angles : & 7 \\
X -> & -49 \\
\hline Y --> & \\
\hline
\end{tabular}

(f) Outputs for Victory sign gesture.

Figure 4.5: Smart glove gestures with the GUI. 


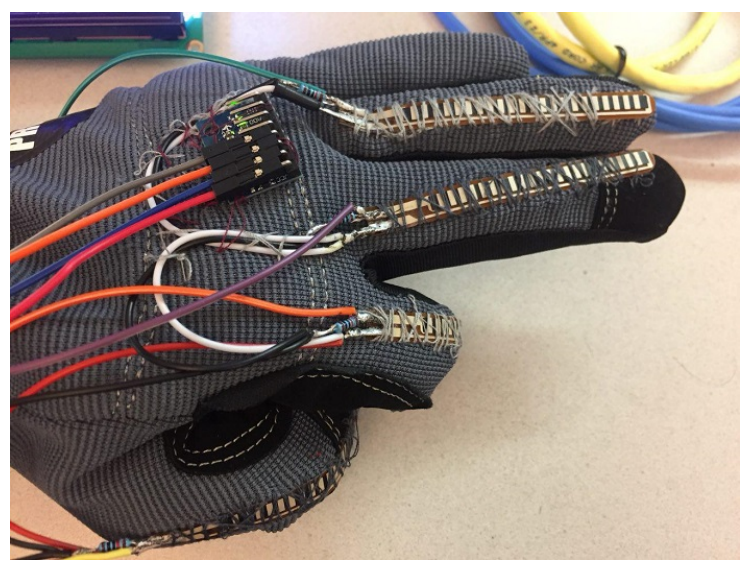

(a) First and second fingers are open.

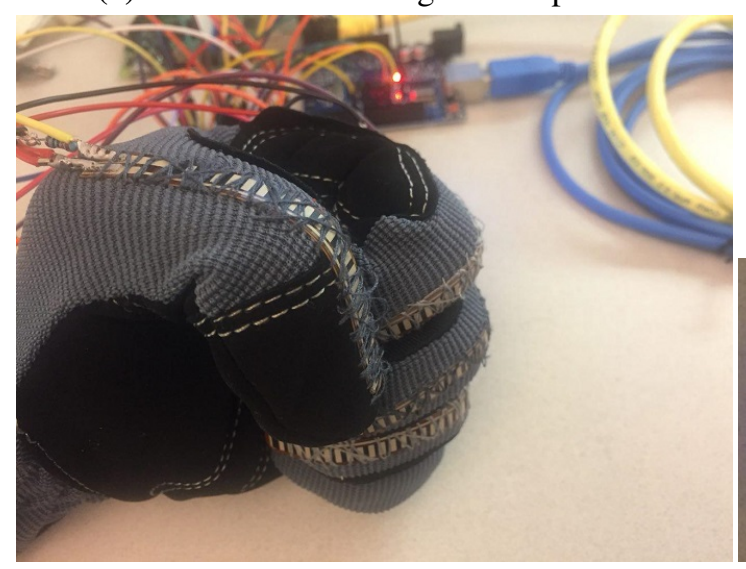

(c) Fist gesture.

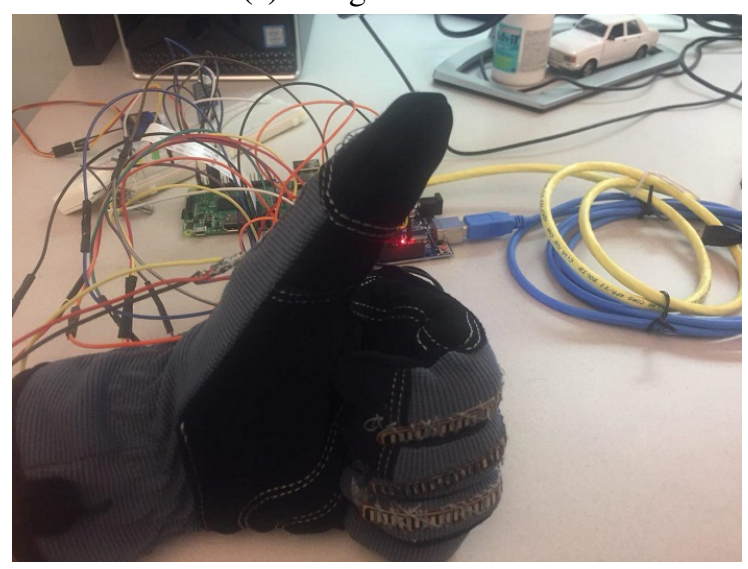

(e) Thumbs up gesture.

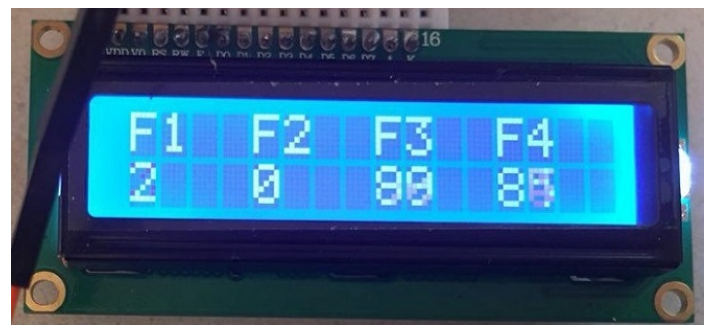

(b) Flex sensors outputs.

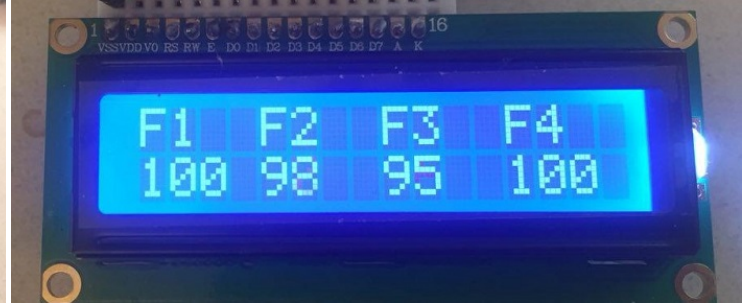

(d) All flex sensors outputs are maximum.

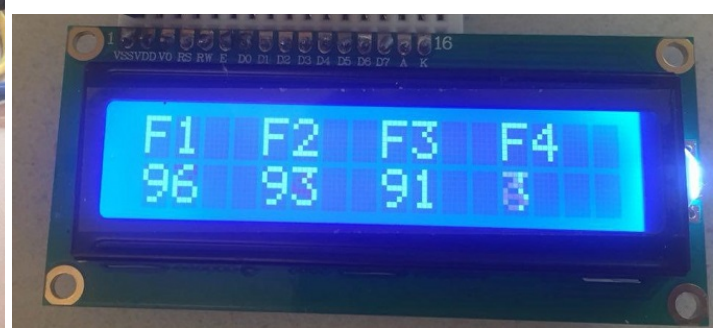

(f) Thumbs up flex sensors outputs.

Figure 4.6: Smart glove gestures and their flex sensors outputs. 


\subsection{Hand and Gesture Recognition in a Single-Subject Environment}

Block diagram in Figure 4.7 illustrates the gesture-based control system with four main units: (i) Human-in-the-Loop Validation, (ii) Hand Detection and Gesture Classification Unit, (iii) Flight Controller, (iv) and System Validation module. In the single-subject environment we use the hand detector to find the hand and the detected hand is then classified. Also, there is a System Validation module which activates a Retrainer module if the classifier has a low confidence in its results. Then, the Retrainer retrains the classifier until the classifier reaches a better confidence which can be reached even in one step.

Two types of gesture recognizers are used in this thesis to interact with an unmaned aerial vehicle: (i) a smart-glove method and (ii) an image processing method. Note that the classifier in the proposed method does not create time-series predictions; also, the proposed method in this thesis focuses mainly on static gestures (or postures) and not dynamic gestures.

The Hand Detection and Gesture Classification Unit in the single-subject case has three

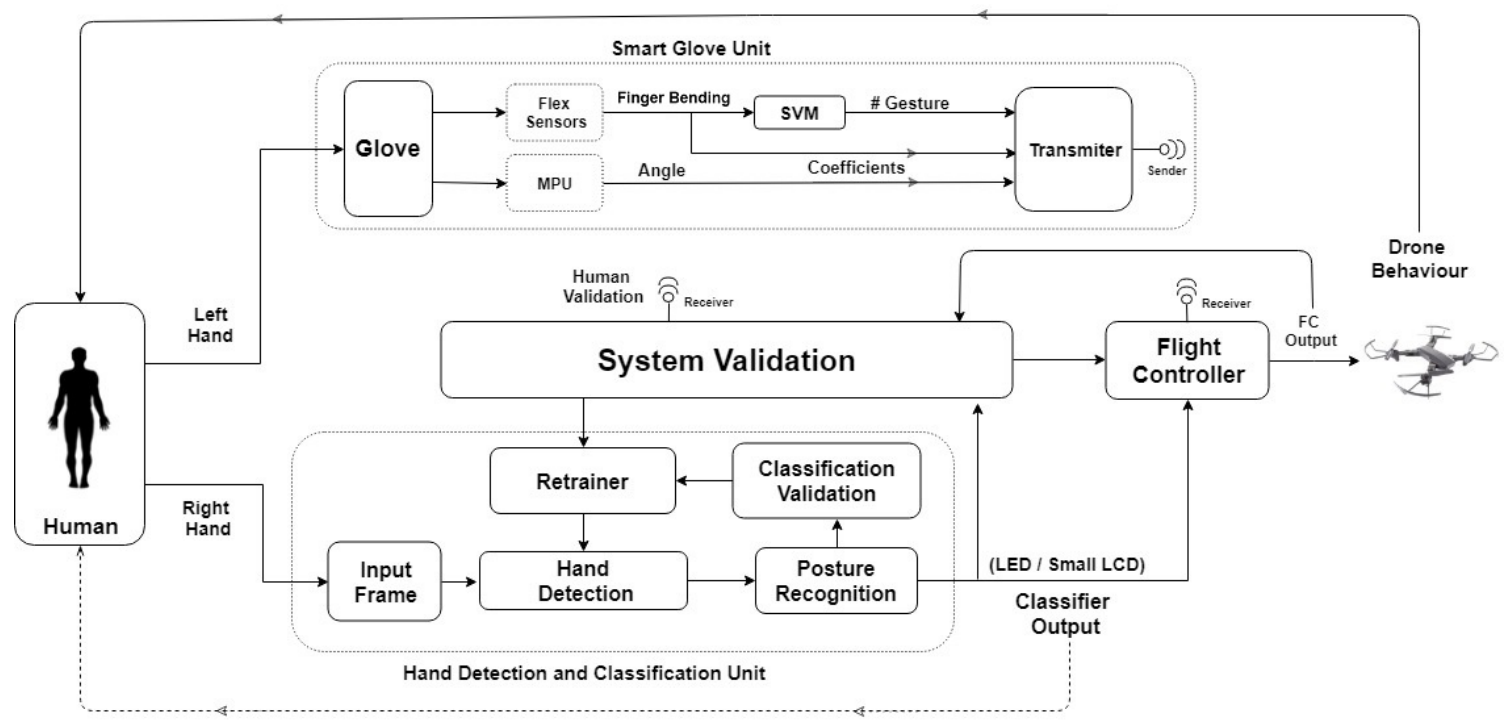

Figure 4.7: System block diagram for a single-subject environment, including three separate validation modules: (i) Classification Validation, (ii) System Validation, and (iii) human-in-the-loop validation. 
main modules: (i) the Hand Detection and Classifier module, (ii) the Classification Validation module, and (iii) the Retrainer module (Figure 4.8). The primary input to this unit is a gesture frame, and the main output is the classified gesture represented as a class. The classifier has an internal validation module, which checks and improves the classifier's performance.

We performed hand detection and gesture recognition in a single-subject scenario using the AUSDOM AW335 Full HD 1080p webcam. The inputs in the first stage were images from the camera. First, we converted input images from RGB to grayscale to reduced image dimensionality and thus accelerated the process. Later, these grayscale images were fed into the Hand Detection module to find and capture the hand, if there is one, in each image.

\subsubsection{Hand Detector}

The first step of gesture recognition is detecting a hand in the input frame, Figure 4.8. The algorithm uses the SSD model to detect the hand and the CNN model to classify the detected hand into one of the predefined classes. The classifier output maps to one of the predetermined commands such as take off, land, turn right, or turn left. The output command is then sent to the Flight Controller, which is used to execute the command. The command is then sent to the System Validation module and the Classification Validation module.

We chose the SSD model for hand detection because of its simple training and appropriate speed and accuracy [12]. We trained the SSD model with $N$ images of size of $n \times m$ pixels from various users who are in front of a white background showing one of the four hand gestures. After the hand is tracked, the SSD select a rectangular RoI of the detected hand. This RoI and the input frame from which it is extracted (input frame of the SSD) are flattened and sent into the next model for classification. Thus, to recognize various hand postures in each frame, the classifier does not check the entire frame; it checks only the position in the input frame that is related to the extracted RoI. This method is used to increase the accuracy and speed of the system. 


\subsubsection{Gesture Classifier}

We select the CNN model for the proposed gesture recognition step. Compared to its predecessors, the main advantage of $\mathrm{CNN}$ is that it automatically detects the essential features in each sample for each class without any human supervision. Furthermore, the proposed algorithm is designed to retrain and accept new classes, in which this feature is possible with CNN's retraining and pre-training features. Another advantage of using a $\mathrm{CNN}$ model as the classifier is the weight sharing feature of $\mathrm{CNN}$, which makes $\mathrm{CNN}$ more efficient in terms of memory and complexity. The CNN model consists of several layers of ANNs stacked in a cascading manner, [15]. To train the CNN model, we use an extensive database, as described in detail in Section 4.4.4. To train the classifier, we use the Keras library [40], which is a powerful library to build layers for each neural network model. By using the Keras library and CNN model, each detected RoI from the input frame is classified into one of the four available classes as shown in Figure 4.9.

The CNN model also uses a dropout technique and a sigmoid activation function to prevent overfitting [17]. The number of layers in the CNN model that we use in this thesis and the description of each layer are shown in Table 4.4, and the CNN model structure has

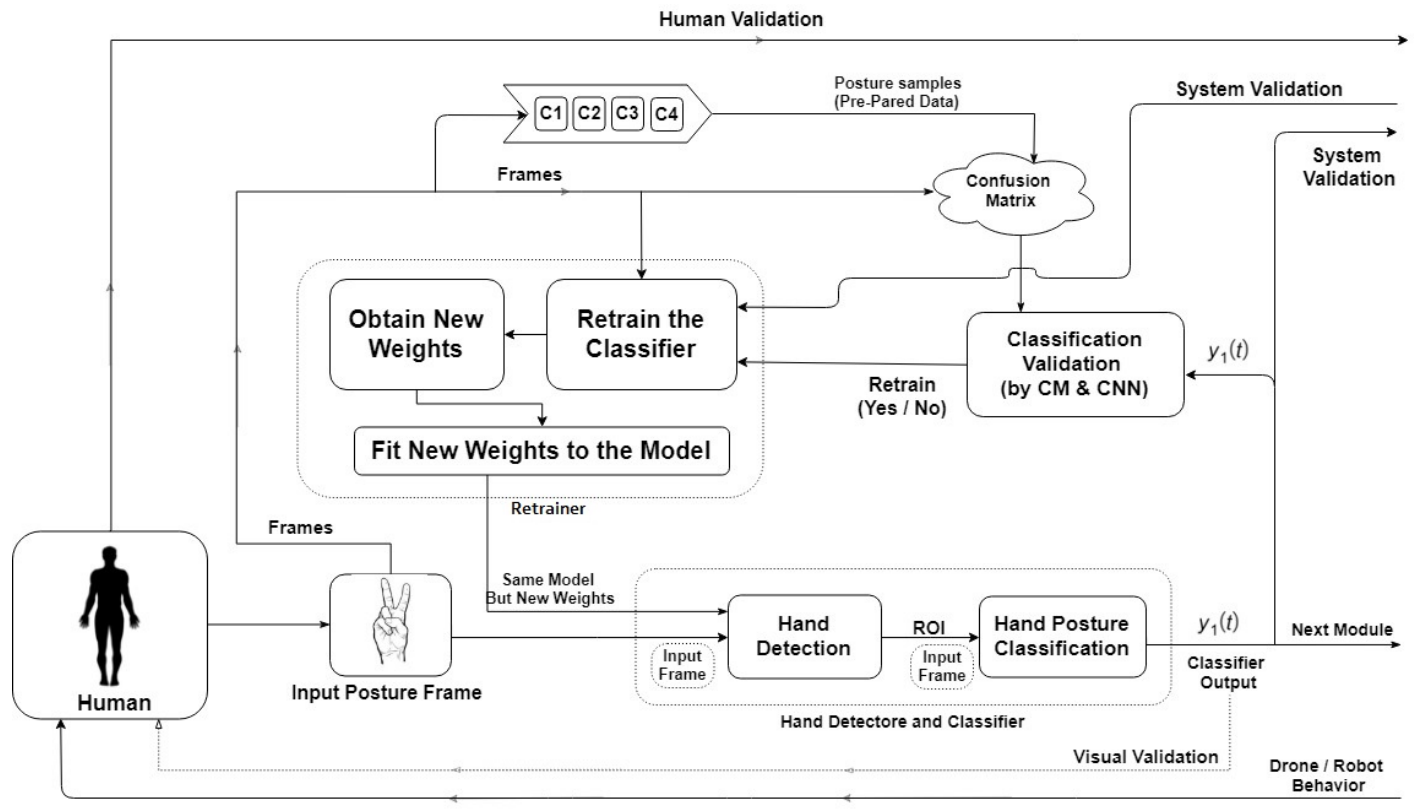

Figure 4.8: Hand Detection and Classification Unit block diagram in a single-subject environment. 


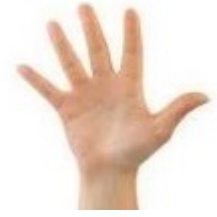

(a) Palm

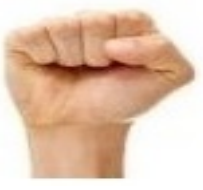

(b) Fist

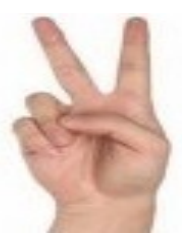

(c) Victory sign

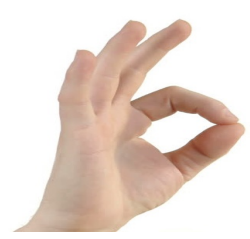

(d) OK sign

Figure 4.9: Four specified right-hand gestures as the webcam inputs, for classification. Each gesture represents a particular command that is given to the multi-rotor aerial vehicle as a control signal. The possible classes are (a) Palm (Class \#1): take off, (b) Fist (Class \#2): land, (c) Victory sign (Class \#3): turn left, and (d) OK sign (Class \#4): turn right.

been illustrated in Figure 2.2. Considering the dataset and the problem statement, during the experimentation phase, we chose the number of hidden layers in the CNN model. We increased the number of each type of layer until the classifier achieved our desired performance. The CNN's performance decreased as the number of layers increased; therefore, we reduce the number of that specific layer by one unit and use the result in the CNN architecture.

\subsubsection{Retrainer Module}

Figure 4.8 illustrates that the Retrainer module is activated in two cases: (i) when the Classification Validation module detects low confidence in the confusion matrix and (ii) when the System Validation module sends the retraining penalty signal. After the Retrainer module is enabled, as described in [13] for the single-subject environment, the Retrainer receives $S$ new images ( $S=5$ in our implementation) from the user as its input. The Retrainer module then retrains the running $\mathrm{CNN}$ model with the newly gathered data to obtain new weights. The module then fits the new weights to the CNN model and sends the $\mathrm{CNN}$ model to the classifier. Algorithm 1 illustrates the steps for the validation process in a single-subject environment. 


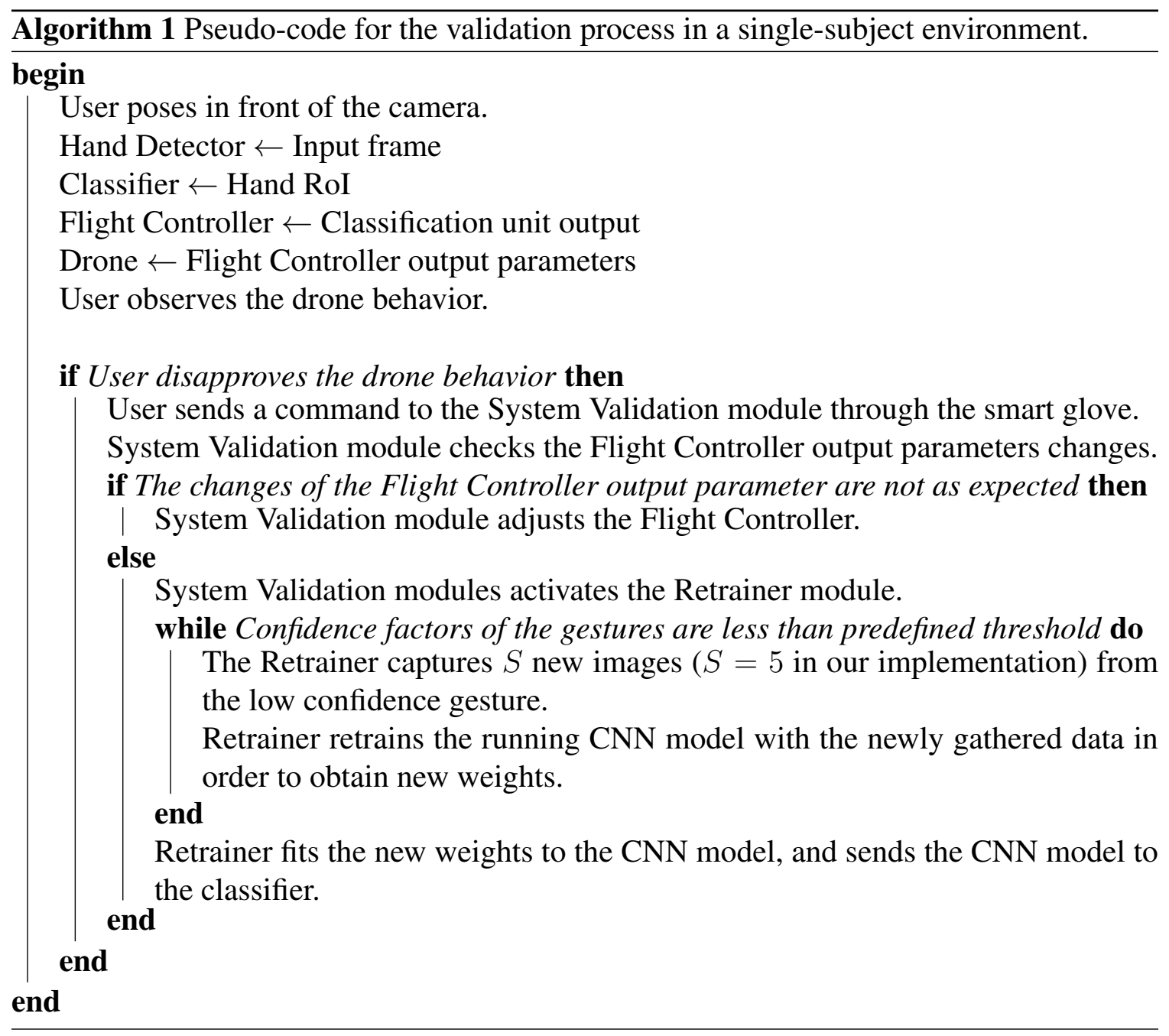

\subsection{Hand and Gesture Recognition in a Multi-Subject Environment}

We use the CNN model in both single- and multi-subject environments to classify a right-hand gesture into one of the classes illustrated in Figure 4.9. Block diagram in Figure 4.10 illustrates that the gesture-based closed-loop control system for multi-subject environments contains five main units and modules: (i) the Smart Glove unit, (ii) a Skeleton Detection module and a Face Recognition, (iii) a Gesture Classification unit with a Retrainer and a Classification Validation module, (iv) a Flight Controller, and (v) a System 


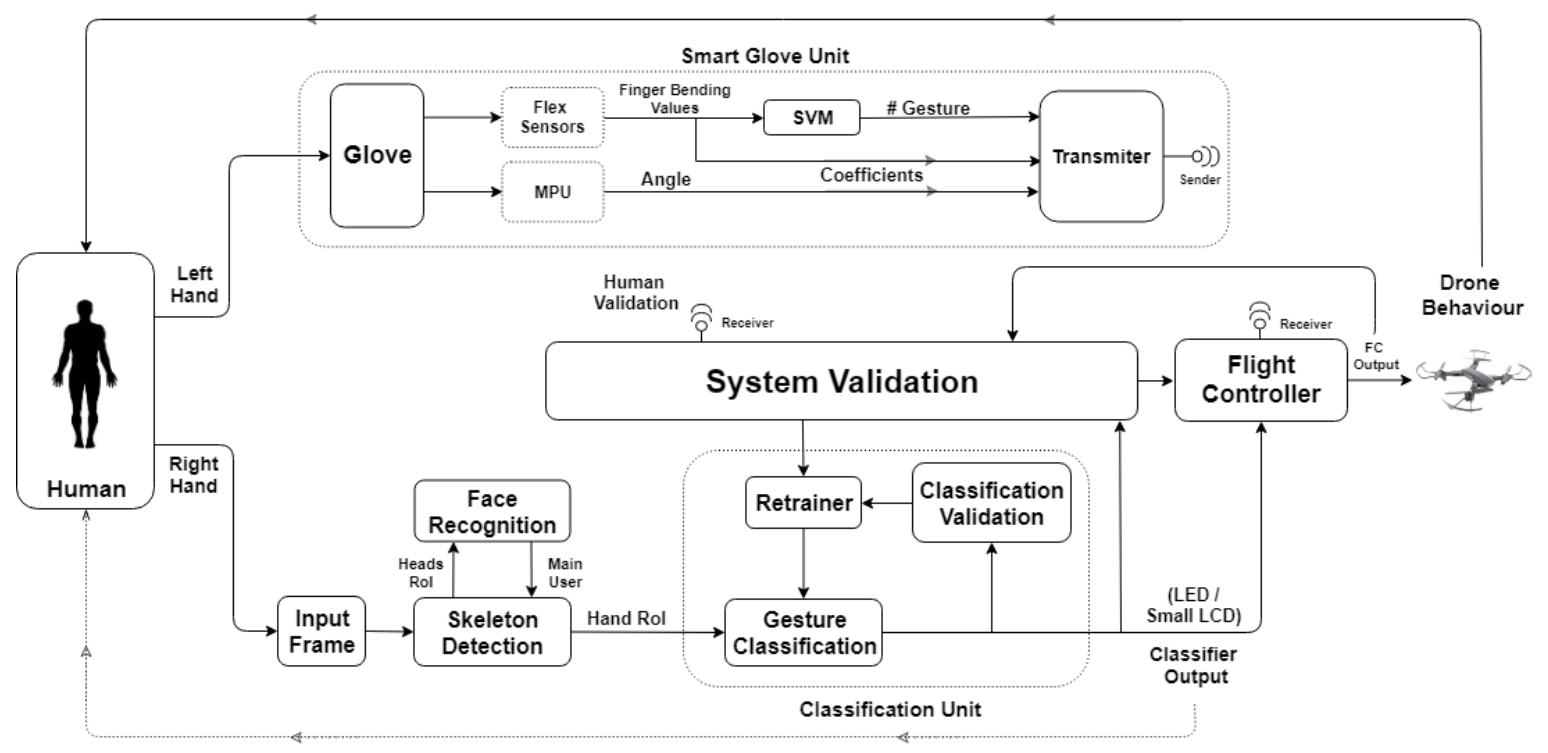

Figure 4.10: System block diagram for a multi-subject environment including three separate validation modules: (i) Classification Validation, (ii) System Validation, and (iii) human-in-the-loop validation.

Validation. In both single- and multi-subject environments, two types of gesture recognition are used to interact with an aerial vehicle: the smart glove-based method and the image processing-based method.

The Hand Gesture Classification unit in a multi-subject environment contains all the modules in the single-subject environment except for the Hand Detection module, as shown in Figures 4.11 and 4.12. The primary input to the Hand Gesture Classification unit is a

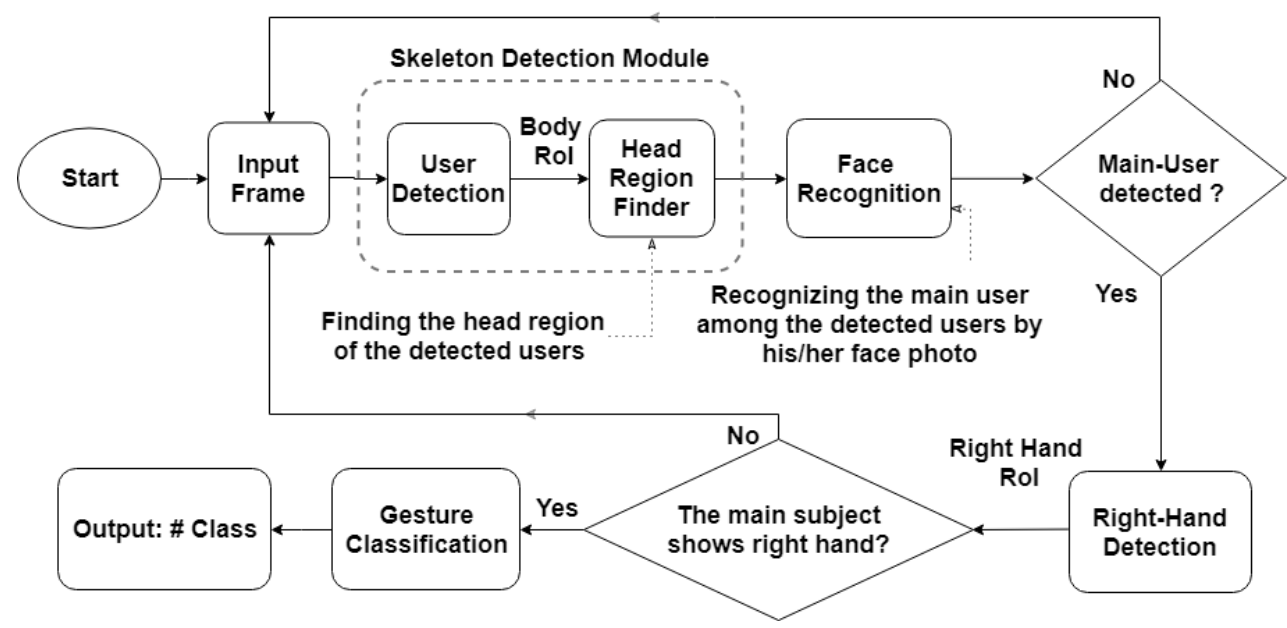

Figure 4.11: Flowchart of hand gesture recognition in a multi-subject environment. 


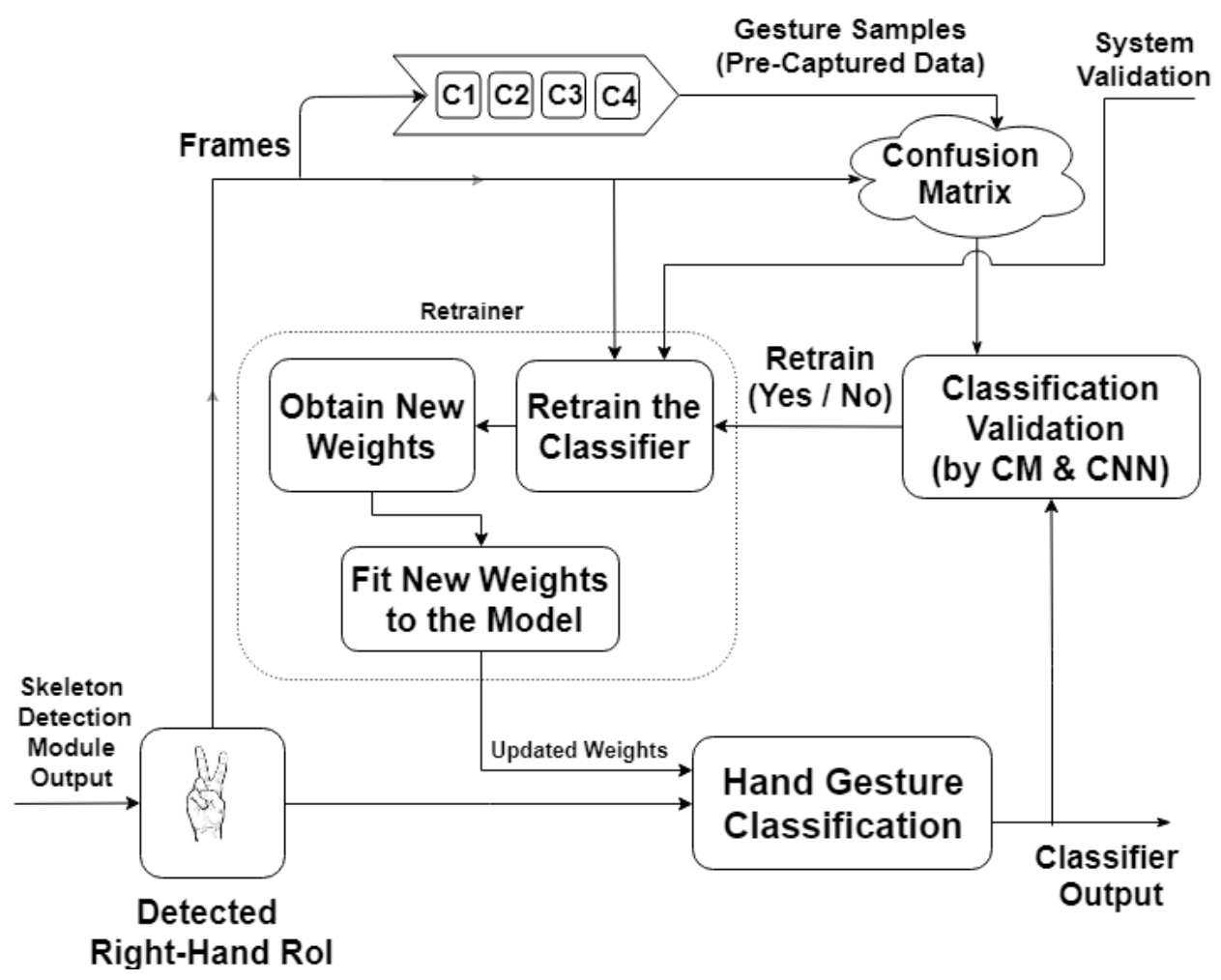

Figure 4.12: Gesture Classification block diagram in a multi-subject environment.

right-hand RoI, and the main output is the classified gesture represented as a class. In both single- and multi-subject environment cases, the classifier has an internal validation module that checks and improves the classifier's performance.

\subsubsection{Skeleton Detection Module}

In a multi-subject-environment scenario, the Skeleton Detection module recognizes the joints or each subject's skeleton in each input frame from the camera [41]. In order for the Skeleton Detection module to recognize joints of each subject, we assume that there is enough separation between the subjects. The proposed method is a top-down approach to detecting joints. The preliminary step to find each subject in every frame is to find a nose. The Skeleton Detection module begins from the nose, and then it searches for eyes, ears, a neck, and then the rest of the body as long as it is visible in the frame. If the Skeleton Detection module finds the nose and ears, the module counts that as a subject. Afterwards, 


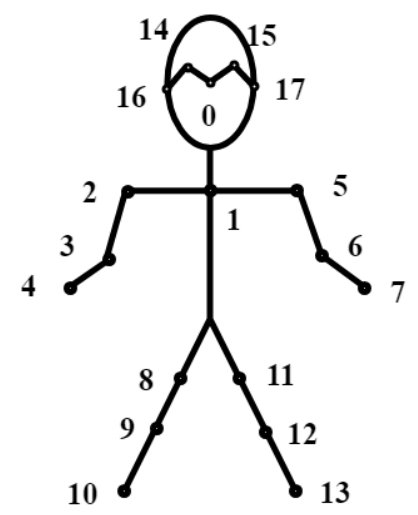

Figure 4.13: Numbered joints for the skeleton algorithm. The initial keypoint is the nose. Numbered joints for the skeleton algorithm [42]. The initial keypoint is the nose.

the module connects the detected joints and forms a representation of the body of each subject.

If the skeleton detector fails to detect all the joints of a subject (e.g., the subject is too close to the camera), the module saves the related coordinates of each joint and creates a numbered list of detected joints and their coordinates from the input frame. Figure 4.13 shows the pattern used to number the joints [42].

\subsubsection{Facial Recognition Module}

After the Skeleton Detection module detects subjects in each frame, the next step is to determine which subject is the main subject. Head regions detected by the Skeleton Detection module are fed to the facial recognition model to identify the main subject. If the facial recognition model confirms the existence of the main subject, the algorithm uses the joint coordinates obtained from the Skeleton Detection module to find the right hand of the main subject. For the facial recognition model, we use a condition-free (meaning no illumination changes or occlusions) facial recognition library with $99.38 \%$ accuracy that requires just one sample image to be trained [43]. This facial recognition model uses the histogram of oriented gradients (HOG) method to find the face patterns in the detected head region and the face landmark detection model to recognize the main subject's face $[44,45]$.

The goal of the HOG method is to find a pattern in the image that has a face. The HOG 

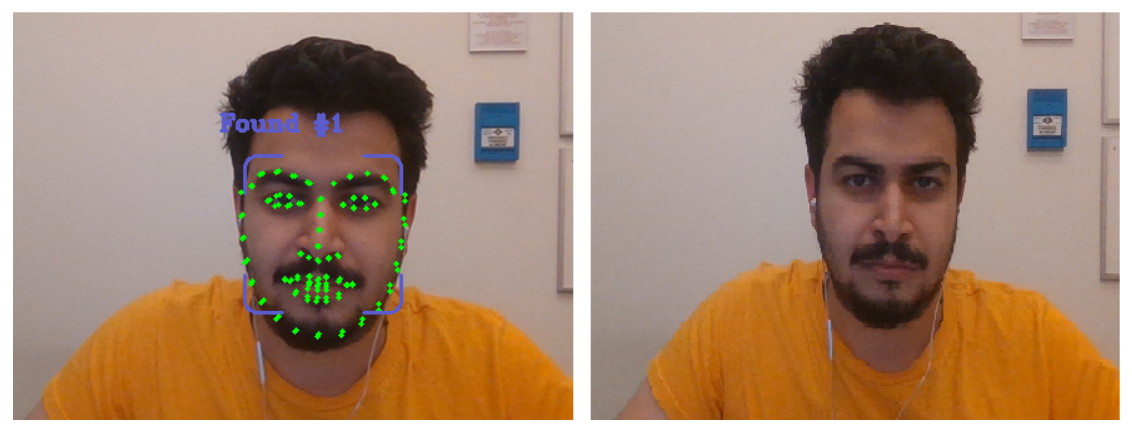

Figure 4.14: Face landmark detection.

finds the basic structure of the image based on the lighting of each pixel. The next step is to find the part of the image that is similar to a known HOG pattern extracted from a dataset of the main face [44].

The HOG model is pre-trained, while the face landmark detection model is unique for each person [45]. Figure 4.14 shows that the facial recognition model creates a face landmark for the main subject before the Skeleton Detection module begins processing. The facial recognition model then creates the landmark based on the single image of the main subject's face and uses it to detect this face among the detected head regions from the Skeleton Detection module. The proposed method requires a face image of the main subject to train the facial recognition model during the initialization phase.

\subsubsection{Right-Hand Detection Module}

The right-hand detection module, which is part of the Skeleton Detection module, searches through the detected joints of the main subject for the wrist of the right hand. The right-hand detection module then sends the right-hand region to the posture classification module (Figure 4.11). Chapter 4.5 describes how we evaluate the behavior of the algorithm in various multi-subject-environment scenarios, including with a hidden right hand and no gesture performed by the main subject. 


\subsubsection{Right-Hand Classifier}

Upon receiving the right-hand RoI as an input image, a CNN model classifies the detected hand into one of the predefined classes (Figure 4.9). In both cases, a single-subject and a multi-subject environment, the classifier output maps to one of the predetermined commands: take Off, land, turn right, or turn left. Subsequently, as illustrated in Figures 4.10 and 4.12, the output command is sent to the Classification Validation module to check the classification confidences and then to the next module to execute the command.

\subsubsection{Retrainer Module}

Figure 4.12 illustrates that the Retrainer module in both single- and multi-subject environments is activated in two cases: (i) when the Classification Validation module detects low confidence in the confusion matrix and (ii) when the System Validation module sends the retraining penalty signal. After the Retrainer module is enabled, as described in the Section 4.3.3 and [13], the Retrainer receives $S$ new images ( $S=5$ in our implementation) from the user as its input. The Retrainer module then retrains the running CNN model with the newly gathered data to obtain new weights. The module then fits the new weights to the CNN model and sends the CNN model to the classifier. The validation processes for a crowded environment are explained in Algorithm 2.

\subsection{Validation Modules}

Figures 4.7 and 4.10 show that the proposed algorithm for both single- and multi-subject environments has three validation modules that work together to improve system performance and reliability. In this section first we describe the human-in-the-loop validation, where the user is the first to validate the system output by observing the robot's behavior, second, we describe the outer layer of the system where there is a validation module that finds the source of a problem with either the classifier or the Flight Controller, and finally, 


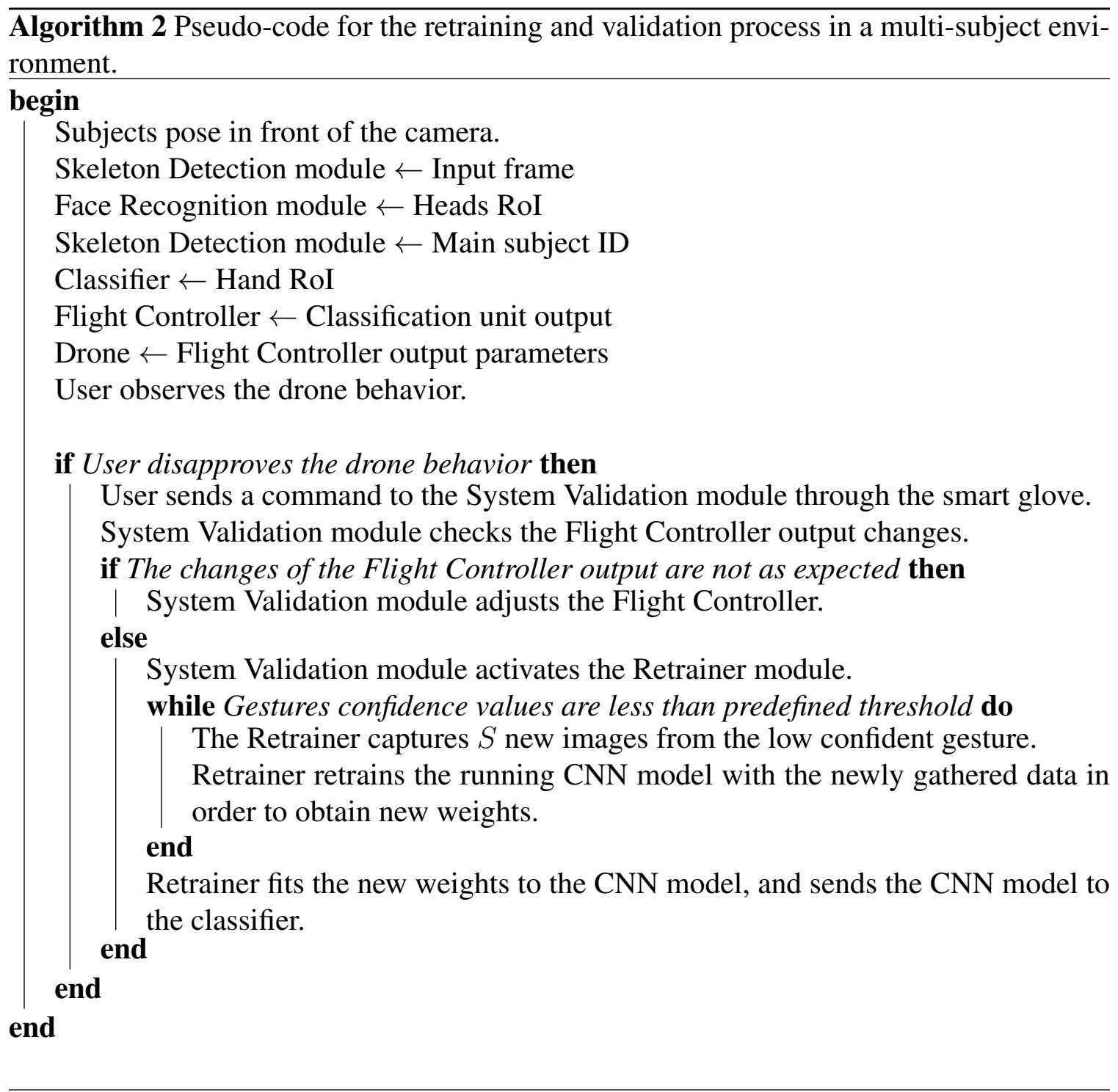

we describe the Classification Validation module which validates the gesture classifier output.

\subsubsection{Human-in-the-Loop Validation}

The user is responsible for two tasks: (i) to feed the system with hand gestures and (ii) to validate the drone's performance, as illustrated in Figure 4.7. By observing the drone's behavior, the user decides whether the system output is correct or not. If the user decides the output is incorrect, the user can indicate disapproval through the smart glove by 
performing Gesture \#15. The Gesture \#15 is set to declare an observed misbehavior and activate System Validation. Then, the System Validation checks both the classifier output and the Flight Controller output signals, to find the source of the observed misbehavior.

\subsubsection{Gesture Recognition Validation}

The Classification Validation module is responsible for checking the classifier's accuracy and, if necessary, to send a retraining command to the Retrainer. The Classification Validation module has access to the CNN model. A trustworthy source is required to test the reliability of the classifier output. We use $d$ images taken from the user during an initialization phase, and the system assigned them to the corresponding classes $(d=10$ in our implementation and experimental results). The Classification Validation module assumes that such data are correctly classified. The Classification Validation module then uses the running $\mathrm{CNN}$ model to create the confusion matrix (CM), as shown in Table 4.3, and analyzes the $\mathrm{CM}$ to calculate running $\mathrm{CNN}$ classification errors.

Table 4.3: Confusion matrix for the CNN model. Class \#1: take off, Class \#2: land, Class \#3: turn left, and Class \#4: turn right.

\begin{tabular}{c|c|c|c|c} 
- & Class \#1 & Class \#2 & Class \#3 & Class \#4 \\
\hline Class \#1 & $x_{1,1}$ & $x_{1,2}$ & $x_{1,3}$ & $x_{1,4}$ \\
\hline Class \#2 & $x_{2,1}$ & $x_{2,2}$ & $x_{2,3}$ & $x_{2,4}$ \\
\hline Class \#3 & $x_{3,1}$ & $x_{3,2}$ & $x_{3,3}$ & $x_{3,4}$ \\
\hline Class \#4 & $x_{4,4}$ & $x_{4,4}$ & $x_{4,4}$ & $x_{4,4}$
\end{tabular}

Let us define a classifier output reliability $R_{C_{i}}$ as

$$
R_{C_{i}}=\frac{x_{i, i}}{\sum_{j=1}^{C} x_{i, j}},
$$

where $x_{i, j}$ is an element of the CM, with $i, j \in\{1,2,3,4\}$, and $x_{i, i}$ is a diagonal element of the CM. If the classifier output reliability is less than a predefined threshold (in our implementation the threshold is set to $70 \%$ as a high accuracy that the classifier still classifies 
correctly), the Retrainer module retrains the CNN model with new images and updates the CNN weights.

When the classifier Retrainer is activated, $S=5$ images from the gesture with the lowest confidence are taken by sending a specific command through the smart glove. After gathering the $S=5$ images, with the mentioned system configuration, it takes up to 4.2 seconds to retrain the classifier and to fit the updated weights to the running CNN model. After each step (gathering images, retraining based on gathered images, and fitting the updated weights to the running CNN model), if the confidences are less than the threshold, the Classification Validation module reactivates the Retrainer. Repeatedly, the Retrainer takes the images produced by the user's command, retrains based on those images, and fits the CNN model. The Classification Validation module continues reactivating the Retrainer until all the calculated confidences become more than the threshold.

\subsubsection{System Validation}

Triggered by human validation, the System Validation module checks the performance of the Flight Controller and the classifier output. The System Validation initially assumes that the classifier output is correct and assumes the problem is related to the Flight Controller. If the Flight Controller check is passed, the initial assumption is proven wrong, so the System Validation module triggers the classification Retrainer module. After the System Validation module detects which output is incorrect, it transmits a command to the corresponding Retrainer to improve the performance.

To test the first assumption, the System Validation module compares the controller output parameters with their previous values for each command. For instance, if the classifier output command is landing, the System Validation module must receive a command to reduce the altitude parameter in the Flight Controller output; otherwise, it is a mismatch. If there is a mismatch, the System Validation module transmits a penalty command to the controller. Otherwise, the System Validation module sends the penalty command to the classification module to activate the Retrainer and update the CNN model. 
Table 4.4: Configuration of the proposed CNN layers.

\begin{tabular}{|c|c|}
\hline Layers & Config \\
\hline \multirow{6}{*}{ Conv2D } & activation: linear \\
\hline & batch input shape: $70 \times 70 \times 1$ \\
\hline & kernel size : $3 \times 3$ \\
\hline & use bias: true \\
\hline & filters: 32 \\
\hline & $\begin{array}{l}\text { kernel initializer: } \\
\text { class name: Variance Scaling } \\
\text { distribution: uniform } \\
\text { mode: fan avg } \\
\text { scale: } 1.0\end{array}$ \\
\hline Activation & ReLU \\
\hline Max-Pooling 2D & pool size $2 \times 2$ \\
\hline \multirow{6}{*}{ Conv2D } & activation: linear \\
\hline & batch input shape: $70 \times 70 \times 1$ \\
\hline & kernel size : $3 \times 3$ \\
\hline & use bias: true \\
\hline & filters: 64 \\
\hline & $\begin{array}{l}\text { kernel initializer } \\
\text { class name: Variance Scaling } \\
\text { distribution: uniform } \\
\text { mode: fan avg } \\
\text { scale: } 1.0\end{array}$ \\
\hline Activation & ReLU \\
\hline Max-Pooling 2D & pool size $2 \times 2$ \\
\hline \multirow{6}{*}{ Conv2D } & activation: linear \\
\hline & batch input shape: $70 \times 70 \times 1$ \\
\hline & kernel size : $3 \times 3$ \\
\hline & use bias: true \\
\hline & filters: 64 \\
\hline & $\begin{array}{l}\text { kernel initializer } \\
\text { class name: Variance Scaling } \\
\text { distribution: uniform } \\
\text { mode: fan avg } \\
\text { scale: } 1.0\end{array}$ \\
\hline Activation & ReLU \\
\hline Max-Pooling 2D & pool size $2 \times 2$ \\
\hline Flatten & - \\
\hline \multirow{3}{*}{ Dense } & activation: linear \\
\hline & $\begin{array}{l}\text { kernel initializer: } \\
\text { class name: Variance Scaling } \\
\text { distribution: uniform } \\
\text { mode: fan avg } \\
\text { scale: } 1.0\end{array}$ \\
\hline & units: 500 \\
\hline Activation & ReLU \\
\hline Dropout & rate: 0.5 \\
\hline \multirow{3}{*}{ Dense } & activation: linear \\
\hline & $\begin{array}{l}\text { kernel initializer } \\
\text { class name: Variance Scaling } \\
\text { distribution: uniform } \\
\text { mode: fan avg } \\
\text { scale: } 1.0\end{array}$ \\
\hline & units: 500 \\
\hline Activation & ReLU \\
\hline Dropout & rate: 0.2 \\
\hline \multirow{3}{*}{ Dense } & activation: linear \\
\hline & $\begin{array}{l}\text { kernel initializer } \\
\text { class name: Variance Scaling } \\
\text { distribution: uniform } \\
\text { mode: fan avg } \\
\text { scale: } 1.0\end{array}$ \\
\hline & units: 4 \\
\hline Activation & Sigmoid \\
\hline
\end{tabular}




\section{Chapter 5}

\section{Validation and Verification}

\subsection{Introduction}

This chapter presents the training and testing details of each model in this thesis. To validate the proposed algorithm's performance, we perform different scenarios with different group sizes in front of the camera. There is one main subject in each group that performs different gestures in front of the camera. We study the behavior of the proposed algorithm in different desired and undesired scenarios. Finally, this chapter presents comprehensive experimental results that validate the proposed algorithm for several desired and undesired cases.

Additionally, a multimedia file is attached to this thesis. We use CoppeliaSim software to test the proposed algorithm and create this multimedia file. This multimedia file shows a drone that receives commands from two sources: (i) a camera for right-hand gesture classification and (ii) a smart glove for left-hand gesture classification.

\subsection{CoppeliaSim}

We use a CoppeliaSim simulation environment to emulate a real-life scenario of drone control, and the drone behavior in this environment is recorded in the attached multimedia file. We demonstrated a drone's behavior based on four commands from the image-based 


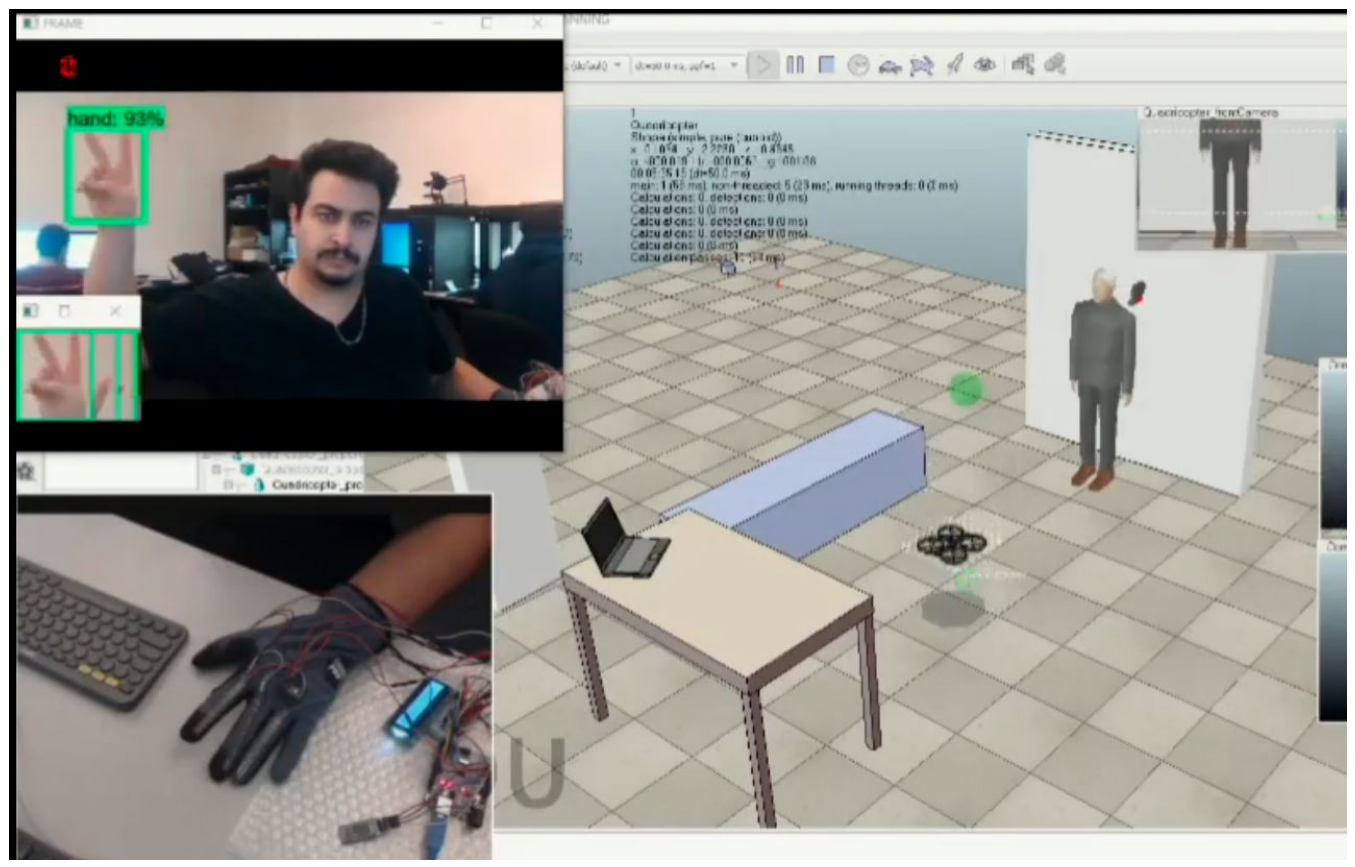

Figure 5.1: Multi-rotor aerial vehicle control using hand gestures and smart glove with selfvalidation.

input of the right-hand gesture and five commands from the smart glove-based input of the left-hand gesture (see Figure 5.1 and the multimedia file).

The multimedia file shows a drone that receives commands from two sources - a camera for the right-hand gesture classification, and a smart glove for left-hand gesture classification. A PID controller is applied as a Flight Controller for the drone. After the main subject performed the gestures through the smart glove and the camera, the proposed method creates and sends commands to the cloud-based environment. The Flight Controller reads the commands from the cloud-based environment and sets the drone controller parameters.

\subsection{Gesture Recognition Using Smart Glove}

The user calibrates the MPU by keeping his or her hand parallel to the ground and calibrates the flex sensors by fully closing and opening the fingers. The calibration steps and the smart glove's functionality are the same in both single- and multi-subject environments. Furthermore, the smart glove operates in two different modes simultaneously. 


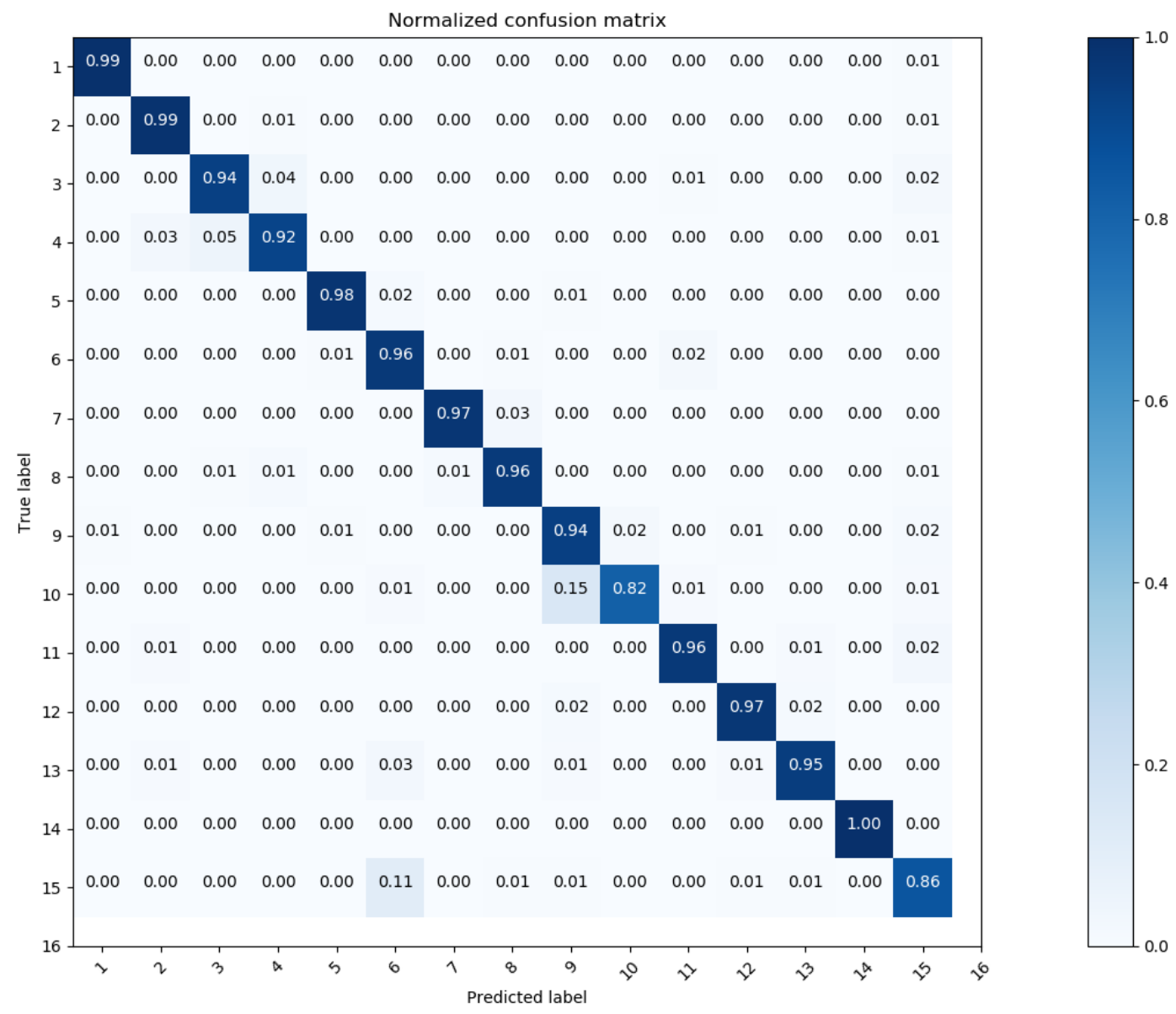

Figure 5.2: Support vector machine model confusion matrix for the left-hand gesture recognition.

The first mode considers each finger as an "on switch" when the finger is bent and an "off switch" when the finger is fully straight. The second mode uses the exact bending value from each flex sensor.

\subsubsection{Gesture Classifier Using the SVM Model}

The smart glove supports 16 different gestures and the SVM model with a linear kernel is used to analyze these gestures. This model is trained using 7216 samples on four fingers in 16 different classes (commands). Figure 5.2 demonstrates the CM of the SVM model after training.

Furthermore, Figure 5.3 shows the differences in value for each gesture. Descriptions of the gestures generated with the smart glove are provided in Table 4.1 and are the same 
in both single- and multi-subject environments.

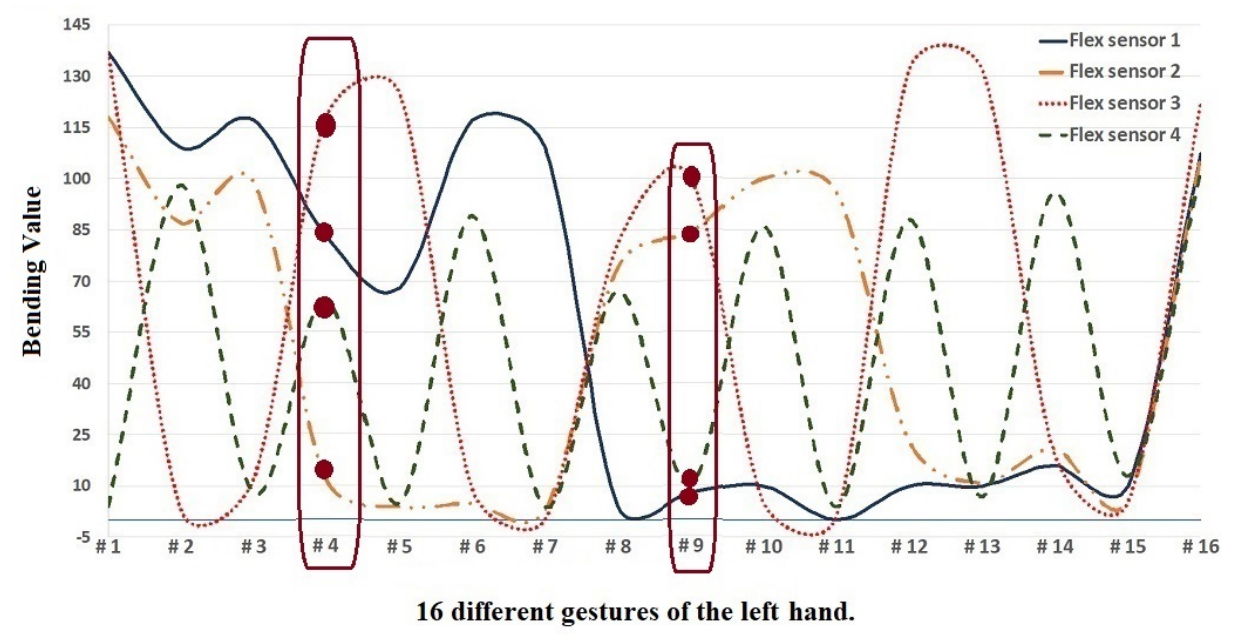

Figure 5.3: Flex sensors bending values through the 16 gestures of the smart glove. The generated signals with the flex sensors are used for left-hand gesture recognition and the generation of continuous control signals. As indicated on the horizontal axis, a combination of four bending values from the flex sensors represents a specific gesture.

\subsection{Gesture Recognition in a Single-Subject Environment}

Right-hand gesture recognition consists of a Hand Detection module and a Gesture Classification module. The algorithm detects the hand in each frame and then classifies the detected gesture. We implement the Hand Detection and the Gesture Recognition modules using the AUSDOM AW335 Full HD 1080p webcam. Moreover, we examine the performance of the validation system in various scenarios. A machine with 16 GB of RAM and NVIDIA Geforce $1060 \mathrm{Ti}$ GPU uses to train and validate the modules.

\subsubsection{Hand Detection Using the SSD Model}

From 46 different subjects, we obtain 4600 images (100 images per subject located at various distances from the camera) that are separated into training and validation sets. The training and validation sets consist of $85 \%$ and $15 \%$ of the total dataset, respectively. 
The loss function for the SSD model is a sum of the confidence loss $L_{\text {conf }}$ and localization losses $L_{l o c}$

$$
L(x, c, l, g)=\frac{1}{N}\left(L_{\text {conf }}(x, c)+L_{l o c}(x, l, g)\right)
$$

where $N$ is the number of matched default boxes. The confidence loss $L_{\text {conf }}$ is the softmax loss over multiple classes $c$ confidences and the localization loss $L_{l o c}$ is the loss between the predicted box $l$ and the ground truth box $g$ parameters [12].

Softmax function in the $L_{\text {conf }}$ is calculated as follows:

$$
L_{\text {conf }}(x, c)=\frac{e^{x}}{\sum_{j=1}^{c} e^{x_{j}}},
$$

where the $x$ is the input and the $e^{x}$ gives a positive value above 0 but not fixed in the range $(0,1)$, which is what is required of a probability. The $\sum_{j=1}^{c} e^{x_{j}}$ is the normalization term, which guarantees that all the output values of the function will sum to 1 , thus forming a valid probability distribution.

During training, the total loss between the training set and the validation set is expected to converge to the minimum. When the total loss converges to the minimum, an inference graph is generated, as shown in Figure 5.4. This graph compares the total loss to the training steps for the SSD. The tracking process is tested on a set of 635 new JPEG color images. The average accuracy is $94.28 \%$, which is obtained in 1.43 seconds.

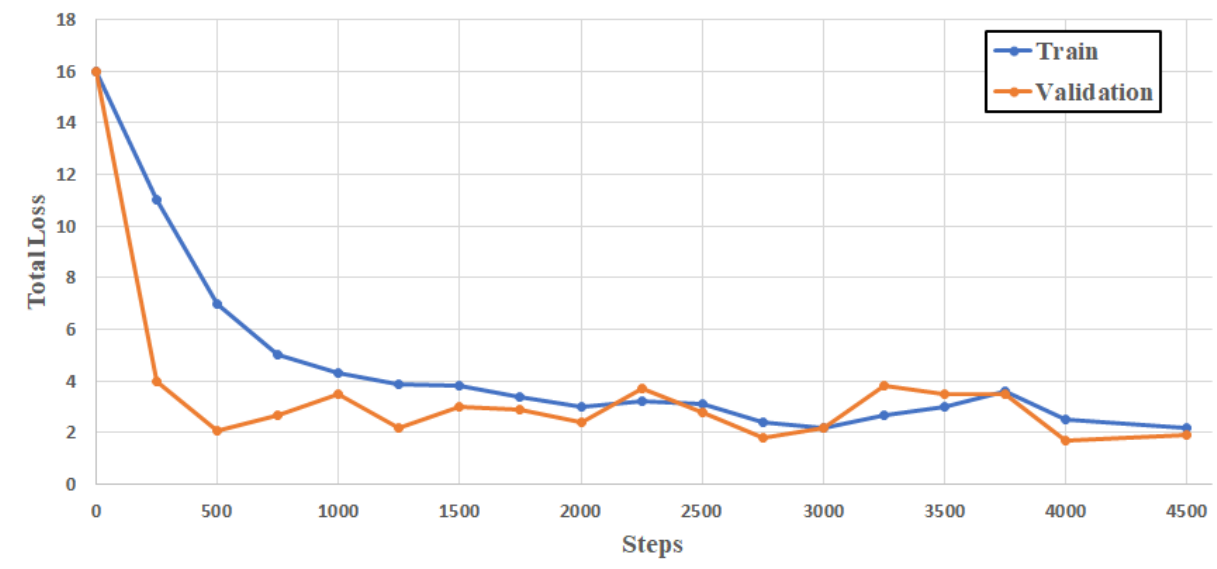

Figure 5.4: Total loss of the training process with respect to the steps. 


\subsubsection{Gesture Classifier Using the CNN Model}

A dataset is gathered by capturing 125 color images of size $640 \times 480$ pixels from each subject for each distance from the camera. In total, 5000 images are collected from 40 subjects standing at various distances from the camera (i.e., $1 \mathrm{~m}, 1.5 \mathrm{~m}, 2 \mathrm{~m}, 2.5 \mathrm{~m}$, and $3 \mathrm{~m})$. Subjects stand in front of a white background with their right hand showing four predetermined static gestures as shown in Figure 4.9. After manually filtering the blurry and noisy images, a dataset of 4600 JPEG color images is obtained.

To train the model, we divide the dataset into a training dataset and a testing dataset. We allocate $70 \%$ and $30 \%$ of all refined images to the training and testing sets, respectively. Data augmentation is used on the training set to prevent overfitting. A sign of an overfitted model is when the training loss is low and the validation loss is high [17]. In Figure 5.5(b), the training loss is higher than the validation loss. Both validation and training losses diminish until they reach their lowest value after 14 epochs and overfitting is avoided at each epoch.

The model is trained for 15 minutes for 15 epochs with an accuracy of $96.94 \%$. The batch size is 32, the Adam optimizer is chosen as the optimizer, and mean squared error is used as a loss function in the training process. After training, the system's accuracy on 120 images from each class (480 images the system had never seen before) is $98.12 \%$. Figure 5.5 shows the training and test graphs from this process.

Table 5.1 shows the accuracy, loss values, and their related training data sizes. The proposed CNN model, with details is given in Table 4.4, which shows convergence for data sizes larger than 700 images for each class (gesture). In addition, the data augmentation technique, based on vertical flipping and setting the degree range for random rotation, is used to reduce the required data size and reach a higher accuracy.

Note that the system performance in a single-subject environment in [13] is evaluated with various scenarios and penalties on the controller and the classifier. 


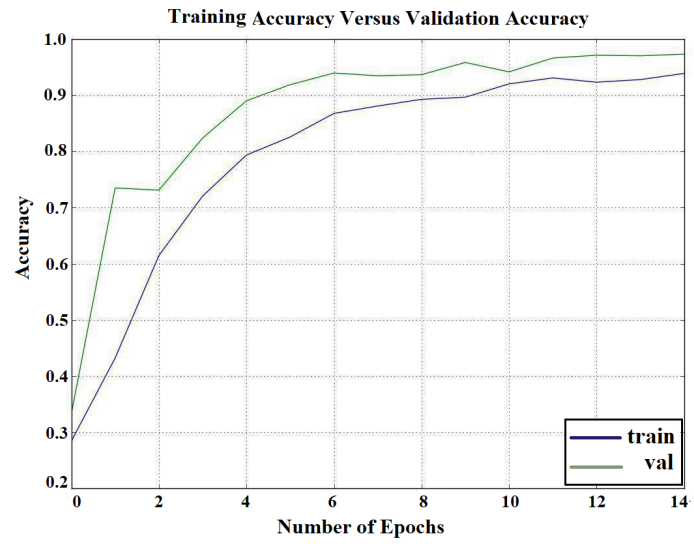

(a)

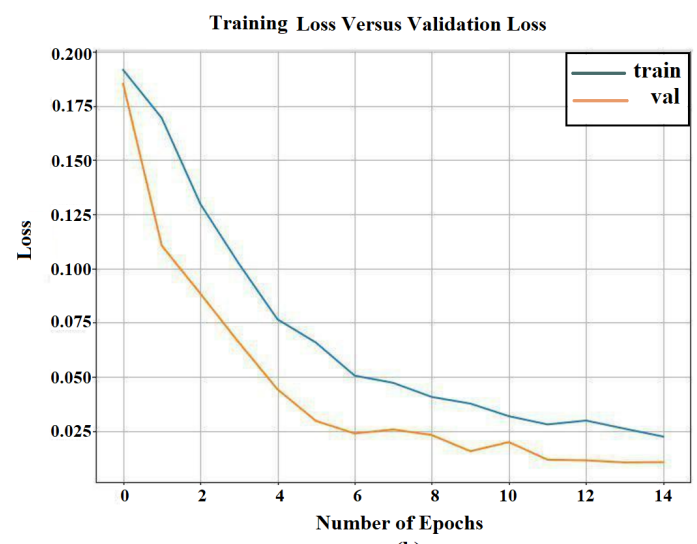

(b)

Figure 5.5: Training set versus validation set in a single-subject environment: (a) Accuracy; (b) Loss.

Table 5.1: Righ-hand gesture classifier training and validation results for different data sizes.

\begin{tabular}{|c|c|c|c|c|}
\hline $\begin{array}{c}\text { Data Size } \\
\text { (number of images } \\
\text { for each of four classes) }\end{array}$ & $\begin{array}{c}\text { Training } \\
\text { Accuracy } \\
\mathbf{\%}\end{array}$ & $\begin{array}{c}\text { Validation } \\
\text { Accuracy } \\
\mathbf{\%}\end{array}$ & $\begin{array}{c}\text { Train } \\
\text { Loss }\end{array}$ & $\begin{array}{c}\text { Validation } \\
\text { Loss }\end{array}$ \\
\hline 25 & 36.11 & 31.25 & 0.186 & 0.189 \\
\hline 50 & 50 & 46.77 & 0.159 & 0.151 \\
\hline 100 & 62.41 & 69.67 & 0.129 & 0.101 \\
\hline 200 & 82.03 & 81.4 & 0.074 & 0.068 \\
\hline 350 & 84.73 & 91.23 & 0.055 & 0.035 \\
\hline 500 & 89.59 & 95.18 & 0.039 & 0.019 \\
\hline $\mathbf{7 0 0}$ & $\mathbf{9 4 . 2 9}$ & $\mathbf{9 6 . 8}$ & $\mathbf{0 . 0 2 3}$ & $\mathbf{0 . 0 1}$ \\
\hline 1150 & 91.36 & 93.94 & 0.039 & 0.012 \\
\hline
\end{tabular}

\subsection{Gesture Recognition in a Multi-Subject Environment}

We demonstrate the system's performance for a multi-subject environment captured by a LOGI HD 1080p webcam. To implement the algorithm, we use an external Linux-based GPU and an NVIDIA Jetson AGX Xavier. The algorithm runtime for the multi-subject environment on the GPU for one and two subjects in the frame is 15 to 19 frames per second, and the runtime for three and four subjects in the frame is 10 to 13 frames per second.

To evaluate the accuracy and to study the behavior of the proposed algorithm in a multisubject environment, we created a dataset of subjects showing different predefined righthand gestures. We randomly selected $n$ subjects with different physical features and placed 
them in different group sizes to be photographed in various situations making predefined gestures in front of the camera. We distributed $n$ subjects in groups of $m$ users as shown in Table 5.2. After filtering and excluding blurry and noisy images, we use the remaining images to test the proposed method. Furthermore, we add another class in case the main subject does not perform any gestures and keep his or her right hand down. With each image, we test the proposed algorithm with different users based on the number of subjects in each image.

\subsubsection{Gesture Classifier Using the CNN Model}

To classify the right-hand gestures, the same CNN model is used in both single- and multi-subject environments. A dataset is created by capturing 125 color images of size $640 \times 480$ pixels for each user with varying distances from the camera. A total of 8125 images are collected from 65 subjects standing at various distances from the camera (i.e., $1 m, 1.5 m, 2 m, 2.5 m$, and $3 m$ ). After manually filtering the blurry and noisy images, a dataset of $7600 \mathrm{JPEG}$ color pictures is obtained. We use $70 \%$ of the refined images to train the $\mathrm{CNN}$ model for classification and use the rest as the test dataset.

To avoid overfitting at each epoch, the data augmentation technique is used in the training process, see Figure 5.6. The model is trained for 30 epochs with an accuracy of $97.33 \%$. The batch size is 32 , the Adam optimizer is chosen as the optimizer, and mean squared error is used as a loss function in the training process. After training, the system's accuracy on 300 images from each class (1200 images the system had never seen before) is $96.41 \%$.

Four right-hand gestures are used in the proposed algorithm's implementation for both single- and multi-subject environments; however, the proposed algorithm can handle a variety of numbers of gestures. For a different number of gestures, only the CNN model needs to be changed. The CNN model is described in Table 4.4, and the parameter that requires tuning in this case is the dense unit of the last layer. Afterward, the CNN model can receive training on $C$ gestures. In this thesis, four gestures $(C=4)$ are chosen, but there is no limitation on the number of gestures for the proposed algorithm. 


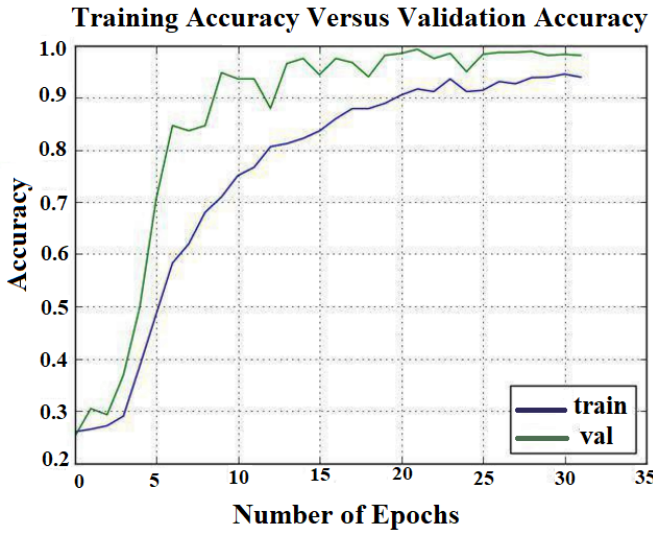

(a)

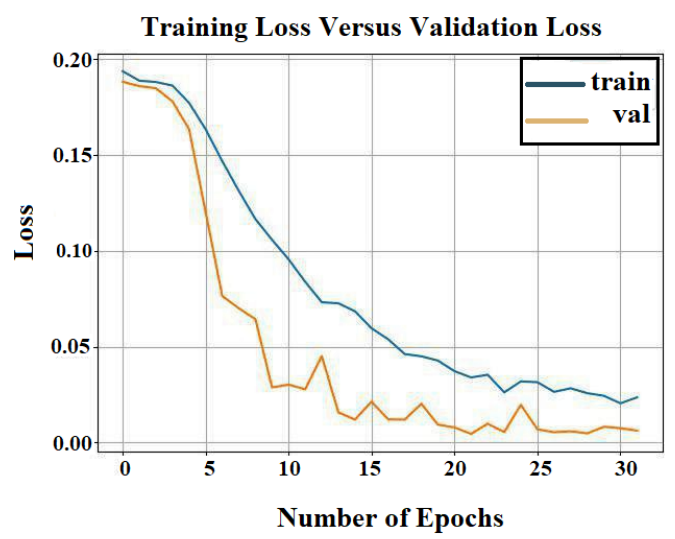

(b)

Figure 5.6: Training set versus validation set in a multi-subject environment: (a) Accuracy; (b) Loss.

\subsubsection{Skeleton Detection Using R-CNN Model}

To detect all subjects in the input frame, we use a joint-based detection module as the Skeleton Detection module [41]. We implement this joint-based detection module with an R-CNN network on the GPU with 15 frame rate per seccond (FPS). The proposed algorithm maps the joints, as shown in Figure 4.13.

The Skeleton Detection module has four primary responsibilities: (i) detecting the initial key points, which are the nose and ears of each subject in the input frame, (ii) detecting the joints of every subject in the input frame, (iii) obtaining the head RoI for every detected subject, and (iv) finding the main subject's right-hand RoI.

To test the proposed method in a multi-subject environment, we randomly organize subjects into 35 groups of a single subject, 190 groups of two subjects, 1120 groups of three subjects, and 1260 groups of four subjects. Each group is used to test all the main modules, such as Skeleton Detection, facial recognition, right-hand detection for the main subject, and right-hand gesture classification. Table 5.2 shows a summary of results for each of the groups of single, two, three, and four subjects. The proposed method is tested on 10420 images. 
Table 5.2: Gesture recognition in a multi-subject environment: Experimental results for four different group populations. A group of $m$ subjects refers to the population of subjects in the input frame which means there are $m$ subjects in the frame.

\begin{tabular}{|c|c|c|c|c|c|c|c|c|c|c|c|}
\hline \multirow{2}{*}{ Population } & \multirow{2}{*}{$\begin{array}{c}\text { Number } \\
\text { of } \\
\text { Subjects }\end{array}$} & Total & Detec & ion & Recogn & ition & $\begin{array}{l}\text { Right- } \\
\text { Detec }\end{array}$ & Iand & Gest & ation & $\begin{array}{l}\text { Algorithm } \\
\text { Accuracy }\end{array}$ \\
\hline & & Images & Correct & $\%$ & Correct & $\%$ & Correct & $\%$ & Correct & $\%$ & $\%$ \\
\hline 1 & 35 & 140 & 140 & 100 & 140 & 100 & 140 & 100 & 129 & 92.14 & 98.04 \\
\hline 2 & 20 & 760 & 756 & 99.47 & 755 & 99.87 & 755 & 100 & 679 & 89.93 & 97.32 \\
\hline 3 & 16 & 4,480 & 4,395 & 98.1 & 4,309 & 98.04 & 4,258 & 98.82 & 3,601 & 87.57 & 94.88 \\
\hline 4 & 10 & 5,040 & 4,809 & 95.42 & 4612 & 95.9 & 4471 & 96.94 & 3137 & 70.16 & 89.61 \\
\hline
\end{tabular}

\subsection{Effect of Activating a Flex Sensor on Velocity of the Drone Movement}

To study the performance of sensors, the third flex sensor $f_{3}$ is activated and the effect of this activated sensor is discussed. As presented in Table 5.3, the third flex sensor, $f_{3}$, senses bending after 1 second, and the output of the SVM module therefore changes from 0000 to 0100 . Immediately after the change in the SVM module output, the proposed algorithm begins to calculate the change. The computed change for the third flex sensor, $r s_{3}(t)$, rises from 0 to 31.32 , and consequently, the $V_{G}(t)$ increases by $0.74 m / s$. The $V_{G}(t)$ changes until the SVM module output resets to 0000, and later, the $r s_{3}(t)$ decreases back to zero or its minimum. The $V_{G}(t)$ variation and bending fluctuation for the third flex sensor are demonstrated in Figure 5.7. In Figure 5.7, the left vertical axis presents the bending value of the flex sensors, and the right vertical axis shows the velocity. 
Table 5.3: Effect of the activated flex sensor on the drone velocity. Note: $f_{n}$ : bending value of the $n$-th flex sensor, $G \# m$ : $m$-th gesture with the smart glove, $V_{G}(t)$ : drone velocity.

\begin{tabular}{|l|l|l|l|l||l||l|l|}
\hline Time & $f_{1}$ & $f_{2}$ & $f_{3}$ & $f_{4}$ & SVM output & $r s_{3}(t)$ & $V_{G}(t)$ \\
\hline \hline 1 & 10.67 & 15.21 & 5.78 & 0.37 & $G \# 0: 0000$ & 0 & $V_{0}=1$ \\
\hline 3 & 12.19 & 7.82 & 68.41 & 15.72 & $G \# 2: 0 \underline{100}$ & 38.41 & 1.2671 \\
\hline 5 & 15.82 & 11.65 & 56.98 & 7.98 & $G \# 2: 0 \underline{100}$ & 26.98 & 1.1834 \\
\hline 7 & 3.49 & 0.03 & 118.71 & 13.19 & $G \# 2: 0 \underline{100}$ & 88.71 & 1.6032 \\
\hline 9 & 9.34 & 2.42 & 101.82 & 11.73 & $G \# 2: 0 \underline{100}$ & 71.82 & 1.4883 \\
\hline 11 & 18.31 & 9.98 & 95.36 & 20.41 & $G \# 2: 0 \underline{100}$ & 65.36 & 1.4444 \\
\hline 13 & 15.19 & 9.06 & 31.93 & 11.88 & $G \# 0: 0000$ & 0 & $V_{0}=1$ \\
\hline
\end{tabular}

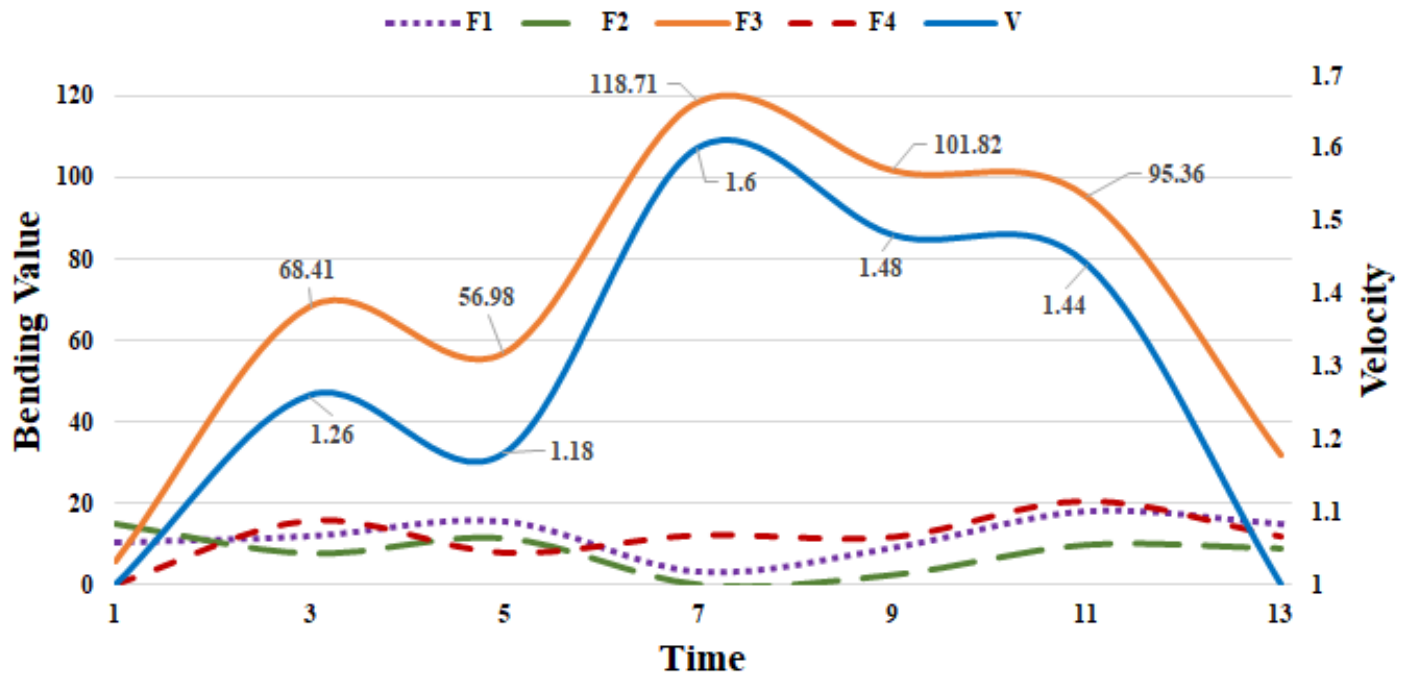

Figure 5.7: Activating the third flex sensor by bending the third flex sensor $\left(f_{3}\right)$ and its effect on the velocity of the drone.

\subsection{Test Scenarios}

We tested various scenarios to study the system's behavior as follow:

- Testing validation modules in three different scenarios:

- Validation system with no penalty.

○ Penalty on the Flight Controller.

- Penalty on the classifier.

- Testing the system performance in different single- and multi-subject environments: 
$\circ$ Frame contains both hands.

$\circ$ Frame without right hand.

○ Frame contains right hand but there is no posture.

○ Frame contains correct hand.

First, we tested the validation modules as presented in Figure 5.8. In all scenarios, the user shows Gesture \#1 (a command to take off), but in each scenario, the user observes a different response.

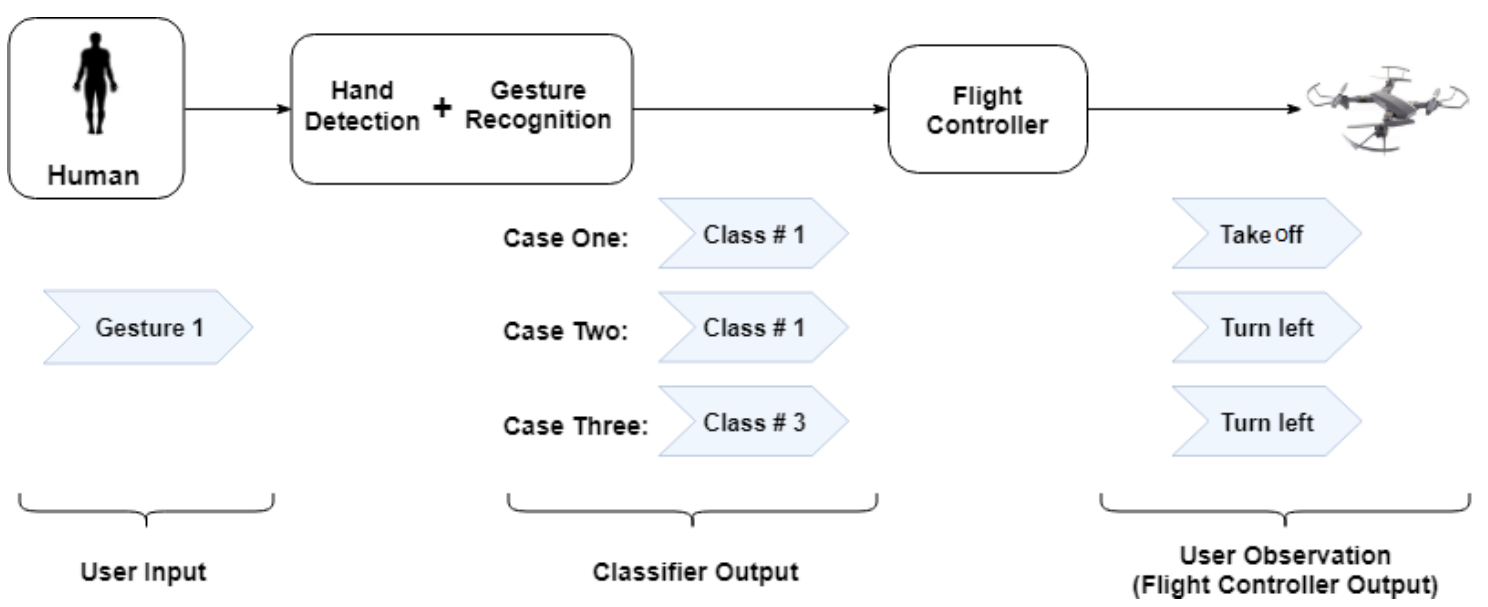

Figure 5.8: Three cases for the System Validation: 1) No penalty, 2) Penalty on the Flight Controller, and 3) Penalty on the classifier. The commands are: Class \#1: take off, Class \#2: land, Class \#3: turn left, and Class \#4: turn right.

\subsubsection{Scenario 1: System Validation With No Penalty}

In Scenario 1, the drone is on the ground, and the user makes Gesture \#1, as shown in Figure 5.9. The Hand Detection module obtains the hand RoI and sends it to the Gesture Classification module. Hence, the classifier classifies Class \#1. The classifier output is sent to the Flight Controller, and the Flight Controller generates a take off command. The user observes the drone taking off. Therefore, the user confirms the output (drone behavior). In this scenario, the system operates correctly. 


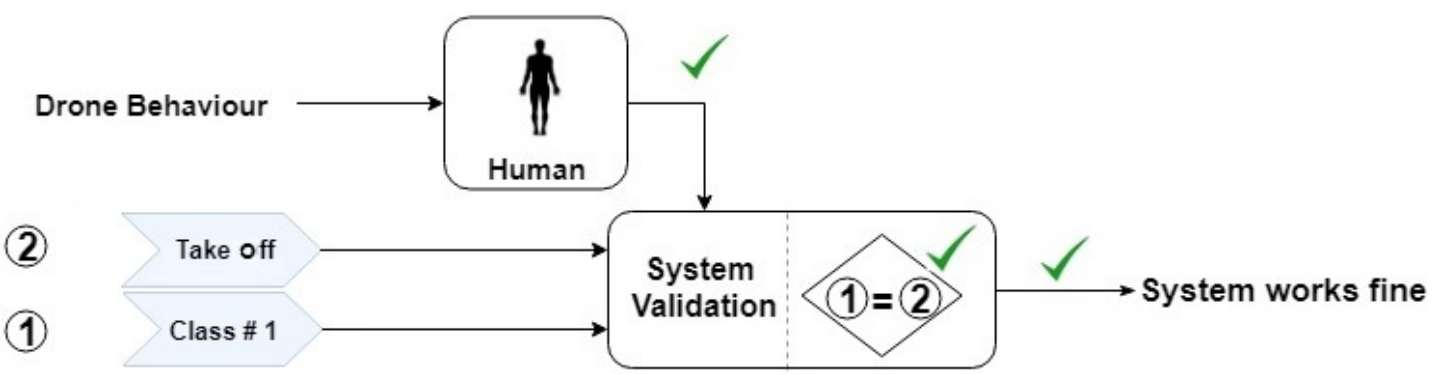

Figure 5.9: System without penalty. The commands are: Class \#1: take off, Class \#2: land, Class \#3: turn left, and Class \#4: turn right.

\subsubsection{Scenario 2: Penalty on the Flight Controller}

For the second scenario, the drone is on the ground again, and the user shows Gesture $\# 1$, as shown in Figure 5.10. As in the previous scenario, the classifier produces Class \#1 as its output. The classifier output is sent to the Flight Controller, and this time, an error causes the Flight Controller to generate the turn left command instead of take off. Hence, the user rejects the drone behavior and sends a command to the System Validation module through his or her smart glove. When the System Validation module receives Class \#1 from the classifier, expecting an increase in the Flight Controller output values, it then understands the mismatch and determines that the problem is with the Flight Controller. The System Validation module sends a penalty command to the Flight Controller, improving its future performance.

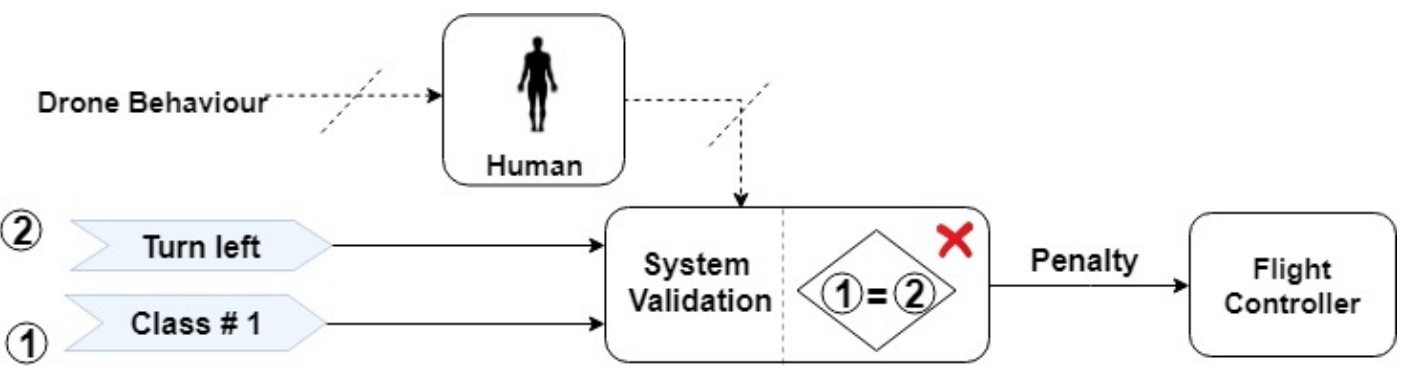

Figure 5.10: Penalty signal for the controller. The commands are: Class \#1: take off, Class \#2: land, Class \#3: turn left, and Class \#4: turn right. 


\subsubsection{Scenario 3: Penalty on the Classifier}

The user shows Gesture \#1, as shown in Figure 5.11. The hand detector chooses the hand RoI and transfers the RoI to the classifier. This time, the classifier provides Class \#3 instead of Class \#1 and then sends the classifier output to the Flight Controller. The Flight Controller presents the turn left command and forwards the command to the drone. The user observes the drone turning left but had expected the drone to take off. Therefore, the user rejects the drone behavior using the smart glove.

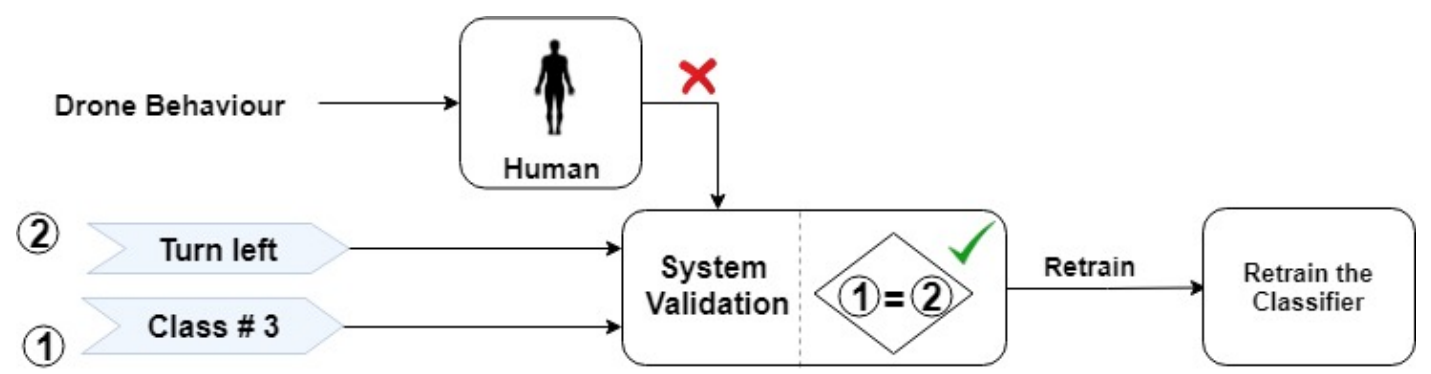

Figure 5.11: Penalty signal for the classifier. The commands are: Class \#1: take off, Class \#2: land, Class \#3: turn left, and Class \#4: turn right.

As indicated in Table 5.4 and Table 5.5, the System Validation module sends this penalty to the classifier. The classifier is then retrained in three steps. In the first step, the System Validation module checks the states of the classifier output and the controller output. The System Validation module discovers the controller operation is acceptable, so the problem is with the classifier. Next, the System Validation module sends the penalty

Table 5.4: Penalty on the classifier: Class \#4. Classification Validation decides to retrain the model on Class \#4 in three steps to improves the confidence from $54.55 \%$ to $79.19 \%$. The four classes are: Class \#1: take off, Class \#2: land, Class \#3: turn left, and Class \#4: turn right.

\begin{tabular}{|c||c|c|c|c||c|c|c|c||c|c|c|c|}
\hline \multicolumn{1}{|c||}{ Steps } & \multicolumn{4}{c||}{ Step \#1 } & \multicolumn{4}{c||}{ Step \#2 } & \multicolumn{4}{c|}{ Step \#3 } \\
\hline \hline Class & 1 & 2 & 3 & 4 & 1 & 2 & 3 & 4 & 1 & 2 & 3 & 4 \\
\hline 1 & 8 & 3 & 0 & 0 & 11 & 0 & 0 & 0 & 11 & 0 & 0 & 0 \\
2 & 0 & 6 & 0 & 0 & 0 & 6 & 0 & 0 & 0 & 5 & 1 & 0 \\
3 & 0 & 1 & 10 & 0 & 1 & 0 & 10 & 0 & 0 & 0 & 11 & 0 \\
4 & 0 & 5 & 0 & 6 & 5 & 0 & 0 & 11 & 5 & 0 & 0 & 16 \\
\hline \hline Confidant \% & 72.73 & 100 & 90.91 & 54.55 & 100 & 100 & 90.91 & 68.73 & 100 & 83.33 & 100 & 79.19 \\
\hline
\end{tabular}


Table 5.5: Penalty on the classifier: Class \#2. Classification Validation decides to retrain the model on Class \#2 in three steps to improves the confidence from $16.67 \%$ to $100 \%$. The four classes are: Class \#1: take off, Class \#2: land, Class \#3: turn left, and Class \#4: turn right.

\begin{tabular}{|c||c|c|c|c||c|c|c|c||c|c|c|c|}
\hline \multicolumn{1}{|c||}{ Steps } & \multicolumn{4}{c||}{ Step \#1 } & \multicolumn{4}{c||}{ Step \#2 } & \multicolumn{4}{c|}{ Step \#3 } \\
\hline \hline Class & 1 & 2 & 3 & 4 & 1 & 2 & 3 & 4 & 1 & 2 & 3 & 4 \\
\hline 1 & 5 & 0 & 0 & 1 & 5 & 1 & 0 & 0 & 5 & 1 & 0 & 0 \\
2 & 0 & 1 & 5 & 0 & 0 & 11 & 0 & 0 & 0 & 11 & 0 & 0 \\
3 & 0 & 0 & 7 & 0 & 0 & 0 & 7 & 0 & 0 & 0 & 7 & 0 \\
4 & 0 & 0 & 1 & 7 & 0 & 2 & 1 & 5 & 0 & 0 & 0 & 11 \\
\hline \hline Confidant \% & 83.33 & 16.67 & 100 & 87.50 & 83.33 & 100 & 100 & 62.50 & 83.33 & 100 & 100 & 100 \\
\hline
\end{tabular}

command to the classification module. The Retrainer then retrains the classifier and obtains new weights.

As presented in Table 5.4 and Table 5.5, when the classifier Retrainer is activated, $S=5$ images from the gesture with the lowest confidence are taken by sending a specific command through the smart glove. After gathering the $S=5$ images, with the mentioned system configuration, it takes around 4.2 seconds to retrain the classifier and to fit new weights to the running $\mathrm{CNN}$ model. After each step (gathering images, retraining based on gathered images, and fitting the new weights to the running CNN model), if the confidences are less than the threshold, the Classification Validation module reactivates the Retrainer. Repeatedly, the Retrainer receives the images taken from the user's hand, retrains based on those images, and fits the CNN model. The Classification Validation module continues reactivating the Retrainer until all the calculated confidences become more than the threshold.

In cases such as Scenario 2 and Scenario 3, where the system behavior is not what the user had expected, the drone will be frozen by receiving Gesture \#15 through the smart glove. The drone will then take no commands unless the validation process is finished. 


\subsubsection{Scenario 4: Frame Contains Both Hands}

As shown in Figures 5.12a and 5.12b, the user shows both hands at the correct angles performing the correct gestures. In the first step, the proposed algorithm detects the user in the frame and the user's joints through the Skeleton Detection module. Then, the algorithm sends the user's head RoI to the facial recognition module to find the main subject. After the facial recognition module identifies the main subject, the Skeleton Detection module finds the right-hand RoI (the algorithm also works on the left hand by changing the interested joint from joint 4 to 7 ; see Figure 4.13) and sends it to the classification module.

\subsubsection{Scenario 5: Frame Without Right Hand}

The user makes a gesture with the left hand, hiding the right hand, Figure 5.12d. As the first step, the Skeleton Detection module detects the subject in the frame and sends the subject's head RoI to the facial recognition module and returns a confirmation that the main subject has been detected. Then, the Skeleton Detection module searches for joint 4 , and when it cannot find it in the list of the detected joints, the process ends until the user shows his or her right hand.

\subsubsection{Scenario 6: Frame Contains Right Hand but There Is No Pos- ture}

The user shows no gestures and keeps the right hand down, Figures 5.14b, 5.14c, 5.15a and 5.15c. The proposed algorithm detects the right-hand joint and gets ready to capture the hand region and sends it to the classification module. Before doing so, the algorithm calculates the angle between joints 2 and 4 (Figure 4.13). The correct angle between joints 2 and 4 for a gesture in front of the camera is between 0 to 90 degrees. Therefore, if the user opens the right hand further than this range, the algorithm assumes that the right hand points toward the ground and the result is no gesture. 


\subsubsection{Scenario 7: Frame Contains Correct Hand}

The subjects within the frame, including the main subject, show different gestures, and the proposed algorithm detects the main subject and his or her right-hand gestures.

Note: in the following examples the classifier output number starts from zero to three for four classes, but the algorithm output that we mentioned in this thesis starts from one to four for four classes. The mapping Table 5.6 is provided to clarify this.

Table 5.6: Mapping table between classifier output and the algorithm output.

\begin{tabular}{|c|c|}
\hline \multicolumn{2}{|c|}{ Class Numbers } \\
\hline $\begin{array}{c}\text { Classifier Output } \\
\text { (top left corner in the images) }\end{array}$ & $\begin{array}{c}\text { Algorithm Output/Class } \\
\text { (captions) }\end{array}$ \\
\hline 0 & 1 \\
\hline 1 & 2 \\
\hline 2 & 3 \\
\hline 3 & 4 \\
\hline
\end{tabular}




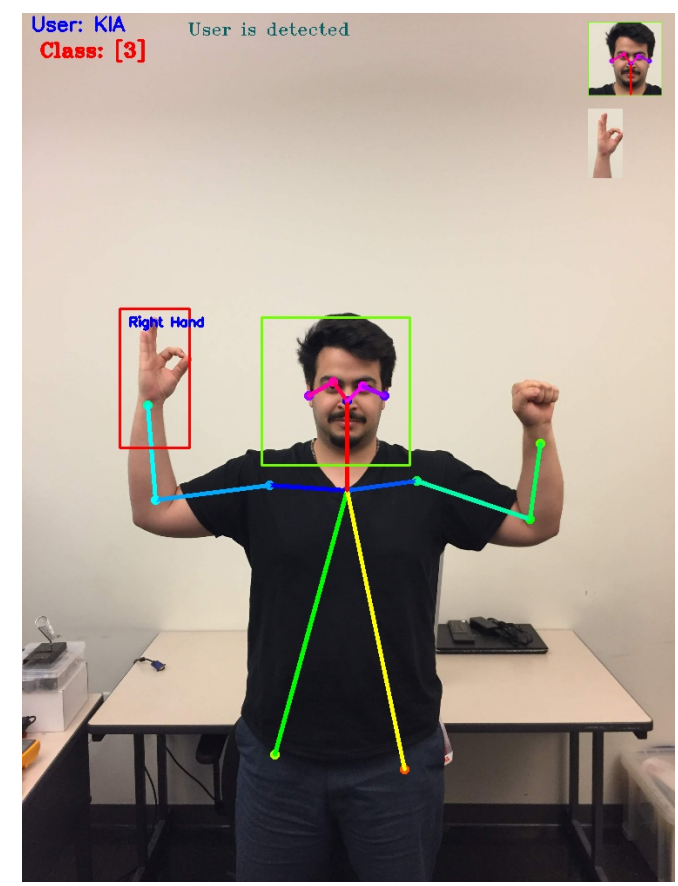

(a) Class \#4: turn right

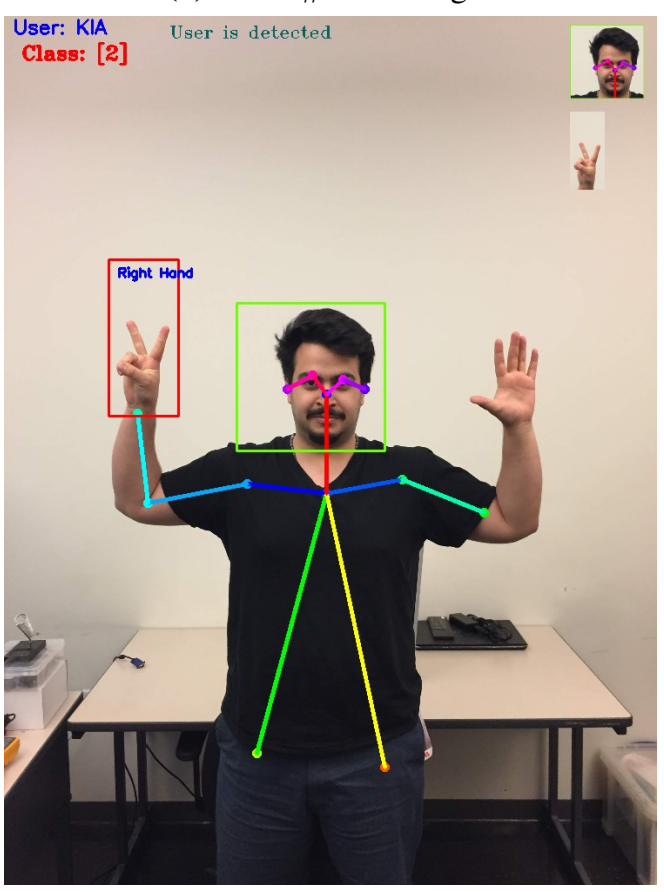

(c) Class \#3: turn left

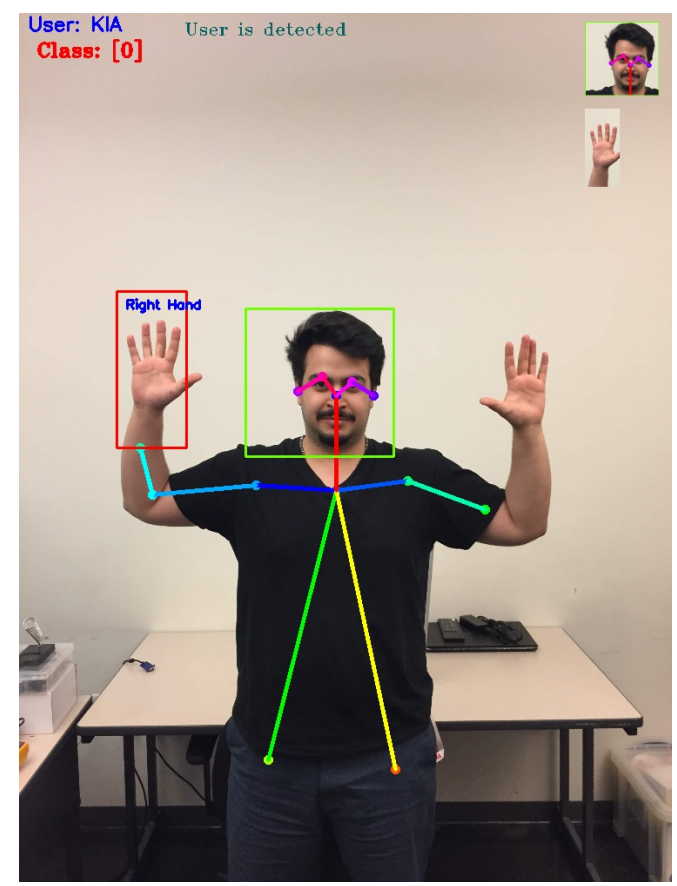

(b) Class \#1: take off

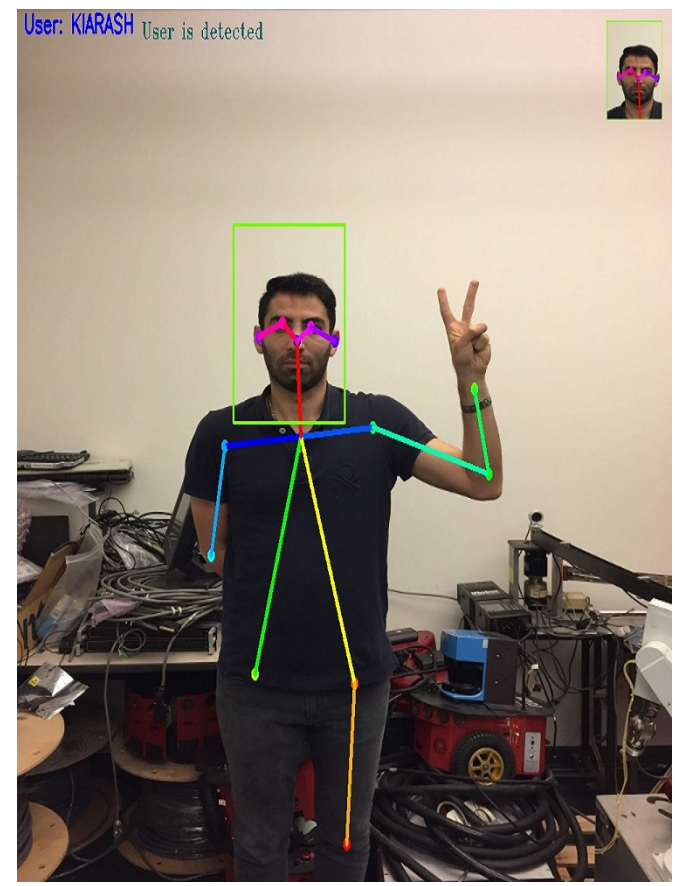

(d) No right hand

Figure 5.12: Gesture recognition on a single subject gesturing in front of the camera (series 1) . The commands are: Class \#1: take off, Class \#2: land, Class \#3: turn left, and Class \#4: turn right. 


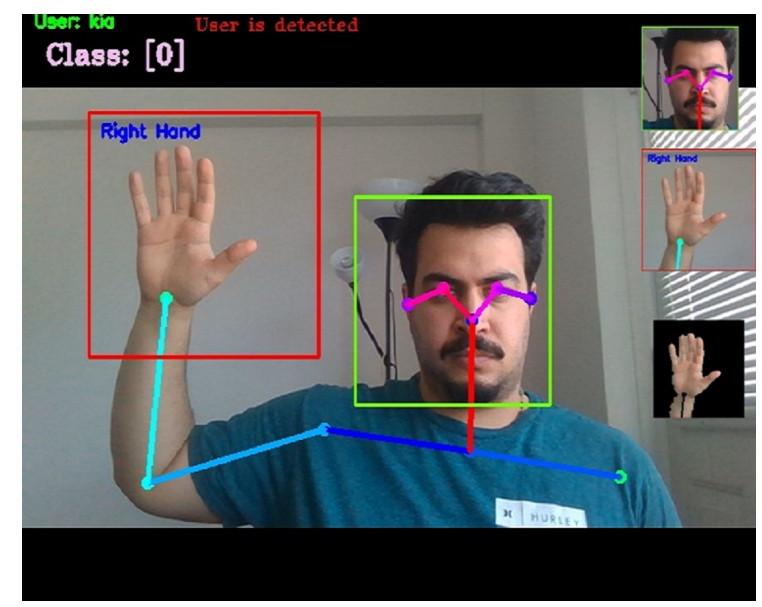

(a) Class \#1: take off

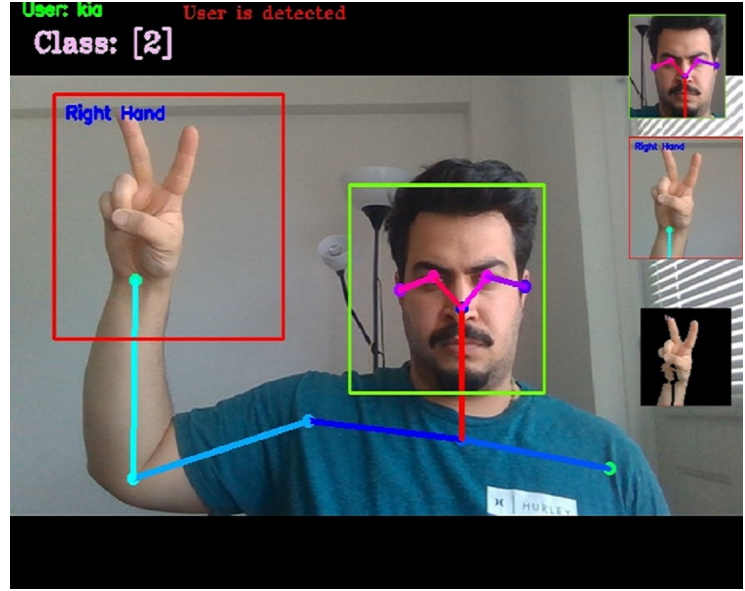

(b) Class \#3: turn left

\section{Class: [3]}

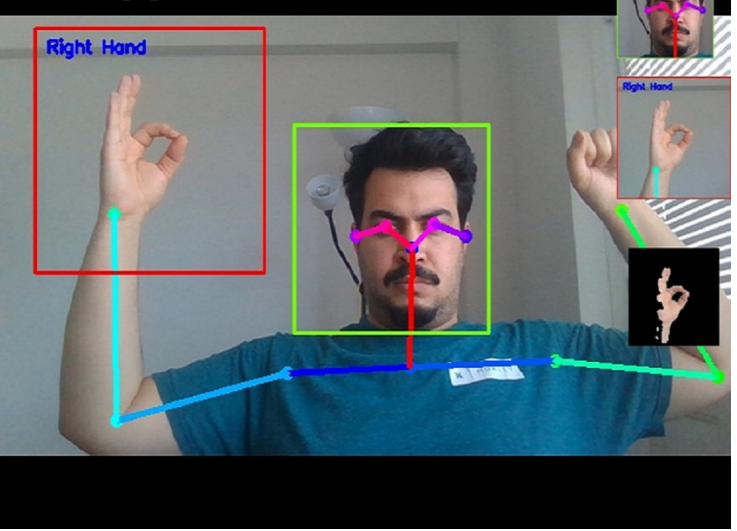

(c) Class \#4: turn right

Figure 5.13: Gesture recognition on a single subject gesturing in front of the camera (series 2). The commands are: Class \#1: take off, Class \#2: land, Class \#3: turn left, and Class \#4: turn right. 


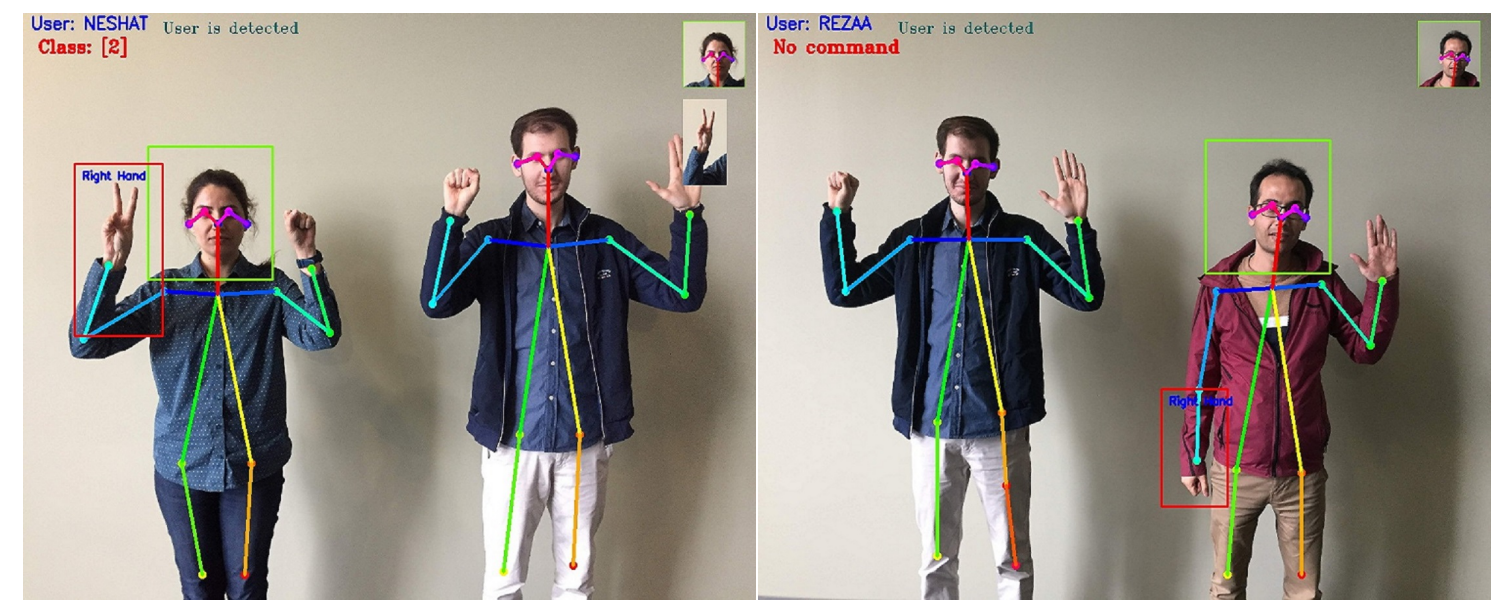

(a) Class \#3: turn left

(b) No posture

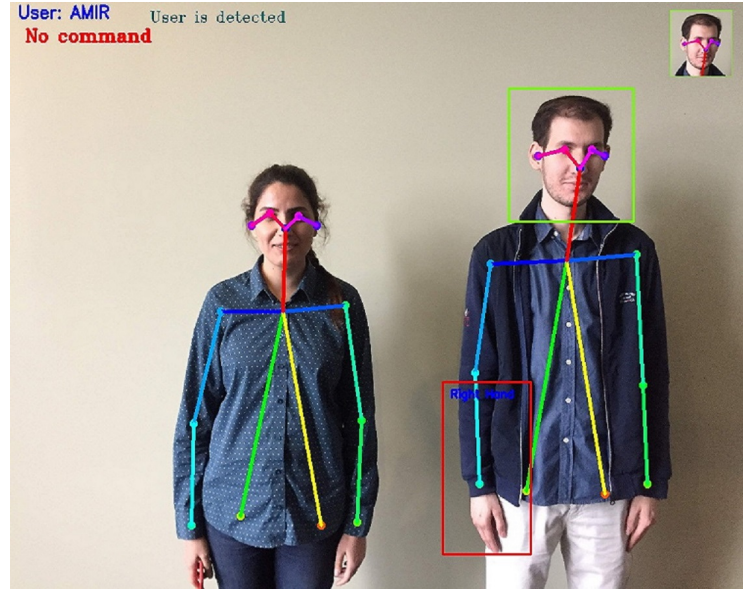

(c) No posture

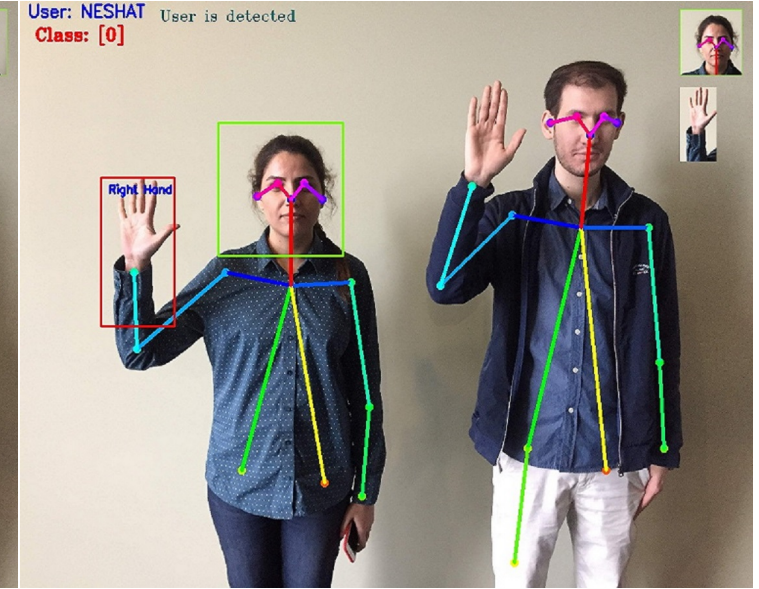

(d) Class \#1: take off

Figure 5.14: Gesture recognition on two (2) subjects including a main subject gesturing in front of the camera (series 1). 


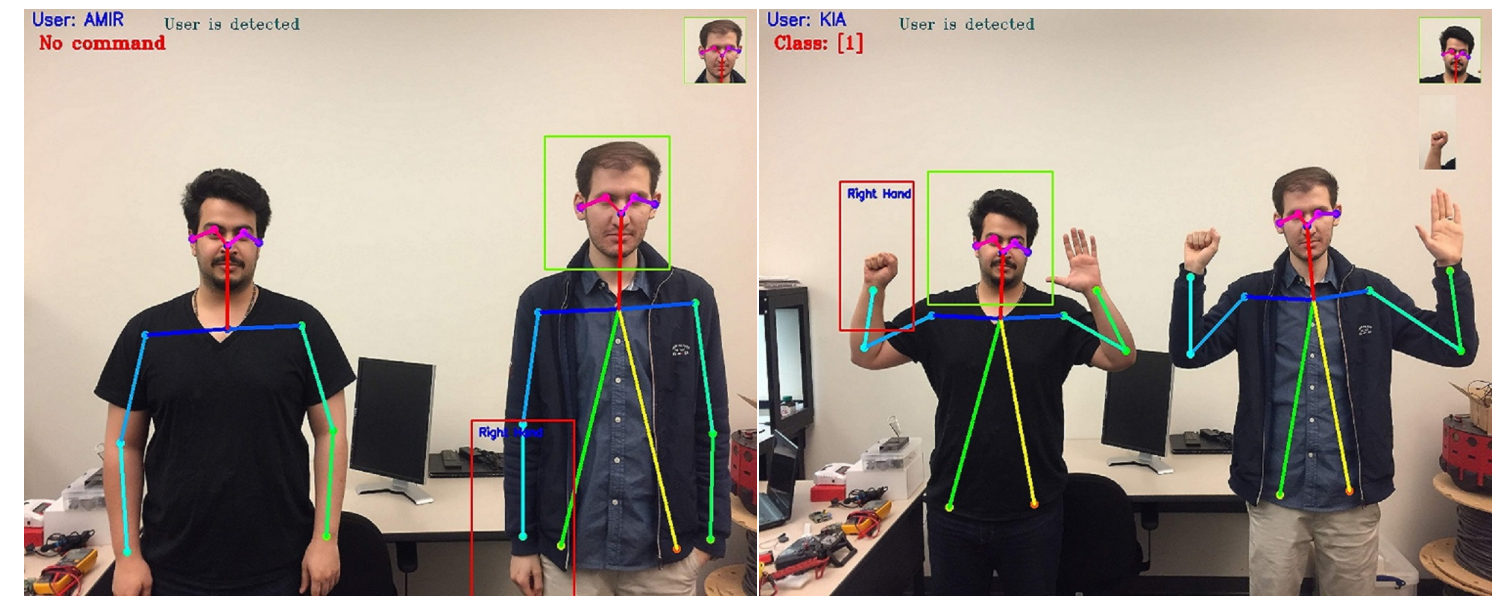

(a) No posture

(b) Class \#2: land

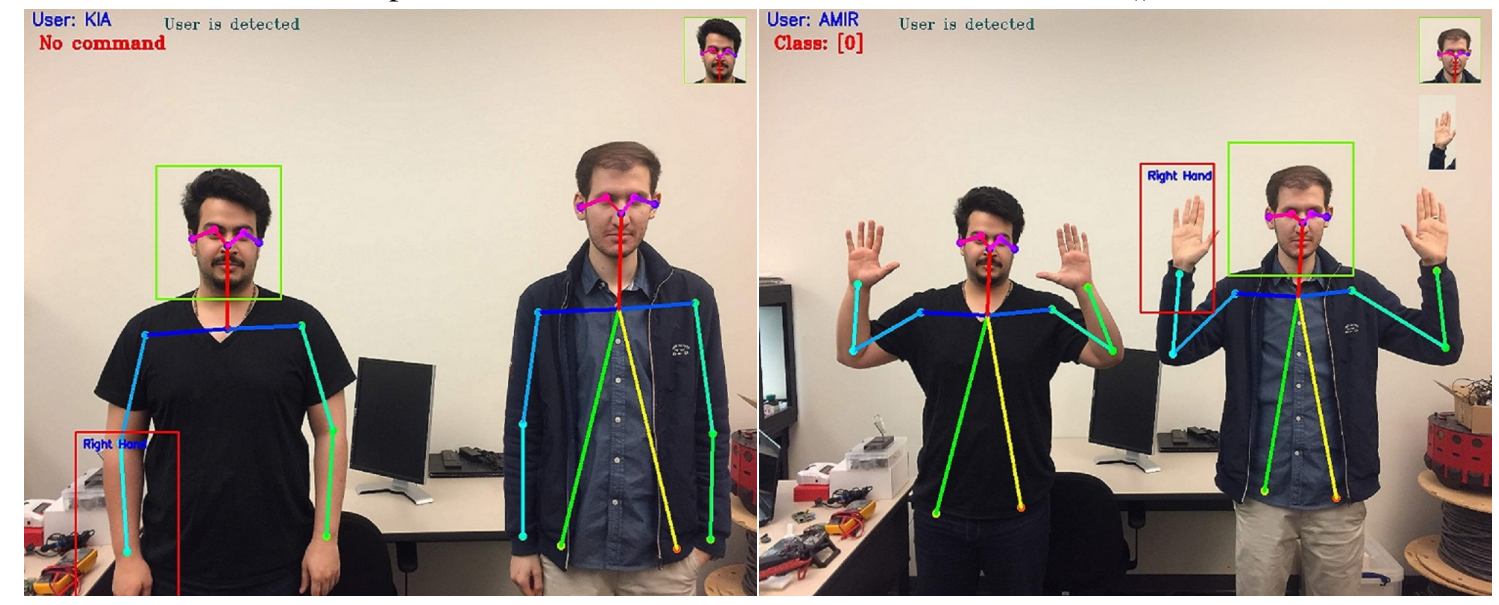

(c) No posture

(d) Class \#1: take off

Figure 5.15: Gesture recognition on two (2) subjects including a main subject gesturing in front of the camera (series 2). 


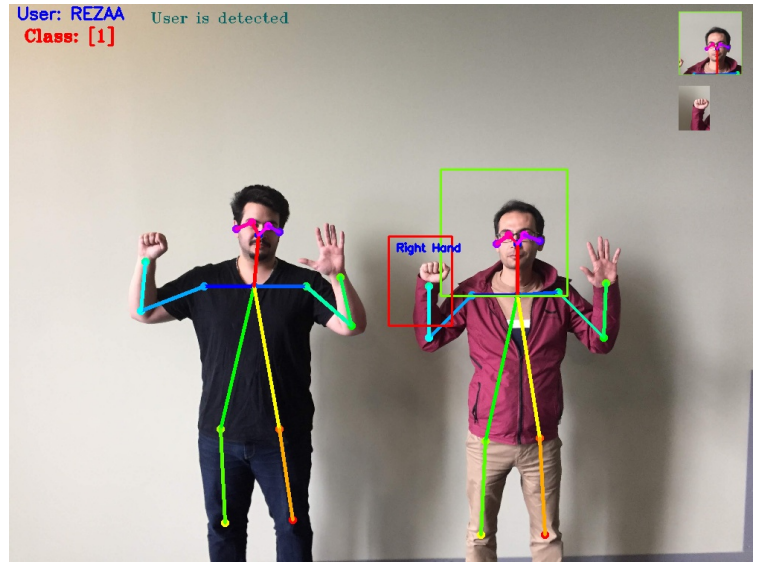

(a) Class \#2: land

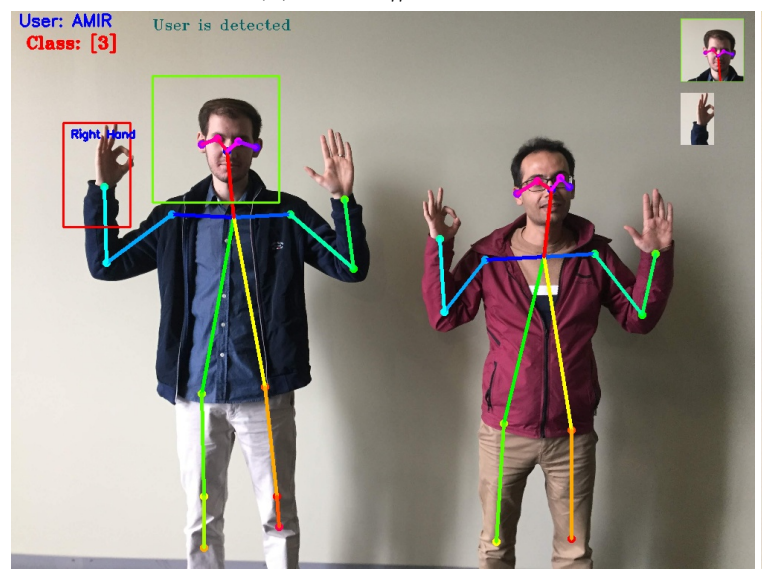

(c) Class \#4: turn right

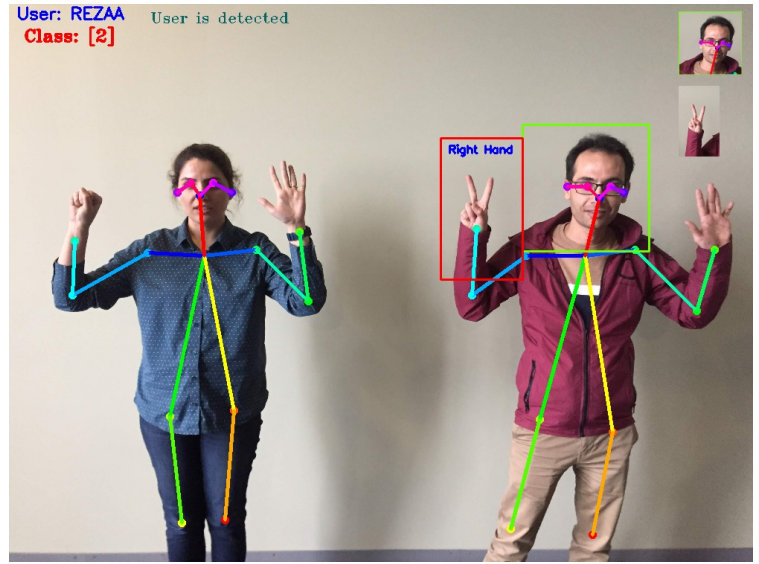

(e) Class \#3: turn left
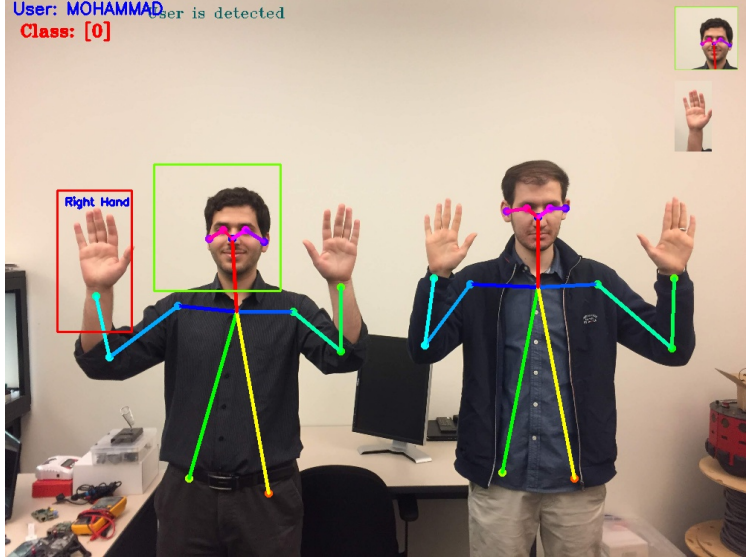

(b) Class \#1: take off

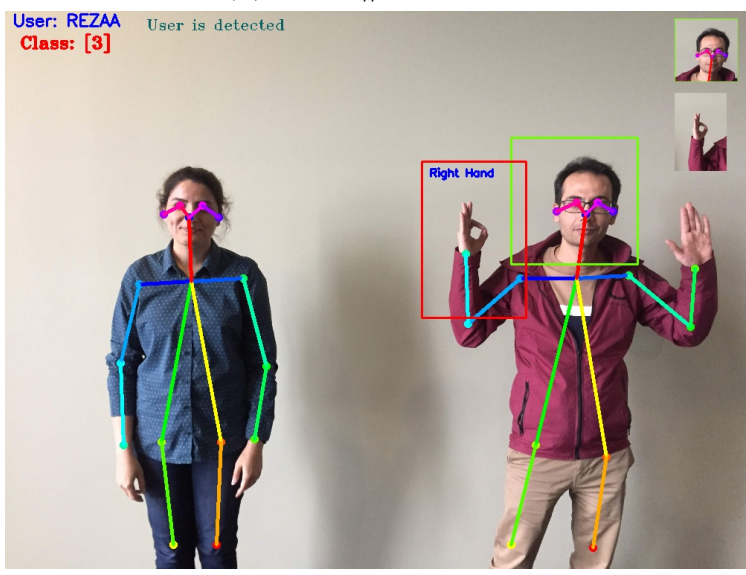

(d) Class \#4: turn right

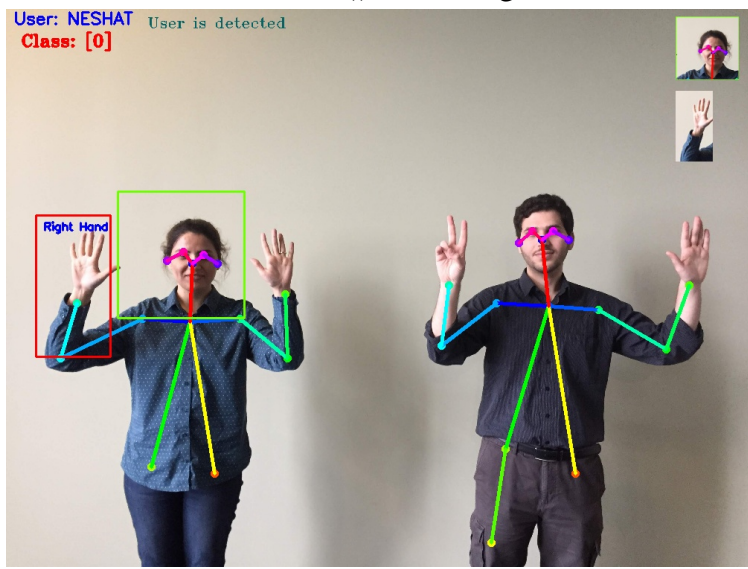

(f) Class \#1: take off

Figure 5.16: Gesture recognition on two (2) subjects including a main subject gesturing in front of the camera (series 3 ). 


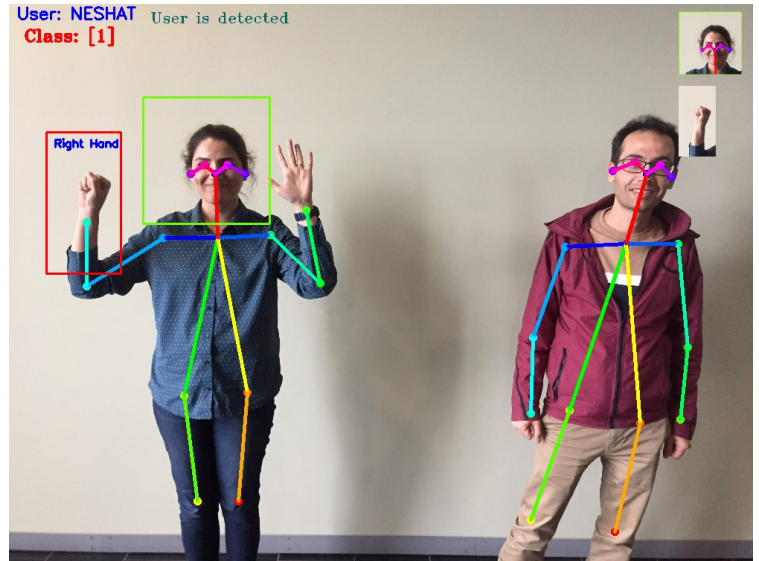

(a) Class \#2: land

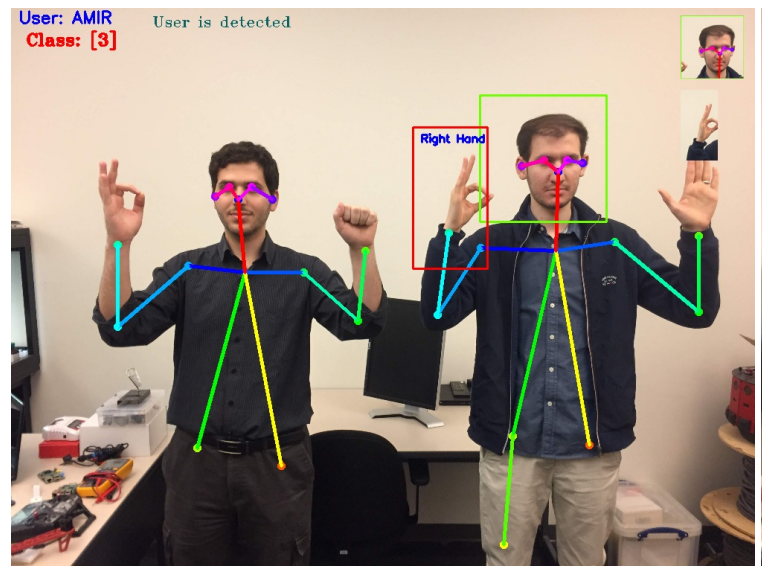

(c) Class \#4: turn right

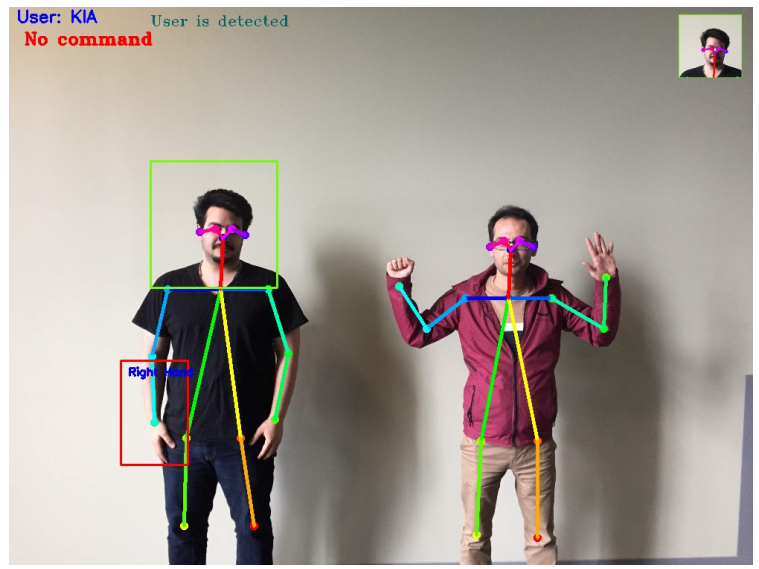

(e) No posture

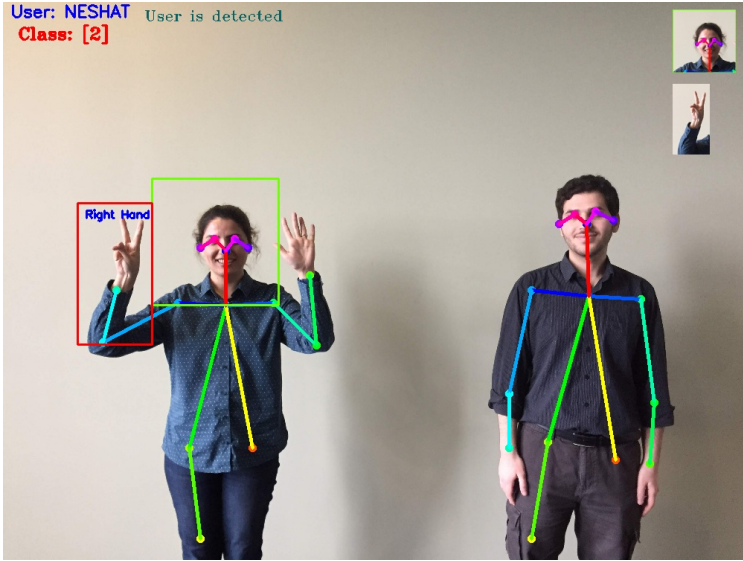

(b) Class \#3: turn left

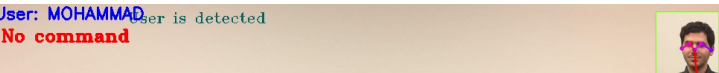

2

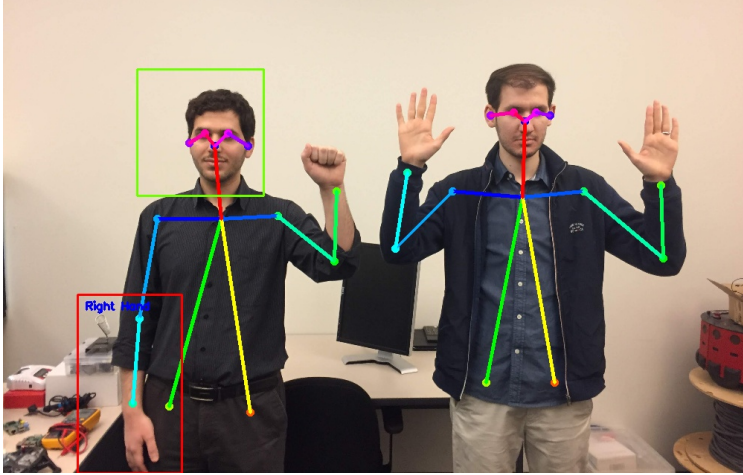

(d) No posture

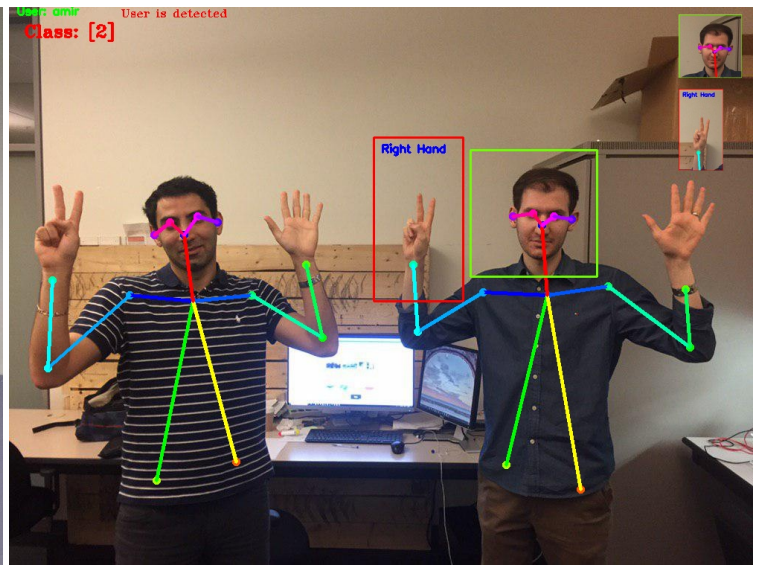

(f) Class \#3: turn left

Figure 5.17: Gesture recognition on two (2) subjects including a main subject gesturing in front of the camera (series 4). 


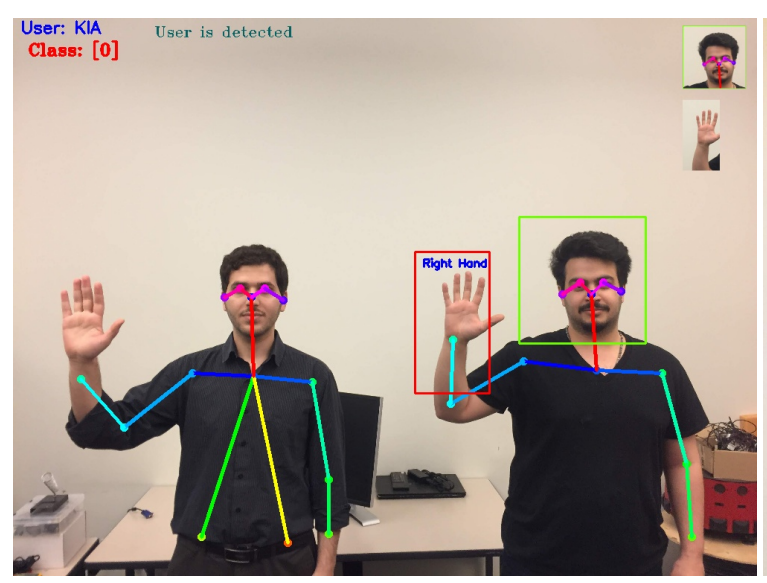

(a) Class \#1: take off

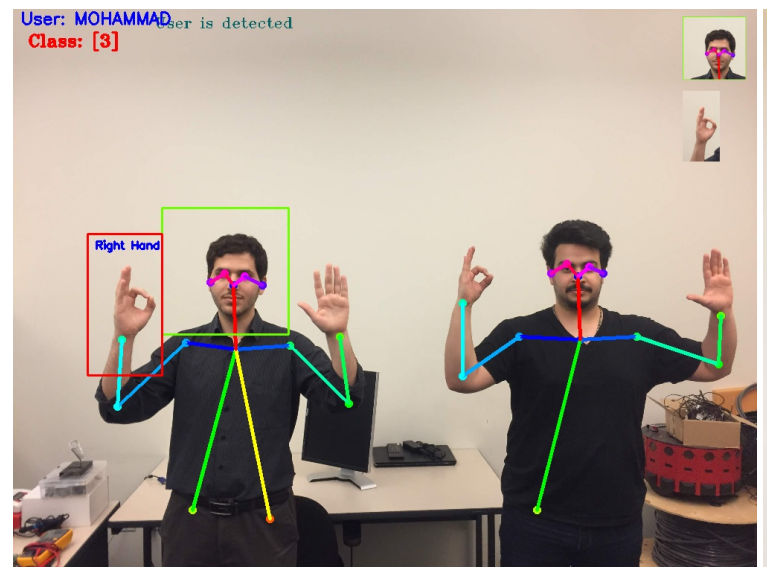

(c) Class \#4: turn right

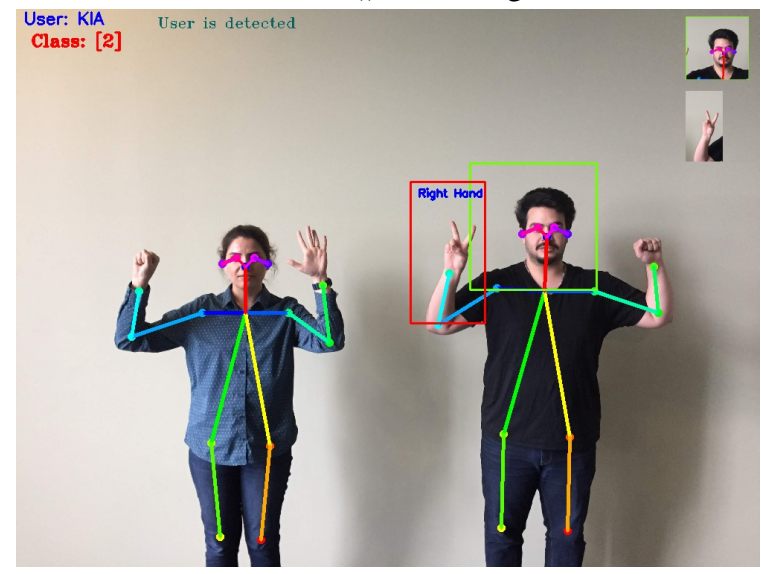

(e) Class \#3: turn left
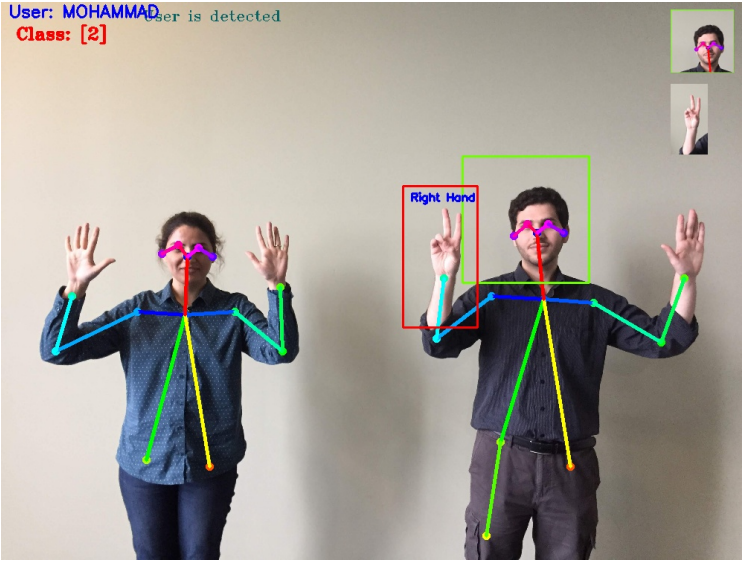

(b) Class \#3: turn left

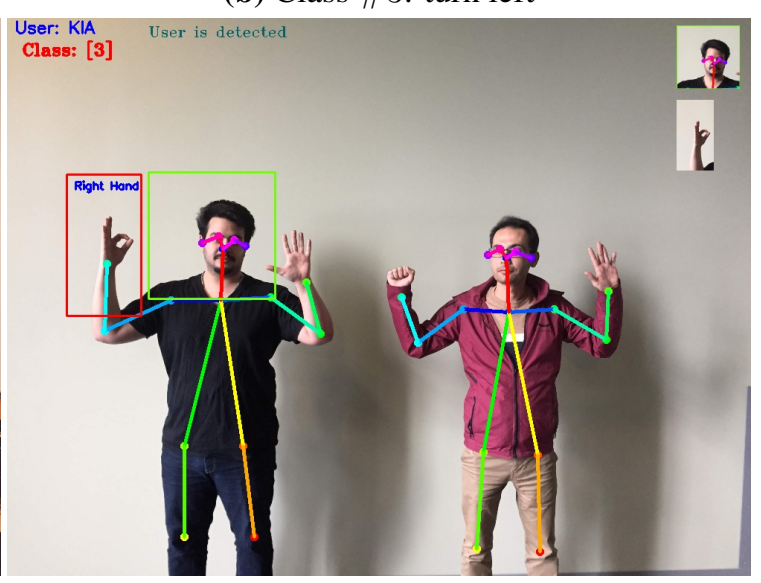

(d) Class \#4: turn right
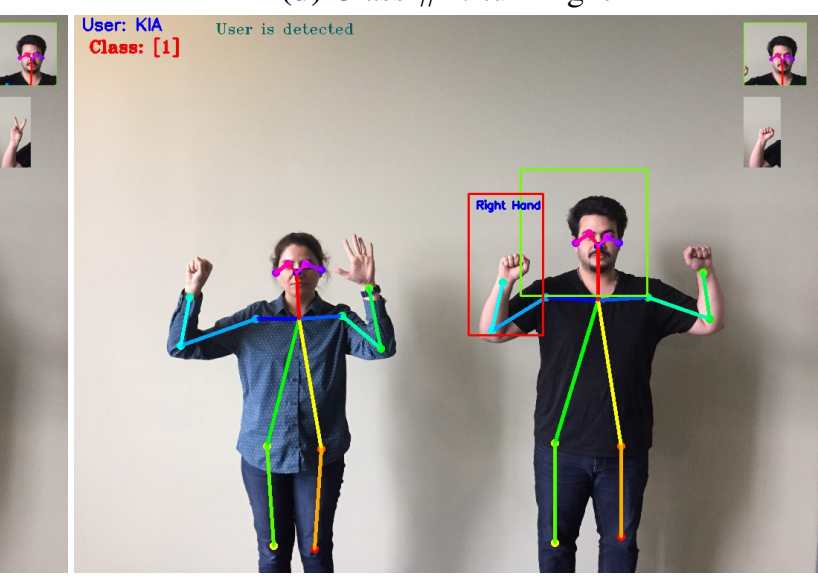

(f) Class \#2: land

Figure 5.18: Gesture recognition on two (2) subjects including a main subject gesturing in front of the camera (series 5). 


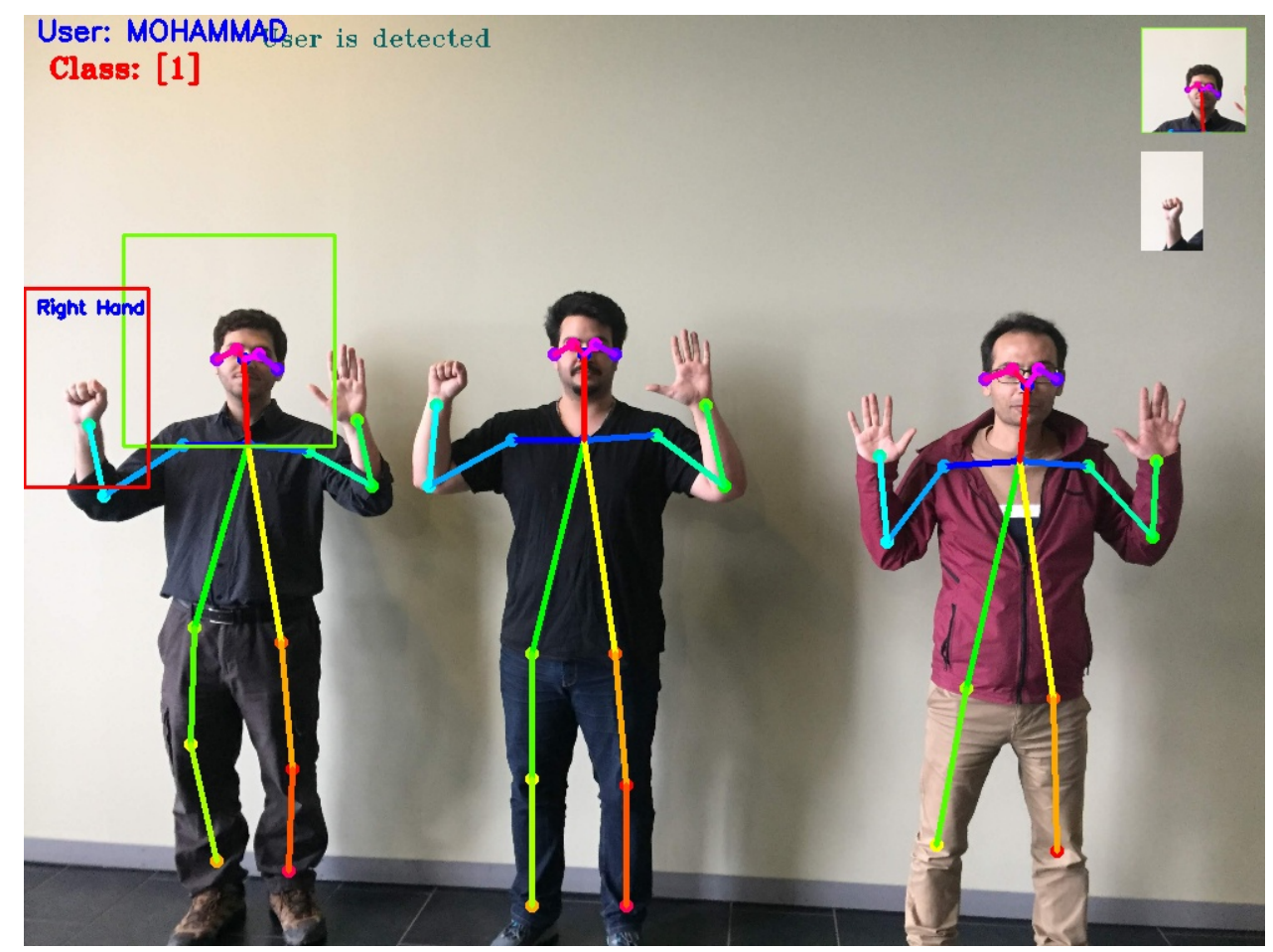

(a) Class \#2: land

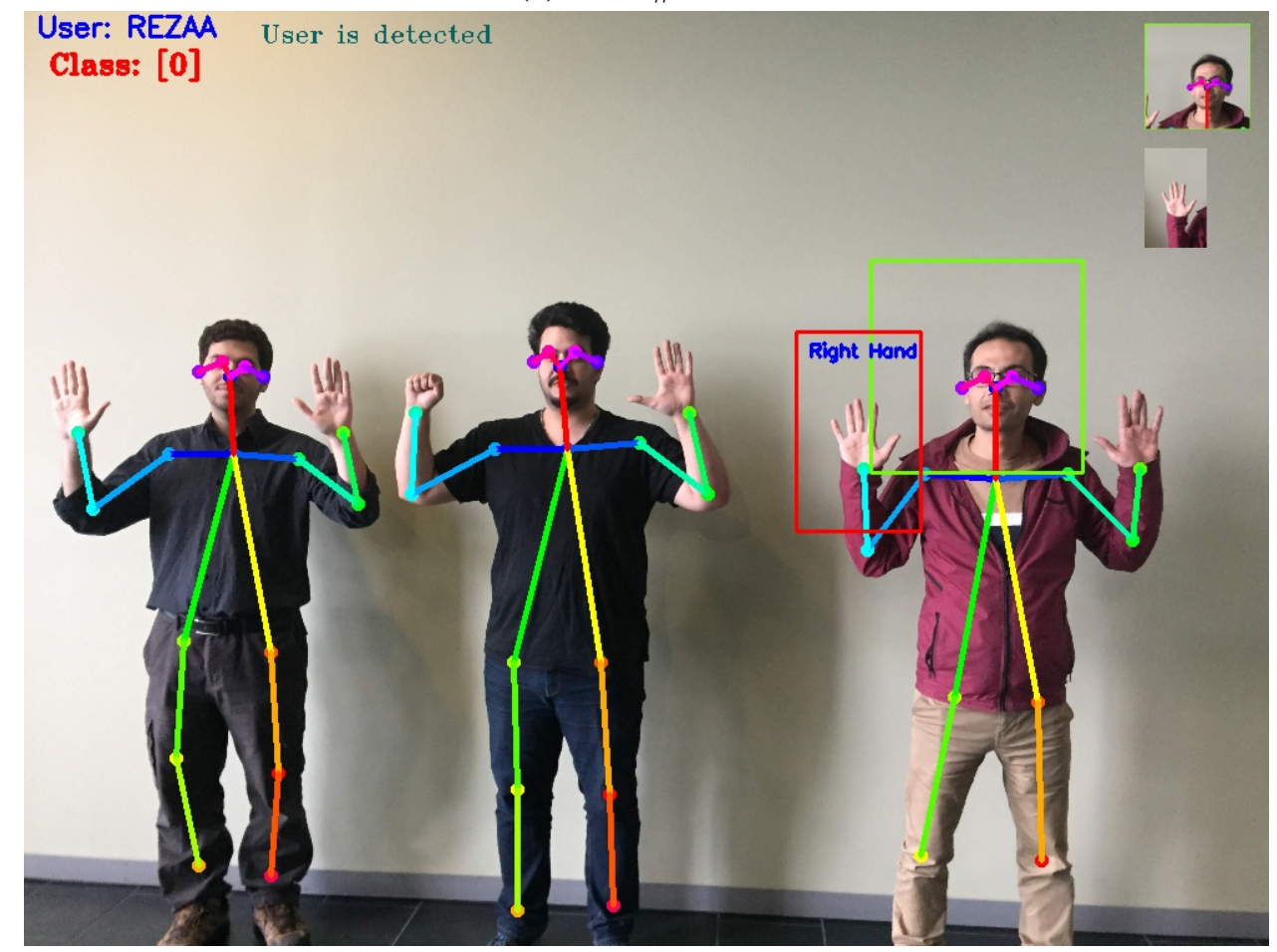

(b) Class \#1: take off

Figure 5.19: Gesture recognition on three (3) subjects including a main subject gesturing in front of the camera (series 1). 


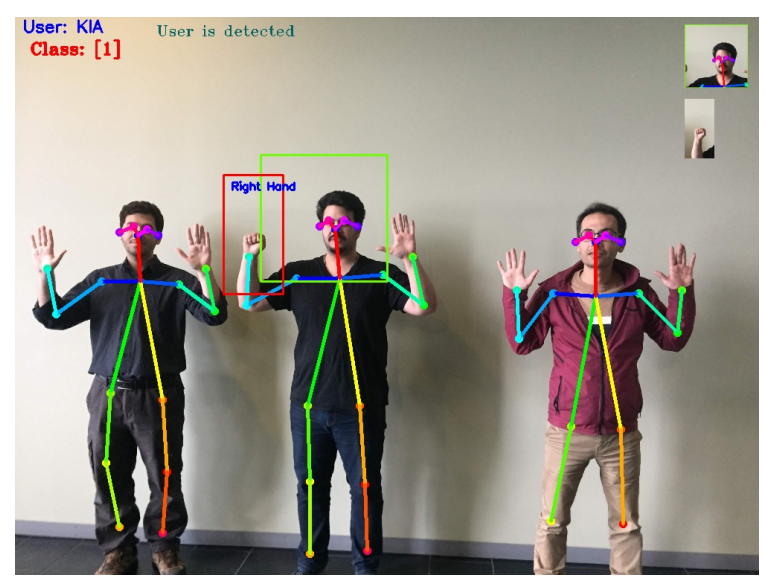

(a) Class \#2: land

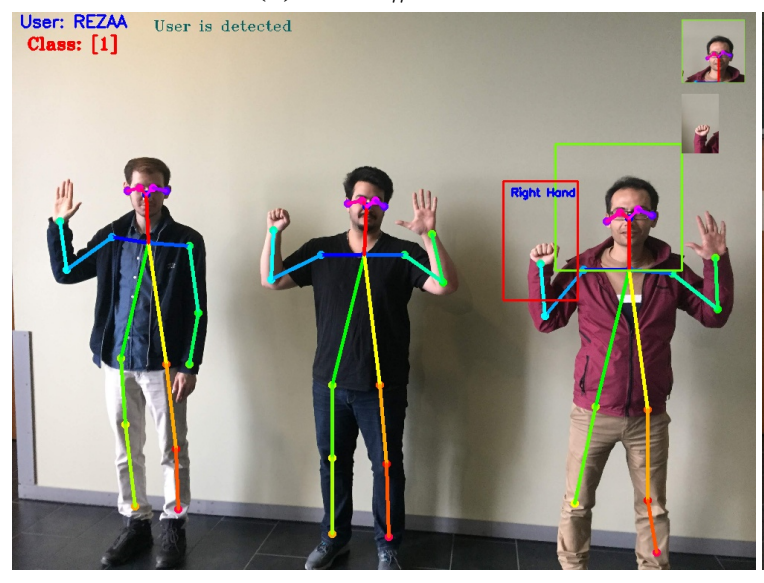

(c) Class \#2: land

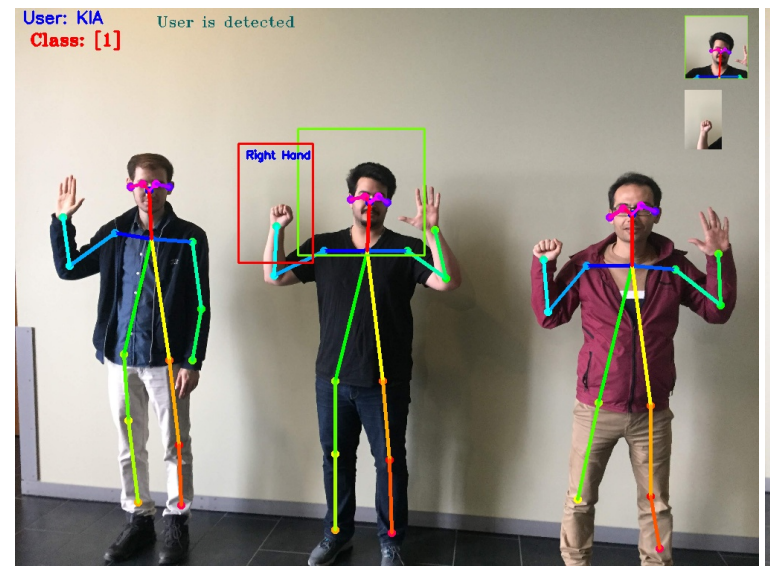

(e) Class \#2: land

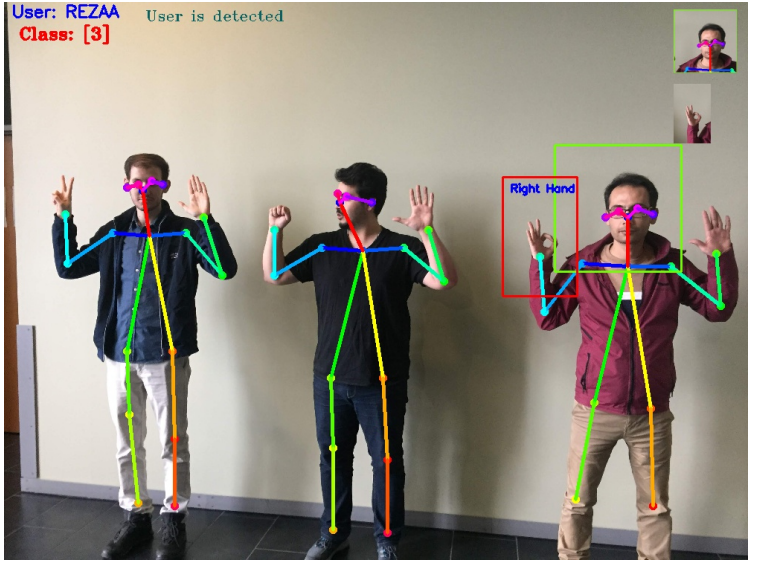

(b) Class \#4: turn right

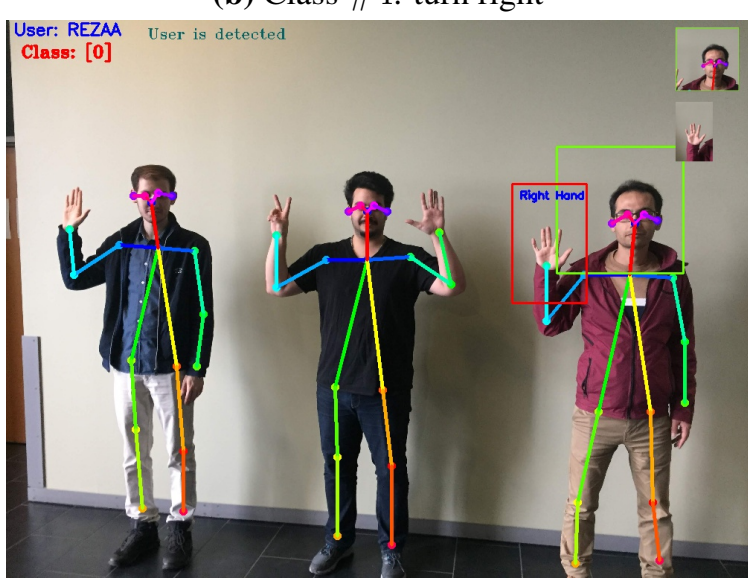

(d) Class \#1: take off

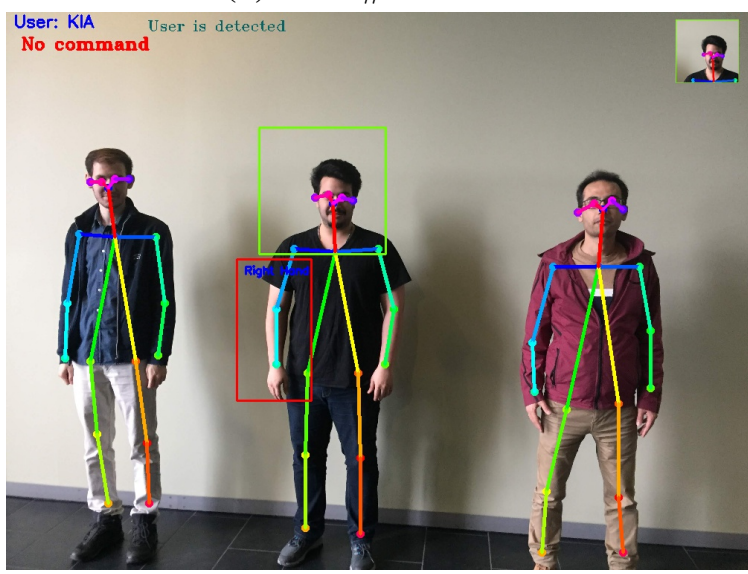

(f) No posture

Figure 5.20: Gesture recognition on three (3) subjects including a main subject gesturing in front of the camera (series 2). 


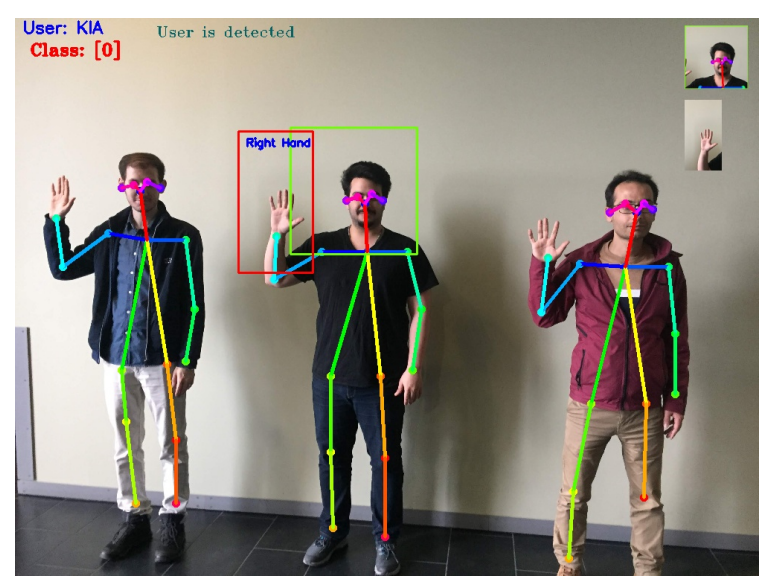

(a) Class \#1: take off

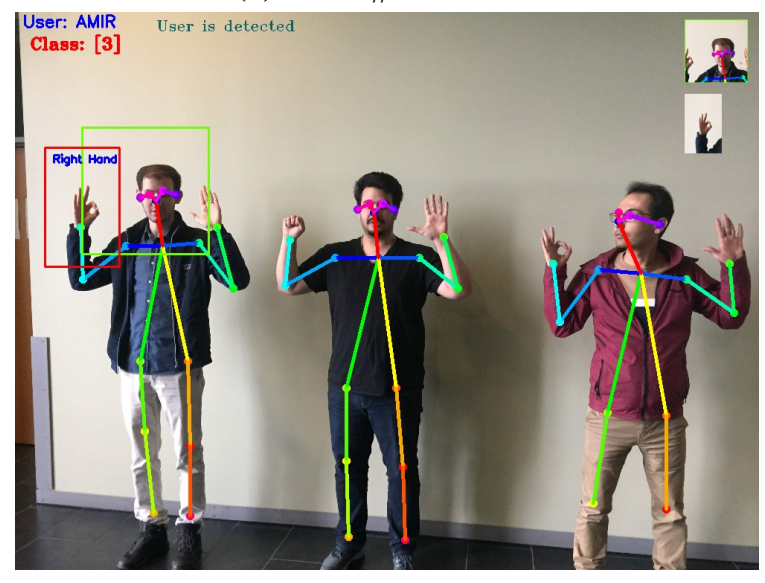

(c) Class \#4: turn right

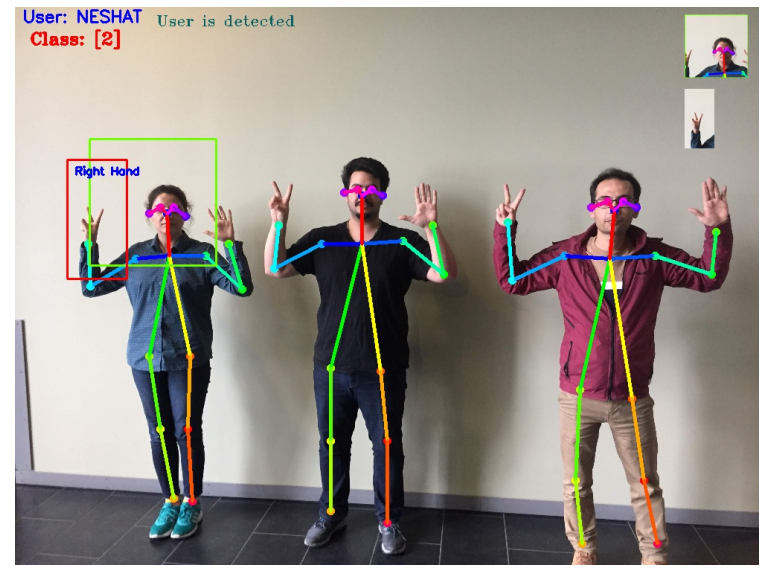

(e) Class \#3: turn left

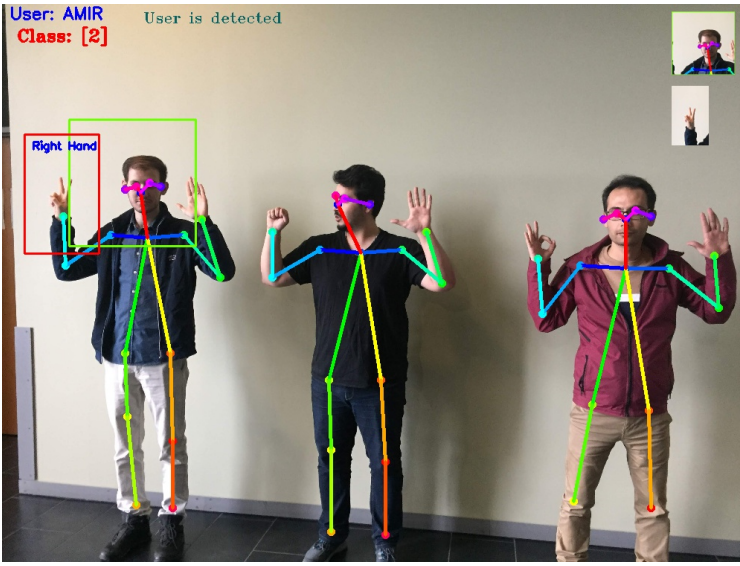

(b) Class \#3: turn left

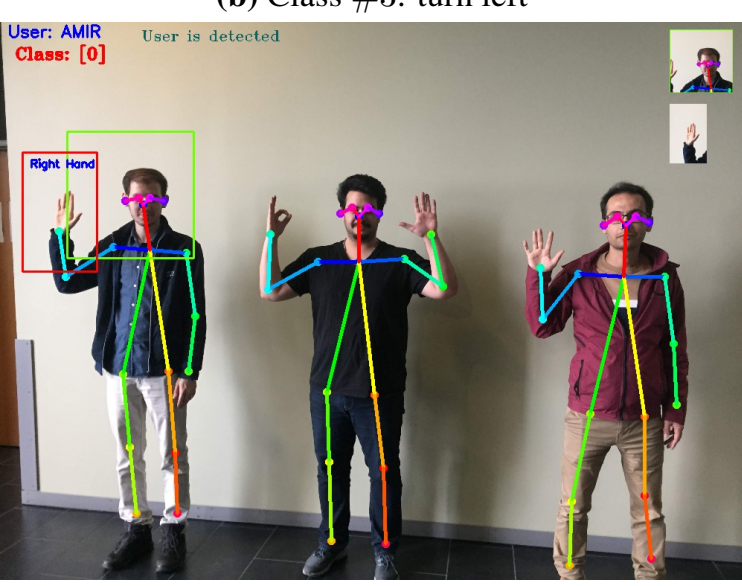

(d) Class \#1: take off

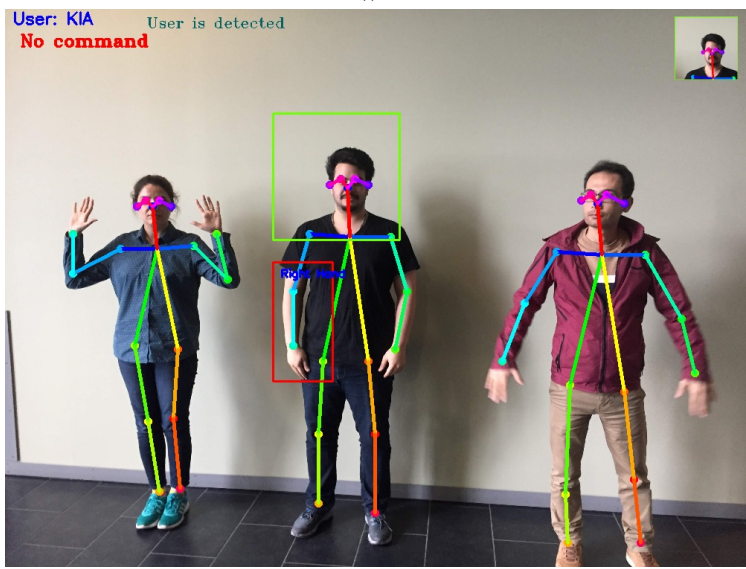

(f) No posture

Figure 5.21: Gesture recognition on three (3) subjects including a main subject gesturing in front of the camera (series 3). 


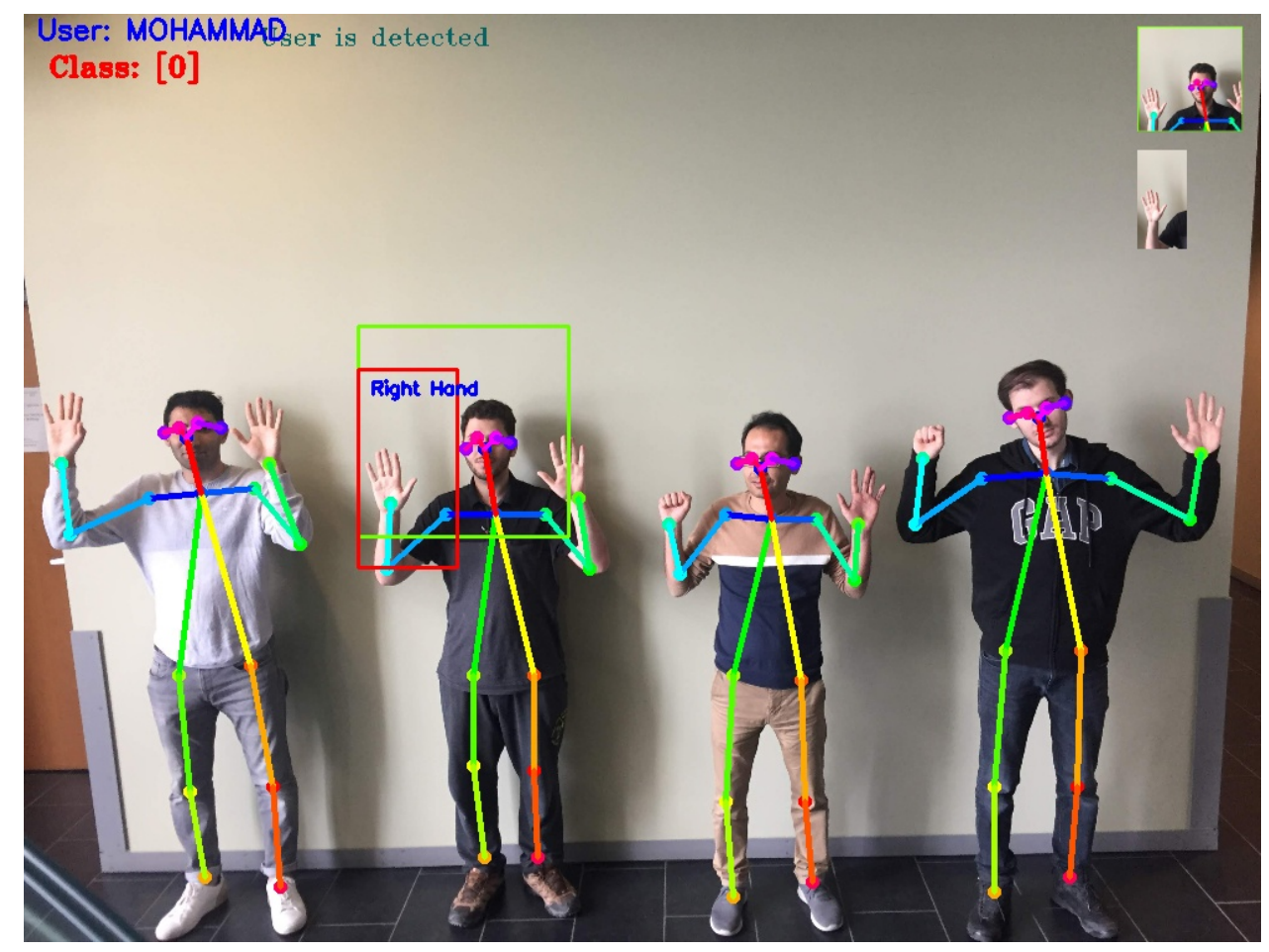

(a) Class \#1: take off

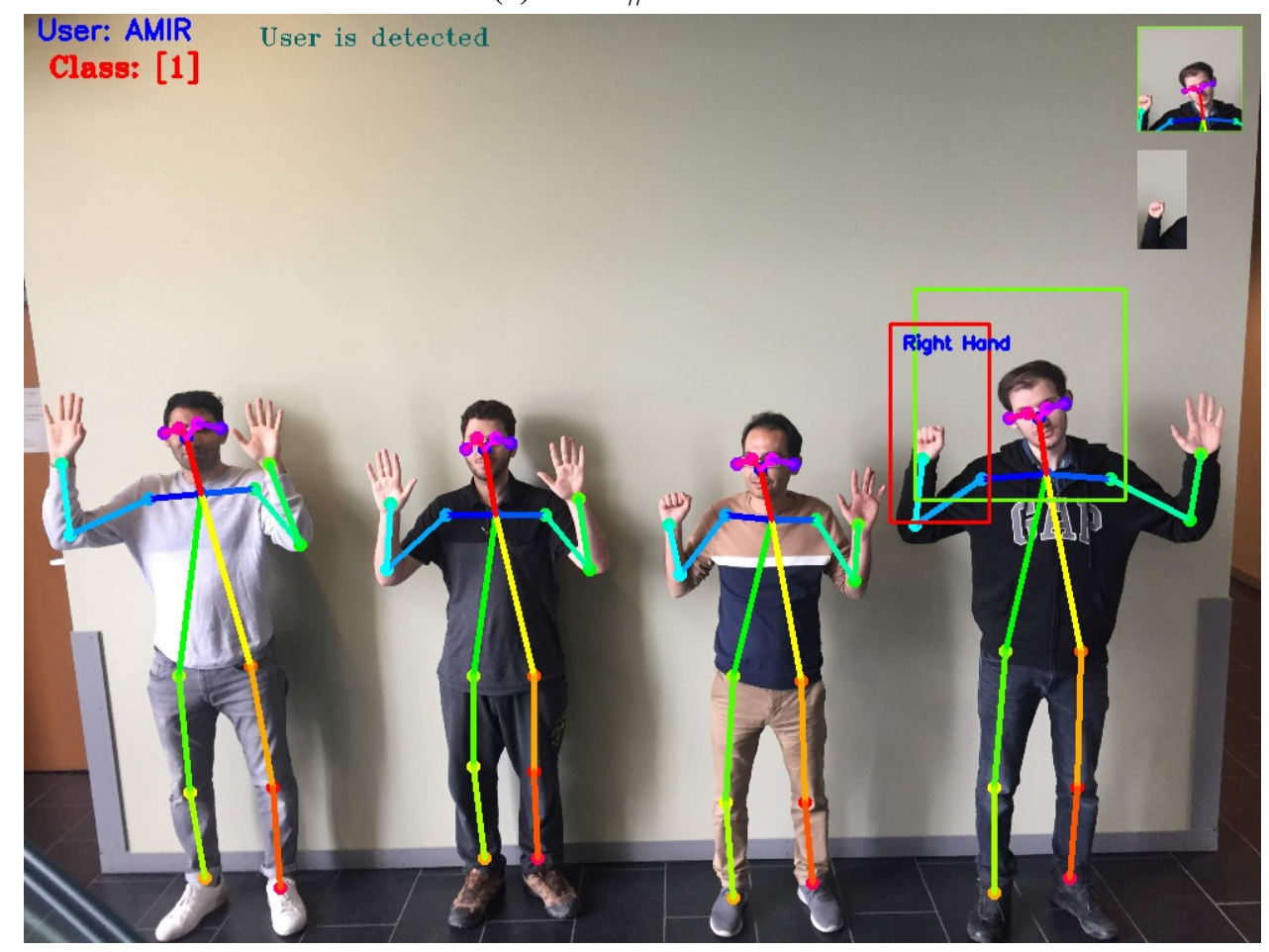

(b) Class \#2: land

Figure 5.22: Gesture recognition on group of four (4) subjects including a main subject performing in front of the camera (series 1). 


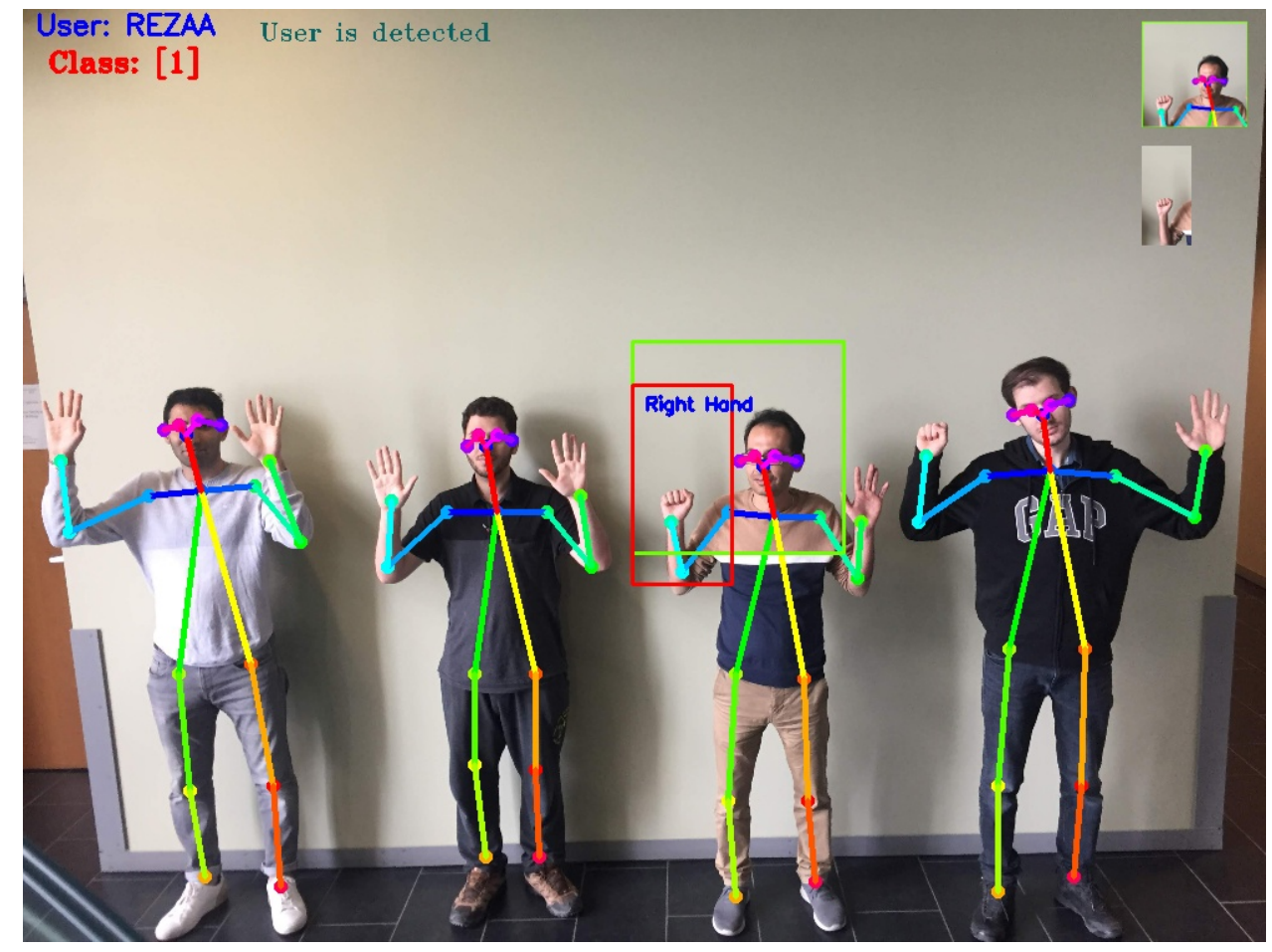

(a) Class \#2: land

Figure 5.23: Gesture recognition on group of four (4) subjects including a main subject performing in front of the camera (series 2). 


\section{Chapter 6}

\section{Conclusion and Future Work}

We presented a gesture-based control system for multi-rotor aerial vehicles, which is able to detect and classify both right-hand and left-hand gestures in single- and multisubject environments. The system is capable of operating with two different human-robot interaction subsystems: (i) a smart glove that produces continuous and discrete control commands and (ii) an image processing method that produces discrete commands using hand tracking and gesture recognition.

The proposed method has empowered with three separate modules for classifying the right-hand gesture in a single-subject environment: (i) a Hand Detection module which has been used to detect the hand RoI in the input frame and sent it to the classifier, (ii) a Gesture Classification module which is used to classify the detected hand in the received RoI from the hand detector module into one of the predefined classes, and (iii) a Retrainer module that retrains the running CNN model with the newly captured images and then obtains new weights to fit them to the running $\mathrm{CNN}$ model. The algorithm could distinguish between different scenarios when the user showed a posture correctly, hid his/her right hand, or held his/her right hand towards the ground.

The proposed method, also has four separate modules to classify the right-hand gesture in a multi-subject environment: (i) Skeleton Detection module that is capable of detecting all subjects present in the frame and sending their face regions to the facial recognition to detect the main subject, (ii) facial recognition module that captures one face image from 
the main subject, then uses that image to find the main subject among the received head regions from the Skeleton Detection module. The facial recognition module sends back the head ID of the main subject to the Skeleton Detection module to find the correct right-hand RoI. (iii) Gesture classifier which is used to classify the detected main subject right-hand RoI into one of the predefined classes, and (iv) Retrainer which retrains the running CNN model as mentioned in the single-subject environment.

To classify the left-hand gesture in both single- and multi-subject environments, a set of four flex sensors and an MPU sensor were implemented on a glove. Additionally, an SVM model has been deployed to classify the flex sensors output signals as a discrete command. After the SVM model classified the flex sensors outputs, the proposed algorithm used the exacts changes of the bending values and the MPU output signals as their continuous control command.

The proposed method had three separate validation modules to improve the system performances: (i) Gesture Classifier Validation that could retrain the model when the confidences were less than the threshold based on new data from a new user, (ii) multi-rotor aerial vehicles control validation that caused adjustments to the controller, and (iii) Humanin-the-Loop Validation that monitored the drone behavior.

Unlike the other algorithms and methods for posture detection and classification, the proposed method does not have limitations requiring a user to stand alone in front of the camera or to show just one hand to the camera. The proposed algorithm distinguished between subjects to find the main subject, then classified his/her right-hand gesture. The algorithm was studied on four different group populations of random subjects with various physical features.

The proposed control system is implemented for a multi-rotor aerial vehicle. However, it can be used for other controllable devices or any unmanned vehicles by redefining the gestures and some modifications for the targeted device. 


\subsection{Limitations}

One of the limitations of the proposed method is the distance between the subjects and the camera. The subjects must be in the camera's field of view; otherwise the Skeleton Detection module can not detect all the subjects. The second limitation of the proposed method is the separation between the subjects. Since we are using 2D images, the Skeleton Detection module can not distinguish the subjects if there is no separation between the subjects. The third limitation of the proposed method is the lighting of the environment. Since the Skeleton Detection accuracy and Gesture Classification accuracy are based on the input frame, the proposed method might not be able to detect all the subjects and their gestures in poor lighting conditions.

\subsection{Future Work}

This project has many applications namely, control an unman aerial vehicle (UAV) or an unman ground vehicle (UGV) from a far distance where we can set a control station and the user can control the targeted robot through from the control station. As a part of the future work we want to add more wearable sensors such as:

- An inertial measurement unit (IMU) sensor will be embedded in the smart glove to measure the hand's acceleration and rotation accurately.

- An electromyography (EMG) sensor will be used on the user's arm to gather electrical signals generated by the user's muscles.

We will use these newly gathered data to train an SVM model to classify additional gestures and create additional commands for control purposes. We will develop a mapping system that helps the user to integrate the proposed algorithm with any controllable robot.

Furthermore, we will scale and develop this project as follows:

- Develop a gesture-based control system that will detect multiple main subjects in a multi-subject environment and classify their right/left-hands gestures. We will apply this multi-user feature to a multi-section robot (a robot with multiple hands, for 
instance) where each user can control a specific part of the robot, and the robot performs all the commands simultaneously.

- Develop smart glasses that have an in-built camera and integrate it into the proposed system. These smart glasses enable the user to perform the gestures in front of his/her face. The smart glasses can connect to other users to share the data and accomplish a multi-user task. We need to retrain the $\mathrm{CNN}$ model with the gestures from the back of the right hand. Nevertheless, the SVM model (smart glove) does not need any new training, and it will be connected to the glasses to send the data to the targeted UAV or UGV. 


\section{Bibliography}

[1] K. K. Lekkala and V. K. Mittal, "Simultaneous aerial vehicle localization and human tracking," in 2016 IEEE Region 10 Conference (TENCON). IEEE, 2016, pp. 379383.

[2] K. K. Lekkala and V. K. Mittal, "Accurate and augmented navigation for quadcopter based on multi-sensor fusion," in 2016 IEEE Annual India Conference (INDICON). IEEE, 2016, pp. 1-6.

[3] A. G. Perera, Y. Wei Law, and J. Chahl, "Uav-gesture: A dataset for uav control and gesture recognition," in Proceedings of the European Conference on Computer Vision (ECCV), 2018.

[4] N. SaiChinmayi, C. Hasitha, B. Sravya, and V. Mittal, "Gesture signals processing for a silent spybot," in 2015 2nd International Conference on Signal Processing and Integrated Networks (SPIN). IEEE, 2015, pp. 756-761.

[5] Y. Ma, Y. Liu, R. Jin, X. Yuan, R. Sekha, S. Wilson, and R. Vaidyanathan, "Hand gesture recognition with convolutional neural networks for the multimodal uav control," in 2017 Workshop on Research, Education and Development of Unmanned Aerial Systems (RED-UAS). IEEE, 2017, pp. 198-203.

[6] S.-Y. Shin, Y.-W. Kang, and Y.-G. Kim, "Hand gesture-based wearable human-drone interface for intuitive movement control," in 2019 IEEE International Conference on Consumer Electronics (ICCE). IEEE, 2019, pp. 1-6. 
[7] A. Asokan, A. J. Pothen, and R. K. Vijayaraj, "Armatron-a wearable gesture recognition glove: For control of robotic devices in disaster management and human rehabilitation," in 2016 International Conference on Robotics and Automation for Humanitarian Applications (RAHA). IEEE, 2016, pp. 1-5.

[8] D. Bannach, O. Amft, K. S. Kunze, E. A. Heinz, G. Troster, and P. Lukowicz, "Waving real hand gestures recorded by wearable motion sensors to a virtual car and driver in a mixed-reality parking game," in 2007 IEEE Symposium on Computational Intelligence and Games. IEEE, 2007, pp. 32-39.

[9] M. Obaid, O. Mubin, C. A. Basedow, A. A. Ünlüer, M. J. Bergström, and M. Fjeld, “A drone agent to support a clean environment," in Proceedings of the 3rd International Conference on Human-Agent Interaction. ACM, 2015, pp. 55-61.

[10] T. Chouhan, A. Panse, A. K. Voona, and S. Sameer, "Smart glove with gesture recognition ability for the hearing and speech impaired," in 2014 IEEE Global Humanitarian Technology Conference-South Asia Satellite (GHTC-SAS). IEEE, 2014, pp. $105-110$.

[11] K. Natarajan, T.-H. D. Nguyen, and M. Mete, "Hand gesture controlled drones: An open source library," in Proceedings of the 1st International IEEE Conference on Data Intelligence and Security (ICDIS), Apr. 2018, pp. 168-175.

[12] W. Liu, D. Anguelov, D. Erhan, C. Szegedy, S. Reed, C.-Y. Fu, and A. C. Berg, "Ssd: Single shot multibox detector," in Proceedings of the European Conference on Computer Vision (ECCV). Springer, 2016, pp. 21-37.

[13] K. Haratiannejadi, N. E. Fard, and R. R. Selmic, "Smart glove and hand gesturebased control interface for multi-rotor aerial vehicles," in 2019 IEEE International Conference on Systems, Man and Cybernetics (SMC), 2019, pp. 1956-1962. 
[14] J. Hosang, R. Benenson, and B. Schiele, "Learning non-maximum suppression," in Proceedings of the IEEE Conference on Computer Vision and Pattern Recognition, Jul. 2017, pp. 4507-4515.

[15] P. Zhu, J. Isaacs, B. Fu, and S. Ferrari, "Deep learning feature extraction for target recognition and classification in underwater sonar images," in Proceedings of the IEEE 56th Annual Conference on Decision and Control (CDC), Dec. 2017, pp. 27242731.

[16] D. Yu, H. Wang, P. Chen, and Z. Wei, "Mixed pooling for convolutional neural networks," in International conference on rough sets and knowledge technology. Springer, 2014, pp. 364-375.

[17] N. Srivastava, G. Hinton, A. Krizhevsky, I. Sutskever, and R. Salakhutdinov, "Dropout: A simple way to prevent neural networks from overfitting," The Journal of Machine Learning Research, vol. 15, no. 1, pp. 1929-1958, Jun. 2014.

[18] S. Cai, Y. Shu, W. Wang, M. Zhang, G. Chen, and B. C. Ooi, "Effective and efficient dropout for deep convolutional neural networks," arXiv preprint arXiv:1904.03392, 2019.

[19] B. Karlik and A. V. Olgac, "Performance analysis of various activation functions in generalized mlp architectures of neural networks," International Journal of Artificial Intelligence and Expert Systems, vol. 1, no. 4, pp. 111-122, 2011.

[20] Z. Zhang, "Improved adam optimizer for deep neural networks," in 2018 IEEE/ACM 26th International Symposium on Quality of Service (IWQoS). IEEE, 2018, pp. 1-2.

[21] E. Dogo, O. Afolabi, N. Nwulu, B. Twala, and C. Aigbavboa, "A comparative analysis of gradient descent-based optimization algorithms on convolutional neural networks," in 2018 International Conference on Computational Techniques, Electronics and Mechanical Systems (CTEMS). IEEE, 2018, pp. 92-99. 
[22] D. P. Kingma and J. Ba, "Adam: A method for stochastic optimization," arXiv preprint arXiv:1412.6980, 2014.

[23] R. Girshick, J. Donahue, T. Darrell, and J. Malik, "Region-based convolutional networks for accurate object detection and segmentation," IEEE transactions on pattern analysis and machine intelligence, vol. 38, no. 1, pp. 142-158, 2015.

[24] A. J. Smola and B. Schölkopf, “A tutorial on support vector regression,” Statistics and computing, vol. 14, no. 3, pp. 199-222, 2004.

[25] C. J. Burges, “A tutorial on support vector machines for pattern recognition," Data mining and knowledge discovery, vol. 2, no. 2, pp. 121-167, 1998.

[26] J. Zhang and L. Chen, "Clustering-based undersampling with random over sampling examples and support vector machine for imbalanced classification of breast cancer diagnosis," Computer Assisted Surgery, vol. 24, no. sup2, pp. 62-72, 2019.

[27] Z. Zhang, "Microsoft kinect sensor and its effect," IEEE Multimedia, vol. 19, no. 2, pp. 4-10, 2012.

[28] A. Sanna, F. Lamberti, G. Paravati, and F. Manuri, "A kinect-based natural interface for quadrotor control," Entertainment Computing, vol. 4, no. 3, pp. 179-186, 2013.

[29] G. A. Ten Holt, M. J. Reinders, and E. Hendriks, "Multi-dimensional dynamic time warping for gesture recognition," in Proceedings of the Thirteenth Annual Conference of the Advanced School for Computing and Imaging, 2007, pp. 23-32.

[30] J. Triesch and C. Von Der Malsburg, "A system for person-independent hand posture recognition against complex backgrounds," IEEE Transactions on Pattern Analysis and Machine Intelligence, vol. 23, no. 12, pp. 1449-1453, 2001.

[31] S. Bodiroža, H. I. Stern, and Y. Edan, "Dynamic gesture vocabulary design for intuitive human-robot dialog," in Proceedings of the seventh annual ACM/IEEE International Conference on Human-Robot Interaction, Mar. 2012, pp. 111-112. 
[32] S. Bodiroža, G. Doisy, and V. V. Hafner, "Position-invariant, real-time gesture recognition based on dynamic time warping," in Proceedings of the 8th ACM/IEEE International Conference on Human-Robot Tnteraction, Mar. 2013, pp. 87-88.

[33] J. Shotton, A. Fitzgibbon, M. Cook, T. Sharp, M. Finocchio, R. Moore, A. Kipman, and A. Blake, "Real-time human pose recognition in parts from single depth images," in Proceedings of the IEEE Conference on Computer Vision and Pattern Recognition (CVPR), 2011, pp. 1297-1304.

[34] T. Simon, H. Joo, I. Matthews, and Y. Sheikh, "Hand keypoint detection in single images using multiview bootstrapping," in Proceedings of the IEEE Conference on Computer Vision and Pattern Recognition, 2017, pp. 1145-1153.

[35] A. Corradini, "Dynamic time warping for off-line recognition of a small gesture vocabulary," in Proceedings of the IEEE ICCV Workshop on Recognition, Analysis, and Tracking of Faces and Gestures in Real-Time Systems, Jul. 2001, pp. 82-89.

[36] G. Doisy, A. Jevtić, and S. Bodiroža, "Spatially unconstrained, gesture-based humanrobot interaction," in Proceedings of the 8th ACM/IEEE International Conference on Human-Robot Interaction, Mar. 2013, pp. 117-118.

[37] R. Girshick, J. Donahue, T. Darrell, and J. Malik, "Region-based convolutional networks for accurate object detection and segmentation," IEEE Transactions on Pattern Analysis and Machine Intelligence, vol. 38, no. 1, pp. 142-158, Jan. 2016.

[38] K. A. Bhaskaran, A. G. Nair, K. D. Ram, K. Ananthanarayanan, and H. N. Vardhan, "Smart gloves for hand gesture recognition: Sign language to speech conversion system," in Robotics and Automation for Humanitarian Applications (RAHA), 2016 International Conference on. IEEE, 2016, pp. 1-6.

[39] J. Bae, A. Larson, R. M. Voyles, R. Godzdanker, and J. Pearce, "Development and user testing of the gestural joystick for gloves-on hazardous environments," in Robot 
and Human interactive Communication, 2007. RO-MAN 2007. The 16th IEEE International Symposium on. IEEE, 2007, pp. 1096-1101.

[40] A. Géron, Hands-On Machine Learning with Scikit-Learn, Keras, and TensorFlow: Concepts, Tools, and Techniques to Build Intelligent Systems. O'Reilly Media, 2019.

[41] Z. Cao, G. Hidalgo, T. Simon, S.-E. Wei, and Y. Sheikh, "Openpose: realtime multiperson $2 \mathrm{~d}$ pose estimation using part affinity fields," arXiv preprint arXiv:1812.08008, 2018.

[42] S. Qiao, Y. Wang, and J. Li, "Real-time human gesture grading based on openpose," in 2017 10th International Congress on Image and Signal Processing, BioMedical Engineering and Informatics (CISP-BMEI). IEEE, 2017, pp. 1-6.

[43] P. J. Thilaga, B. A. Khan, A. Jones, and N. K. Kumar, "Modern face recognition with deep learning," in 2018 Second International Conference on Inventive Communication and Computational Technologies (ICICCT). IEEE, 2018, pp. 1947-1951.

[44] O. Déniz, G. Bueno, J. Salido, and F. De la Torre, "Face recognition using histograms of oriented gradients," Pattern Recognition Letters, vol. 32, no. 12, pp. 1598-1603, 2011.

[45] A. Bulat and G. Tzimiropoulos, "How far are we from solving the $2 \mathrm{~d} \& 3 \mathrm{~d}$ face alignment problem? (and a dataset of 230,000 3d facial landmarks)," in International Conference on Computer Vision, 2017. 